.

Francesco Bimbo

Adding value through health claims: an empirical analysis of the Italian yogurt market

7
0
0
0
0
0
0
0
0
0

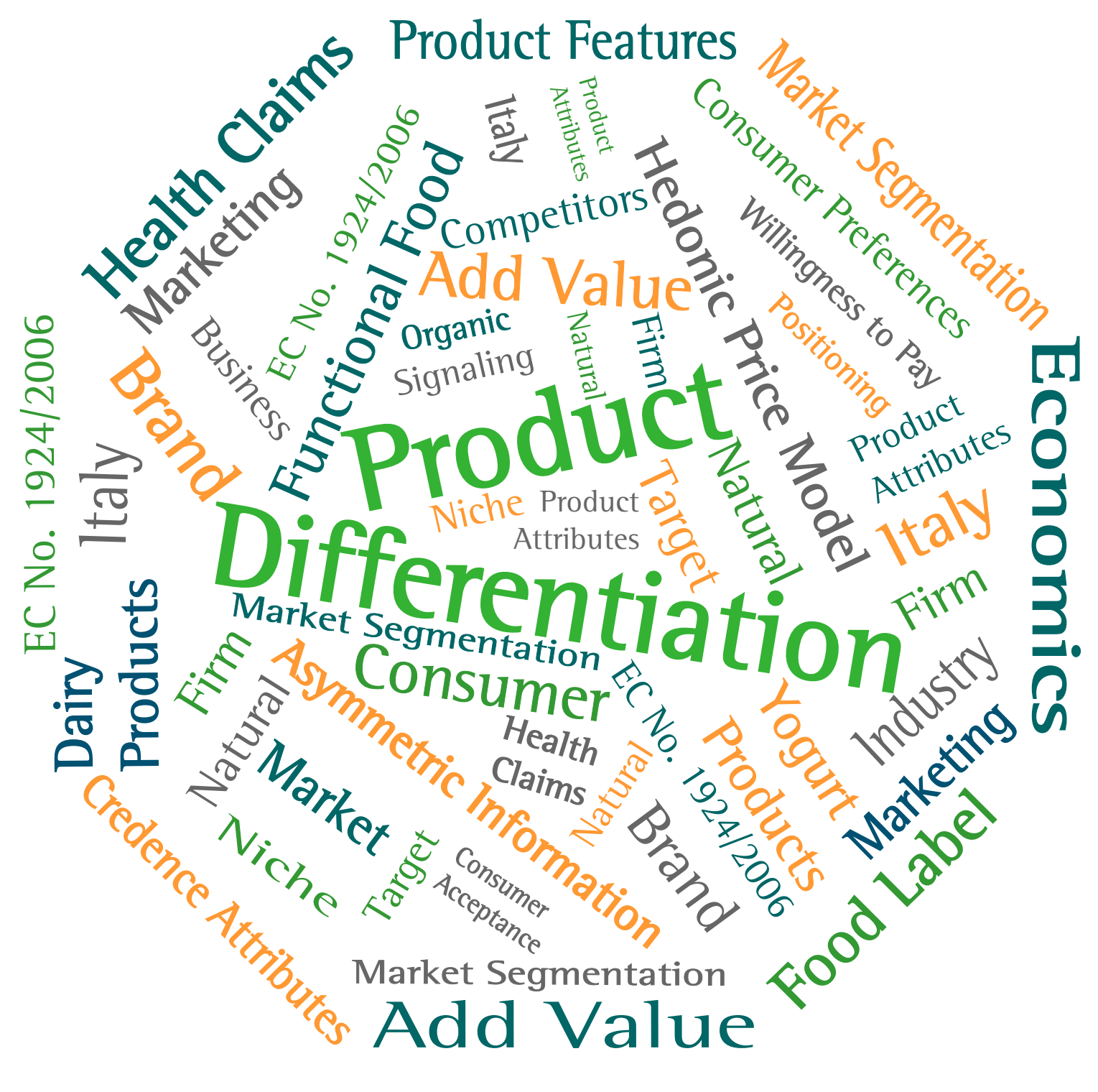




\section{Propositions}

1. "Risk diseases reduction" claims have higher implicit prices than "general function" claims.

(this thesis)

2. Natural and organic claims have implicit prices similar in magnitude to health claims supporting bone health and the immune system.

(this thesis)

3. Neoclassical economic theory often fails in explaining the real world by assuming that economic agents behave rationally.

4. Technological advancements in automation and robotics that destroy millions of jobs require coherent government policies to limit negative social consequences.

5. A PhD program that teaches persistence pays off.

6. High quality data and up-to-date econometric tools are worthless if applied to the wrong research question.

Propositions belonging to the thesis, entitled

"Adding value through health claims: an empirical analysis of the Italian yogurt market".

Francesco Bimbo

Wageningen, 5 September 2017 


\title{
Adding value through health claims: \\ an empirical analysis of the
}

\author{
Italian yogurt market
}

Francesco Bimbo 


\section{Thesis committee}

\section{Promotor}

Prof. Dr A.G.J.M. Oude Lansink

Professor of Business Economics

Wageningen University \& Research

\section{Co-promotor}

Dr Alessandro Bonanno

Associate Professor of Agricultural and Resource Economics

Colorado State University, USA

\section{Other members}

Prof. Dr Wim Verbeke, Ghent University, Belgium

Prof. Dr S.W.F. Onno Omta, Wageningen University \& Research

Dr Liesbeth K.E. Dries, Wageningen University \& Research

Prof. Alessandro Banterle, University of Milan, Italy

This research was conducted under the auspices of the Wageningen School of Social Sciences (WASS). 


\title{
Adding value through health claims: \\ an empirical analysis of the \\ Italian yogurt market
}

\author{
Francesco Bimbo
}

Thesis

submitted in fulfilment of the requirement for the degree of doctor at Wageningen University

by the authority of the Rector Magnificus,

Prof. Dr A.P.J. Mol,

in the presence of the

Thesis Committee appointed by the Academic Board

to be defended in public

on Tuesday 5 September 2017

at 11 a.m. in the Aula. 


\section{F (Francesco) Bimbo}

Adding value through health claims: an empirical analysis of the Italian yogurt market

138 pages.

Cover design: Arch. Roberto Potenza

$\mathrm{PhD}$ thesis, Wageningen University, Wageningen, the Netherlands (2017)

With references, with summary in English

ISBN: 978-94-6343-450-8

DOI: https://doi.org/10.18174/416380 


\section{Table of Contents}

$\begin{array}{ll}\text { Abstract } & 1\end{array}$

Chapter 1 General Introduction 4

Chapter 2 Consumers' acceptance and preferences for nutrition-modified and 16 functional dairy products: a systematic review

Chapter 3 Do Health Claims' Add Value? The Role of Functionality, 50 Effectiveness, and Brand

Chapter 4 Credence Attributes and the Quest for a Higher Price - a Hedonic Stochastic Frontier Approach

Chapter 5 Body Image Dissatisfaction and Health-Enhancing Food Choices An Analysis of Italian Yogurt Consumers

Chapter 6 General Discussion 


\begin{abstract}
In recent years policymakers have aimed to increase the general awareness regarding the link between diet and health, providing food companies with an opportunity to add healthenhancing features in order to differentiate their products. The European market of these products, referred to here as functional foods, is regulated according to the European regulation 1924/2006 on nutrition and health claims on foods.
\end{abstract}

Even though the European functional foods' market has grown steadily over the last 20 years, more than 3 out of 4 new functional products are withdrawn from the market within two years from launch. Indeed, firms have to overcome three main obstacles to market health-enhancing products successfully. First, consumer-related factors, such as the heterogeneity of consumer acceptance of, and preferences for, new functional products; second, firms' difficulties in selecting the optimal attribute profiles to meet consumer demand for health features and; third, firms' difficulties to target consumers with a high enough willingness to pay to ensure the profitability of those investments needed to develop and market functional products.

The general objective of this thesis is to assess quantitatively the implicit price of health claims as well as to generate new knowledge that will inform firms on how to target consumers with higher WTP for functional products.

This thesis uses the Italian yogurt market as a case study for three reasons. First, yogurt is considered intrinsically healthy and it is one of the most credible carriers of functional ingredients: the category of fermented functional dairy accounts for nearly 43 per cent of the total functional products' market. Second, among European countries, Italy saw the launch of the highest number (592) of new healthy products in the period 2005-2009; the majority of the new products introduced were functional yogurts benefiting digestive health (336), the immune system (99) and cardiovascular health (65). Third, the Italian yogurt market is characterized by a high level of differentiation with respect of health attributes, with manufacturers offering a variety of products carrying health claims, appearing suitable to analyze the problem at hand.

The results of this thesis show that in the Italian yogurt market the implicit prices of "risk reduction" claim is higher than that of "general function" ones (respectively, $+222.6 \%$ vs $+85.5 \%$ of the baseline alternative's price in the case of cholesterol reduction health claim), and in three out of four cases, health claims supported by familiar brands have higher implicit price than those supported by less-familiar ones. Those results confirm findings collected in the systematic literature review which highlights a consensus that claims supported by familiar brands as well as "risk reduction" (vis-à-vis "general function") ones increase consumers' acceptance of functional dairy products. The results also point out that "natural" and "organic" claims lead to implicit prices which are (approximately) $+46.6 \%$ and $+102.0 \%$ over the price of the baseline alternative, comparable to those of some of the health claims. Other results indicate that manufacturers operating in the Italian yogurt market investing in credence attributes (health claims, organic and natural) gain the ability to target consumers with higher WTP by including two or more products carrying credence attribute in their 
portfolio. Lastly, the findings of this thesis reveal that consumers' body image dissatisfaction, along with not reading food labels and lower knowledge about leading functional yogurt brands, are associated with lower number of functional yogurt packages purchased by Italian yogurt consumers using a virtual shelf setting.

Keywords: functional food, health claims, credence attributes. 
Chapter 1

General Introduction 


\subsection{Background}

The rising incidence of non-communicable diseases (such as heart stroke, high blood pressure, diabetes, coronary heart disease, and arthritis) among the population is one of the most worrisome public health issues of the twenty-first century as it generates considerable individual and social costs (WHO, 2010; Dee et al., 2014).

As a result, European policymakers have aimed to increase the general awareness regarding the link between diet and health by promoting informed consumer choices (Traill, 2012) which in turn provides an incentive for food companies to pursue product differentiation strategies by adding health-enhancing features to their products (Réquillart and Soler, 2014). These novel food versions, referred to here as functional foods ${ }^{1}$, are mainly targeted to health oriented consumers willing to improve their health through their diet (Devcich et al., 2007).

The health benefits resulting from the consumption of functional products are not quantifiable by consumers at any stage of the purchasing process. Thus, a product's health-related features are de facto credence attributes (Darby and Karni, 1973; Grunert, 2005), since their presence cannot be verified by consumers even after repeated consumption. In markets characterized by the presence of products with credence attributes, asymmetric information may lead to welfare losses (Caswell and Padberg, 1992; Caswell and Mojduszka, 1996; Teisl and Roe, 1998; Roe and Sheldon, 2007).

Prior to 2006, each European member state regulated its national market of functional foods, allowing food companies to use health claims with the only requirement that health claims had not to mislead consumers (Bech-Larsen and Scholderer, 2007). In 2006, the European Union created Regulation (EC) No.1924/2006 on nutrition and health claims on foods. The regulation became effective in 2007 through a set of uniform criteria among European member states to regulate the use of health claims. The three main aims of the regulation were to: 1) reduce asymmetric information between manufacturers and consumers; 2) protect consumers from untruthful claims; and 3) promote and protect innovation in the EU food sector (Reg. (EC) No.1924/2006).

The European Regulation (EC) No.1924/2006 classifies health claims in two different groups: "Reduction of disease risk" (article 14) and "General function" claims (article 13). Even though both types of claims need to be approved by the European Food Safety Authority (EFSA), the approval protocols for the two different types of claims are different. The authorization procedure for "Reduction of disease risk" claims is based on a case-by-case dossier review process by the EFSA's panel on Dietetic Products, Nutrition and Allergies (NDA). Each dossier must provide scientific evidence supporting the claim and a causal relationship between the active principle and its claimed effect on health. Instead, the use of

\footnotetext{
${ }^{1}$ For an overview of the many definitions of functional food see Bigliardi and Galati's (2013) review.
} 
"General function" claims on a product's label is based upon existing knowledge or links between the active principle and health. ${ }^{2}$

In spite of the changes in regulatory schemes, in the last two decades food companies have intensified their efforts to develop and market new products with health-enhancing properties (Research and Markets, 2014). Data show that between 2008 and 2009 Europe witnessed the launch of a large number of health-enhancing products, and that Italy was the European market leader in product launches (325) followed by UK (237), Germany (235), and France (150) (Nutraingredients-US, 2009). Additionally, a recent report estimates that the global market for foods with health-enhancing features amounted to (approximately) $\$ 168$ billion in 2013 , with an annual average growth rate of $8.5 \%$, forecasted to exceed $\$ 300$ billion by 2020 (Research and Markets, 2014).

Thus, functional foods seem to satisfy the growing consumer need for health and nutrition (Wong et al., 2015) while at the same time generating an opportunity for food companies to engage in product differentiation and escape from price competition (Bimbo et al., 2016).

\subsection{Problem statement}

In spite of the growing interest regarding functional food products, research shows that over 75 per cent of new functional products launched in the European market are withdrawn within the first two years from launch (Stein and Rodriguez-Cerezo, 2008).

Such a high rate of product failure, which has a direct impact on firms' competitiveness, may be caused by three series of factors. First, consumer-related factors, such as the heterogeneity of consumer acceptance of, and preferences for, new functional products (Grunert, 2005; Bech-Larsen and Scholderer, 2007); second, firms' difficulties in selecting the optimal product attribute profiles to meet consumer demand for health features and; third, firms' difficulties to target consumers with a high enough willingness to pay to ensure the profitability of those investments needed to develop and market functional products (Stein and Rodriguez-Cerezo, 2008).

In fact, even though consumer acceptance of functional foods has been indicated as a key market success factor of new functional products (Verbeke, 2005), in many cases food companies developed new functional products in their R\&D departments, based on technical interest or feasibility (Bleiel, 2010) disregarding consumers' acceptance and preferences (Van Kleef et al., 2002; 2005b).

A considerable body of research exists that addresses consumers' acceptance of functional foods, finding that acceptance is higher for females and it increases with age, household size, nutrition knowledge, and product familiarity (van Kleef et al., 2005a; Ares and Gambaro,

\footnotetext{
${ }^{2}$ Article 13 claims are further divided in two categories: (i) Article 13.1: 'general function' claims related to growth, development and functions of the body; (ii) Article 13.5 claims pertaining to general function claims based on new and/or proprietary data. Article 13.5 and Article 14 claims undergo the same authorization procedure, illustrated in the main text. Regulation (EC) No. 1924/2006 also includes nutrition claims (Article 8), which are not considered in this analysis.
} 
2007; Ares et al., 2009; Ares et al. 2010; Annunziata and Vecchio, 2013). Furthermore, consumers' psychological factors contribute in shaping the acceptance of functional foods. Interest in health, the relevance of a specific functional product for one's health status (Labrecque et al., 2006; Urala and Lähteenmäki, 2004; 2007), and the perceived self-efficacy, are found to be good predictors of the individual consumption of functional food products (Cox, Evans and Lease, 2007). Instead, food neophobia may reduce the use of some types of functional options (Urala and Lähteenmäki, 2007; Siegrist et al., 2008).

Although consumer acceptance of, and preferences for, many functional food products have been explored at length in the literature (inter alia, Van Kleff et al., 2005a; Ares and Gambaro, 2007; Siegrist et al., 2008; Ares et al., 2009; Krutulyte et al., 2011) findings are often fragmented, conflicting, and difficult to reconcile (Van Kleef et al., 2005b). As a consequence, firms wanting to increase the rate of market success for new functional products have to first understand consumer choices for such products. Thus, gathering and comparing the multiple findings available in the literature is a necessary step for food manufacturers to refine their new functional product development strategies and to reduce the risk of market failure. Further, the large heterogeneity of results in the literature is mainly due to the fact that studies have been conducted in different countries using different scales assessing consumer acceptance and preferences ${ }^{3}$. Such studies have been based generally on stated preferences data, where small samples of respondents were asked to state their attitudes, perceptions and intentions toward a product concept using Likert scales or other ad hoc metrics.

These types of consumer behavior studies, using small samples and stated preferences data, have been criticized for their failure to replicate real life situations and because they may suffer from hypothetical bias as there is no guarantee that what a person indicates in the survey/interview, is what he/she would do in real life (Lusk et al., 2004).

Thus, using real market sales data, an objective metric to assess the market performance of a product's attribute, such as its implicit price (Rosen, 1974), should be used. Otherwise, an alternative strategy may be to use new data collection methods, such as virtual reality-based methods, leading to simulated purchase patterns highly correlated with actual sales data, and whose results are close to approximate real-life consumer behavior and mitigate hypothetical bias associated with stated preferences survey data (Burke, 1992; 1996; Campo, 1999). Furthermore, firms investing in developing and marketing functional products face additional hurdles, such as the compliance costs associated with the European Regulation (EC) No. $1924 / 2006$ as well as the uncertainty about the profitability of their investments in developing products with health claims.

Complying with the European Regulation (EC) No. 1924/2006 has been indicated as costly and associated with a high degree of uncertainty for firms, compared to other countries'

\footnotetext{
${ }^{3}$ Examples of such scales are: intention to try (Van Kleff et al., 2005a), healthiness perception (Ares and Gambaro, 2007; Ares et al., 2009), willingness to try (Ares and Gambaro, 2007; Ares et al., 2009), willingness to buy (Siegrist et al., 2008),perceived fit (Krutulyte et al., 2011), and attractiveness (Van Kleff et al., 2005a).
} 
regulatory frameworks, such as the USA and Canada's (Hobbs et al., 2014). Brookes (2010) collected data on the financial resources invested by food supplement manufacturers to comply with European Regulation (EC) No. 1924/2006. He estimated the average cost to develop and market a product with a "General function" claim ranging from 980 to 1,663 EUR; while, that of a product with a "Reduction of disease risk" claim ranges from 6,400 to 8,000 EUR plus the additional costs necessary to produce evidence from clinical trials to be included in the dossiers varying from 0.25 to more than 1 million EUR. These investments may be prohibitive for many food companies, as the EU food industry is characterized by low a level of financial resources and R\&D investments (Banterle et al., 2011).

Thus, if consumer willingness to pay for products with these attributes is low, firms may experience low returns from their investments in the development of functional products. Existing studies using survey data found mixed results about consumers' willingness to pay for health-enhancing features: while some authors found higher values of consumers' willingness to pay for these attributes (Ding et al., 2012; Balcombe et al., 2014; Moro et al., 2015), others found only a limited price premium (Menrad, 2003; Larue et al., 2004).

\subsection{Research objectives}

The general objective of this thesis is to assess quantitatively the implicit price of health claims, and to generate new knowledge that will inform food manufacturers on how to target consumers with high willingness to pay. The sub-objectives are the following:

1. To identify consumer-related factors affecting the acceptance of, and preference for, nutrition-modified and functional dairy foods products.

2. To estimate the implicit price of different types of health claims allowed by Reg. No.1924/2006, conditional on the main factors affecting consumer acceptance of these products, as suggested by the literature.

3. To investigate the existence of price dispersion due to asymmetric information between manufacturers and consumers that prevents firms to target consumers with a higher value of willingness to pay.

4. To assess the role of consumers' psychological traits, such as the individual level of body image satisfaction, on their decision to select functional products.

This thesis focuses on the functional dairy category, and in particular yogurts. Dairy represents one of the biggest market segment among nutrition-modified and functional products, accounting for nearly $43 \%$ of total worldwide sales (Ozer and Kirmaci, 2010). Furthermore, dairy products, and especially yogurts, are considered by consumers as among the most credible product carriers to host functional ingredients. Consumers' acceptance of, and preferences for nutrition-modified and functional dairy products have been widely investigated (inter alia, Van Kleff et al., 2005; Ares and Gambaro, 2007; Krutulyte et al., 
2008; Siegrist et al., 2008; Sirò, 2008; Ares et al., 2010). Also, the yogurt category is highly differentiated in term of health-enhancing features (Bonanno, 2012).

Research question 1 is answered reviewing systematically the literature on consumer acceptance of, and preferences for nutrition-modified and functional dairy products published in English language peer-reviewed journals from 1999 to 2013.

Research questions 2 and 3 are answered using scanner data of Italian yogurt sales. The data come from SymphonyIRI Group and are provided by the University of Foggia. The data contain information about monthly yogurt sales collected at the point-of-sale (hypermarkets and supermarkets checkouts), and then aggregated at the regional level, encompassing a 25month period between November 29, 2010, and December 31, 2012, for the entire Italian market. These scanner data are recorded at the European Article Number (EAN) level ${ }^{4}$. These data contain information on volume sold and value of sales, price (EUR/L), percentage of store selling the products, number of items sold in the store and several product characteristics, ranging from brand to functionality.

Research question 4 is answered with the use of consumer data collected through a Computer Aided Web Interviewing (C.A.W.I.) survey. The recruited participants were first asked to select from a virtual yogurt shelf the same yogurts they purchase habitually in the store. The software simulating the virtual shelf also recorded the number of functional yogurt packages that each participant purchased "virtually".

\subsection{Thesis outline}

The remainder of the thesis is composed of four research chapters, with each chapter aimed at achieving one of the four research objectives discussed above (Chapter 2-5), and a last chapter with a general discussion and main conclusions (Chapter 6).

Chapter 2 reviews systematically the literature on consumer acceptance of, and preferences for nutrition-modified and functional dairy foods. The review assesses the findings of existing studies and characterizes what consumer- and product-related characteristics affect consumers' acceptance of, and preferences for such classes of products, identifying also gaps in the literature.

Chapter 3 estimates the implicit price of health claims available in the Italian yogurt market allowed by Reg. No.1924/2006. The implicit price of each claim is decomposed according to three main factors: health claims' type, efficacy, and brand familiarity. The results presented in chapter 3, indicate that these factors affect the implicit prices of health claims in the Italian yogurt market.

\footnotetext{
${ }^{4}$ The EAN barcodes are 13-digit codes used in the coding system at the retail level and are used in Europe for identifying products whose barcodes are read by scanner cash registers at the point of sale where products are sold.
} 
Chapter 4 explores manufacturers' ability to successfully differentiate their products in a market where asymmetric information may potentially generate price dispersion, impairing firms' ability to price their products systematically at higher levels. The results indicate that firms that are able to signal higher quality by means of credence attributes can reach consumers with higher willingness to pay for the different attributes bundles they offer.

Chapter $\mathbf{5}$ assesses the role of an individual psychological trait, body image, on the selection of health-enhancing food options from a virtual supermarket shelf. A body image dissatisfaction was found inversely related with respondents' number of functional food packages purchased.

Chapter 6 synthetizes the main findings of the thesis, outlines the policy and business implications of the main results, highlights limitations, future research directions, and summarizes the main conclusions.

\section{References}

Annunziata, A. and Vecchio, R. (2013). Consumer perception of functional foods: A conjoint analysis with probiotics. Food Quality and Preference 28: 348-355.

Ares, G. and Gámbaro, A. (2007). Influence of gender, age and motives underlying food choice on perceived healthiness and willingness to try functional foods. Appetite 49: 148-158.

Ares, G., Besio, M., Giménez, A. and Deliza, R. (2010). Relationship between involvement and functional milk desserts intention to purchase. Influence on attitude towards packaging characteristics. Appetite 55: 298-304.

Ares, G., Giménez, A. and Gámbaro, A. (2009). Consumer perceived healthiness and willingness to try functional milk desserts. Influence of ingredient, ingredient name and health claim. Food Quality and Preference 20: 50-56.

Balcombe, K., Bitzios, M., Fraser, I. and Haddock-Fraser, J. (2014). Using attribute importance rankings within discrete choice experiments: an application to valuing bread attributes. Journal of Agricultural Economics 65(2): 446-462.

Banterle, A., Cavaliere, A., Carraresi, L. and Stranieri, S. (2011). Innovativeness in food small business: What is its relationship with marketing? Agricultural Economic Czech Republic 57: 474-483.

Bech-Larsen, T. and Scholderer, J. (2007). Functional foods in Europe: consumer research, market experiences and regulatory aspects. Trends in Food Science and Technology 118: 231-234.

Bigliardi, B. and Galati, F. (2013). Innovation trends in the food industry: The case of functional foods. Trends in Food Science \& Technology 31: 118-129. 
Bimbo F., Bonanno, A. and Viscecchia R. (2016). Do health claims add value? The role of functionality, effectiveness and brand. European Review of Agricultural Economics 43(5): 761-780.

Bleiel, J. (2010). Functional foods from the perspective of the consumer: How to make it a success? International dairy journal 20(4): 303-306.

Bonanno, A. (2012). Some like it healthy: demand for functional and conventional yogurts in the Italian market. Agribusiness: an International Journal 28(1): 67-85.

Brookes, G. (2010). Economic Impact Assessment of the European Union (EU)'s Nutrition\& Health Claims Regulation on the EU Food Supplement Sector and Market. European Health Claims Alliance EHCA.

Burke, R. R. (1996). Virtual shopping: Breakthrough in marketing research. The Journal of Product Innovation Management 6(13): 558-559.

Burke, R. R., Harlam, B. A., Kahn, B. E. and Lodish, L. M. (1992). Comparing dynamic consumer choice in real and computer-simulated environments. Journal of Consumer Research 71-82.

Campo, K., Gijsbrechts, E. and Guerra, F. (1999). Computer simulated shopping experiments for analyzing dynamic purchasing patterns: Validation and guidelines. Journal of Empirical Generalisations in Marketing Science 4(2): 22-61.

Caswell, J. A. and Mojduszka, E. M. (1996). Using informational labeling to influence the market for quality in food products. American Journal of Agricultural Economics 78(5): 1248-1253.

Caswell, J. A. and Padberg, D.I. (1992). Toward a more comprehensive theory of food labels. American Journal of Agricultural Economics 74(2): 460-468.

Cox, D.N., Evans, G. and Lease, H.J. (2007). Predictors of Australian consumers' intentions to consume conventional and novel sources of long-chain omega-3 fatty acids. Public Health Nutrition 11(1): 8-16.

Darby, MR. and Karni, E. (1973). Free competition and the optimal amount of fraud. Journal of Law Economics 16:67-88.

Dee, A., Kearns, K., O’Neill, C., Sharp, L., Staines, A., O’Dwyer, V., ... and Perry, I. J. (2014). The direct and indirect costs of both overweight and obesity: a systematic review. BMC research notes 7(1): 242.

Devcich, D. A., Pedersen, I. K. andPetrie, K. J. (2007). You eat what you are: Modern health worries and the acceptance of natural and synthetic additives in functional foods. Appetite, 48: 333-337.

Ding, Y., Veeman, M. M. and Adamowicz, W.L. (2012). The influence of attribute cutoffs on consumers' choices of a functional food. European Review of Agricultural Economics 39(5): 745-769. 
Diplock, A.T., Aggett, P.J., Ashwell, M., Bornet, F., Fern, E.B. and Roberfroid, M.B. (1999). Scientific concepts of functional foods in Europe: consensus document. British Journal of Nutrition 81: 1-27.

Grunert, K. G. (2005). Food quality and safety: consumer perception and demand. European Review of Agricultural Economics 33: 369-391.

Hobbs, J. E., Malla, S. and Sogah, E. K. (2014). Regulatory frameworks for functional food and supplements. Canadian Journal of Agricultural Economics/Revue Canadienne d'agroeconomie 62(4): 569-594.

Krutulyte, R., Grunert, K. G., Scholderer, J., Lähteenmäki, L., Hagemann, K. S., Elgaard, P., Nielsen, B. and Graverholt, J. P. (2011). Perceived fit of different combinations of carriers and functional ingredients and its effect on purchase intention. Food Quality and Preference 22(1): 11-16.

Krutulyte, R., Grunert, K.G., Scholderer, J., Hagemanna, K.S., Elgaarda, P., Nielsena, B. and Graverholt, J.P. (2008). Motivational factors for consuming omega-3 PUFAs: An exploratory study with Danish consumers Appetite 5(1): 137-147.

Labrecque, J., Doyon, M., Bellavance, F. and Kolodinsky, J. (2006). Acceptance of Functional Foods: A Comparison of French, American, and French Canadian Consumers. Canadian Journal of Agricultural Economics/Revue canadienned'agroeconomie 54: 647661.

Larue, B., West, G. E., Gendron, C. and Lambert, R. (2004). Consumer response to functional foods produced by conventional, organic, or genetic manipulation. Agribusiness 20(2): 155166.

Lusk, J.L., Feldkamp, T. and Schroeder, T. C. (2004). Experimental Auction Procedure: Impact on Valuation of Quality Differentiated Goods'. American Journal of Agricultural Economics 86: 389-405.

Menrad, K. (2003). Market and marketing of functional food in Europe. Journal of Food Engineering 56(2): 181-188.

Moro, D., Veneziani, M., Sckokai, P. and Castellari, E. (2015). Consumer willingness to pay for catechin enriched yogurt: evidence from a stated choice experiment. Agribusiness: An International Journal 31(2): 243-258.

Nutraingredients-US. US leads in healthy food product launches, 27 Aug 2009. http://www.nutraingredients-usa.com/Markets/US-leads-in-healthy-food-product-launches

Ozer, B.H. and Kirmaci, H.A. (2010). Functional milks and dairy beverages. International Journal of Dairy Technology 63: 1-15.

Regulation of European Commission (EU) No 1924/2006 of the European Parliament and of the Council of 20 December 2006 on nutrition and health claims made on foods. European Commission, EUR 23380 EN. Luxemburg: European Communities. 
Réquillart, V. and Soler, L. G. (2014). Is the reduction of chronic diseases related to food consumption in the hands of the food industry?. European Review of Agricultural Economics 41(3): 375-403.

Research and Markets (2014). Global Functional Food and Nutraceuticals Market (2014 2020) - By Type (Foods, Beverages, Supplements); Benefits (Health and Wellness, Disease Prevention, Fitness, Beauty); Origin \& Ingredient.

Roe, B. and Sheldon, I. (2007). Credence Good Labeling: The Efficiency and Distributional Implications of Several Policy Approaches. American Journal of Agricultural Economics 89 (4): 1020-1033.

Rosen, S. (1974). Hedonic prices and implicit markets: Product differentiation in pure competition. Journal of Political Economy 82: 34-55.

Siegrist, M., Stampfli, N. and Kastenholz, H. (2008). Consumers' willingness to buy functional foods. The influence of carrier, benefit and trust. Appetite 51: 526-529.

Siró, I., Kápolna, E., Kápolna, B. and Lugasi, A. (2008). Functional food. Product development, marketing and consumer acceptance—a review. Appetite 51: 456-467.

Stein, A.J., Rodrfguez-Cerezo E. (2008). European Commission- Joint Research Centre Institute for Prospective Technological Studies. Functional Food in the European Union.

Teisl, M. F. and Roe, B. (1998). The economics of labeling: an overview of issues for health and environmental disclosure. Agricultural and Resource Economics Review 27: 140-150.

Traill, B.W. (2012). Presidential Address: Economic Perspectives on Nutrition Policy Evaluation. Journal of Agricultural Economics 63(3): 505-527.

Urala, N. and Lähteenmäki, L. (2004). Attitudes behind consumers' willingness to use functional foods. Food Quality and Preference 15: 793-803.

Urala, N. and Lähteenmäki, L. (2007). Consumers' changing attitudes towards functional foods. Food Quality and Preference 18: 1-12.

Van Kleef, E., van Trijp, H., Luning, P. and Jongen, W.M. (2002). Consumer-oriented functional food development: how well do functional disciplines reflect the 'voice of the consumer'?. Trends in Food Science \& Technology 13(3): 93-101.

Van Kleef, E., van Trijp, H.C.M. and Luning, P. (2005a). Functional foods: health claim-food product compatibility and the impact of health claim framing on consumer evaluation. Appetite 44: 299-308.

Van Kleef, E., van Trijp, H.C.M. and Luning, P. (2005b). Consumer research in the early stages of new product development: a critical review of methods and techniques. Food quality and preference 16(3): 181-201.

Verbeke, W. (2005). Consumer acceptance of functional foods: socio-demographic, cognitive and attitudinal determinants. Food quality and preference 16(1): 45-57. 
Chapter 1

WHO (2010). Global Status Report on Non-communicable Diseases. Geneva: World Health Organization.

Wong, A.Y.T., Lai, J.M.C. and Chan, A.W.K.. (2015). Regulations and protection for functional food products in the United States. Journal of Functional Foods 17: 540-551. 


\section{Chapter 2}

\section{Consumer acceptance and preferences for functional dairy products: a}

systematic review.

Francesco Bimbo, Alessandro Bonanno, Giuseppe Nocella, Rosaria Viscecchia, Gianluca Nardone, Biagia De Devitiis, Carlucci Domenico.

Paper published on Appetite, Volume 113, 1 June 2017, Pages 141-154 https://doi.org/10.1016/j.appet.2017.02.031 


\title{
Consumers' acceptance and preferences for nutrition-modified and functional dairy products: a systematic review.
}

\begin{abstract}
This systematic literature review collects and summarizes research on consumer acceptance and preferences for nutrition-modified and functional dairy products, to reconcile, and expand upon, the findings of previous studies. We find that female consumers show high acceptance for some functional dairy products, such as yogurt enriched with calcium, fiber and probiotics. Acceptance for functional dairy products increases among consumers with higher diet/health related knowledge, as well as with aging. General interest in health, foodneophobia and perceived self-efficacy seem also to contribute shaping the acceptance for functional dairy products. Furthermore, products with "natural" matches between carriers and ingredients have the highest level of acceptance among consumers. Last, we find that brand familiarity drives consumers with low interest in health to increase their acceptance and preference for health-enhanced dairy products, such as probiotic yogurts, or those with a general function claim.
\end{abstract}

Keywords: nutrition-modified and functional dairy products, systematic review, consumers' acceptance and preferences, attitudes, perceived healthiness.

\section{Introduction}

In the last decades consumer demand for health-enhancing food products, such as nutritionmodified (e.g. low-fat products or with fiber added) and functional foods, has grown rapidly. Consumer demand for health-enhancing foods has spurred in part because of socio-economic changes, such as the longer life expectancy, the rise of health care costs, the social costs of non-transmittable diseases, and the widespread desire for a better quality of life (Valls et al., 2013).

A recent report estimates that the global market for foods with health-enhancing features amounted to (approximately) $\$ 168$ billion in 2013, with an annual average growth rate of $8.5 \%$, and it is forecasted to exceed $\$ 300$ billion by 2020 (Research and Markets, 2014). Food companies, attracted by such market growth and high margins, have been investing in the development of new nutrition-modified and functional products (Khan et al., 2014).

However, these market projections mask a high risk of product failure as 70 to 90 per cent of new health-enhancing products exit the market within the first two years from their launch (Heasman \& Mellentin, 2001; Stein \& Rodríguez-Cerezo, 2008; Hardy, 2010). One of the likely reasons for such high failure rates is that product development is often driven by technical feasibility (Bleiel, 2010) disregarding consumers' acceptance and preferences (Van Kleef et al., 2002; 2005a). This approach may lead to a mismatch between consumers' needs 
and the features of new nutrition-modified and functional food products introduced in the market (Van Kleef et al., 2002). In spite of existing research having given great emphasis to consumers acceptance and preferences towards nutrition-modified and functional foods (Van Kleef et al., 2002; Verbeke, 2005; Ares \& Gámbaro, 2007), existing knowledge is fragmented, and the findings from studies conducted in different contexts appear difficult to reconcile. One likely reason for this difficulty may be that so far scholars have focused on only one or just a few aspects of consumer behavior, thus failing to provide an integrated picture of the multiple elements affecting the acceptance and the preferences for these products (Starling, 2014).

One approach used to gather relevant knowledge in fields where evidence is fragmented is the systematic review, which selects studies through a multi-step procedure (Cooper, 1998; Littell \& College, 2006), also allowing for an assessment of the studies' quality (Littell et al., 2008). To the best of our knowledge, only two systematic reviews on functional foods exists (Ozen et al., 2012; Ozen et al., 2014). Ozen et al. (2012) systematically reviewed twenty-three worldwide studies on individual consumption of functional products belonging to different food categories. These authors concluded that it was not possible to clearly identify how gender, age, level of education and socio-economic characteristics influenced the consumption of functional foods. Similarly, Ozen et al. (2014), by systematically reviewing studies on European consumers, failed to identify gender differences in the individual consumption of many categories of nutrition-modified and functional foods; however, these authors pointed out a higher consumption of such products among North European consumers.

The contradictory findings highlighted in these systematic reviews may be due to the authors considered studies that focused on different products, for which consumers' acceptance and preferences may be inherently different. Thus, the different attitudes shown by consumers across product categories may have played the role of a confounding factor, impairing the authors' possibility to isolate patterns characterizing consumption. Such heterogeneity in results conflicts also with other literature reviews (such as Sirò et al., 2008; Lähteenmäki, 2013) which have instead found specific patterns in the role of consumer-related characteristics, such as gender, age, and some psychological variables, as well as a clear role of product-related characteristics in shaping consumers acceptance for nutrition-modified and functional products. The primary goal of this paper is to investigate if, by focusing in one specific product category, dairy products, it is possible to isolate common patterns in consumers' acceptance and preferences for nutrition-modified and functional foods by means of a systematic review process. Our secondary goal is also to provide an integrated picture of the multiple elements affecting the acceptance and preferences for dairy products. We chose dairy products as the category of interest for two reasons. First, dairy products are one of the biggest market segment among nutrition-modified and functional products, accounting for nearly $43 \%$ of the total worldwide sales (Ozer \& Kirmaci, 2010). Second, dairy products are considered by consumers as one of the most credible product carriers to host functional ingredients, and consumers' acceptance and preferences towards nutrition-modified and 
functional dairy have been largely investigated in literature (inter alia, Van Kleff et al., 2005; Ares \& Gambaro, 2007; Krutulyte et al., 2008; Siegrist et al., 2008; Sirò, 2008; Ares et al., 2010).

Gaining more insight on consumers' preferences for a wide range of heath-enhancing dairy products may benefit both dairy manufacturers and consumers, as it will be illustrated throughout the manuscript. Furthermore, the results of this review, along with its limitations, will help identifying avenues for future research, as it will be illustrated in the final section of this article.

\section{Methods}

We used a systematic literature review methodology for the social sciences to select articles from online academic search engines. Compared to narrative reviews, the systematic literature review technique has the advantage of being based on an explicit and accurate study selection process which involves a multi-step procedure similar to that used in research surveys (Cooper, 1998; Littell \& College, 2006). Additionally, the systematic review process required findings to be weighted according to the quality of the study they originate from; therefore an ad hoc quality assessment protocol was built, based upon recommendations on how to assess social science papers (Littell et al., 2008).

Studies selection

An initial inventory of relevant online databases was created. Scopus, ScienceDirect, and Google Scholar were identified as search engines from which to retrieve the studies to be included in the review. Google Scholar, ScienceDirect and Scopus were selected as they use different approaches to index documents available on the internet. Since ScienceDirect and Scopus only index title, abstract and keywords documents containing search terms and keywords in the main text cannot be retrieved during the search process from those web engines. Instead, Google Scholar can select larger amount of documents compared to the other two search engines, as it indexes the documents' main text. Thus, by using them jointly the likelihood of retrieving articles related to the subject being investigated can be maximized (Ford, 2011).

The search process was restricted to research papers published in English in peer reviewed journals from 1999 to 2013. The choice of this time span was motivated by the fact that nutrition-modified and functional products started to be introduced in the market approximately at the end of the last century (Sirò et al., 2008) and by the time when the articles were collected (November 2013).

As illustrated in figure 1, the selection process continued with three steps in which inclusion/exclusion criteria reduced the number of studies gradually, by means of structured queries developed using Boolean operators and two sets of keywords. 
Chapter 2

Figure 1. Selection papers process.

Queries used:

1) “cheese" OR "yogurt" OR "butter" OR "milk" OR "spreadable" OR "functional food" AND "consumer";

2) "low fat" OR "light" OR "low salt" OR "vitamin" OR "omega-3" OR "fatty acid" OR "CLA" OR "calcium" OR "antioxidant" OR "probiotic" OR "prebiotic" OR "fibre" OR "functional food" AND "consumer".
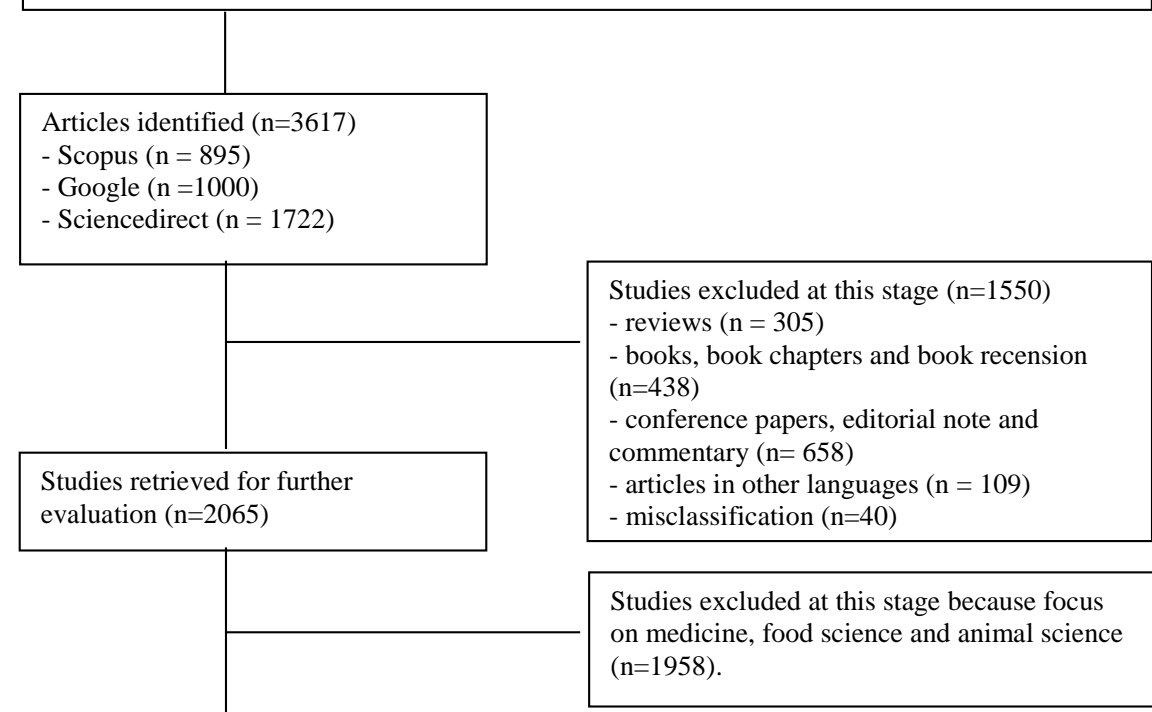

Studies retrieved for titles, abstract and full text analysis $(n=109)$

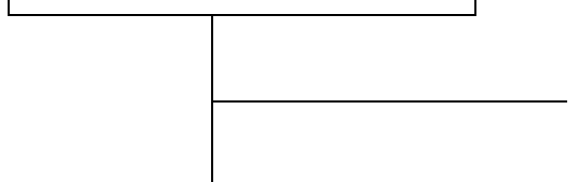

Studies excluded at this stage $(n=67)$

- duplicated studies $(n=31)$

- sensorial profile of dairy products without

information about consumer behavior $(n=36)$

Studies included in this systematic review $(n=42)$ 
The first set of keywords included terms referring to the most frequently consumed nutritionmodified and functional dairy products according to Sirò (2008): "cheese", "yogurt", "butter", "milk" and "spread". The second set of keywords included the terms: "functional food", "vitamin", “omega-3", "fatty acid", "CLA" (Conjugated Linoleic Acid), “calcium", "antioxidant", "probiotic", "prebiotic", "fiber", "low fat", "light" and "low salt", which refer to the health-related attributes most frequently attached to dairy products (Playne et al., 2003; Sirò, 2008). Finally, the term "consumer" was added to the queries to identify only studies focusing on heath-enhancing dairy products and consumers.

The search output initially included 3,617 articles: 895 identified via Scopus, 1,000 via Google Scholar, and 1,722 via ScienceDirect. In the first step, the language of the study and the type of publications (e.g. research papers, reviews, and books) were used as selection criteria. In the second step, titles and abstracts of the remaining 2065 papers were inspected, retaining only those focusing on issues related to consumer behavior and nutritionmodified/functional foods. In the third step, the remaining 109 studies were further reduced by excluding 31 studies that were duplicates, and 36 which focused on the sensory profiles of these products without assessing aspects related to consumer behavior. It is worth pointing out that more than half of the 42 articles identified to be reviewed appeared multiple times among the final set of 109 papers: as the same paper was retrieved by two or all of the three search engines at the beginning of search process. The final list of the 42 articles identified to be included in this review is reported in table 1.

\section{Quality Assessment}

The quality assessment procedure is one of the steps in the systematic literature review process differentiating it from other types of reviews (Littell \& College 2006; Littell et al., 2008). This step requires the use of specific criteria to create a quality score for each of the studies identified, and to produce a ranking of their quality. The quality assessment was not easy to perform given the high heterogeneity of the methodological approaches employed in this research domain, and because of the lack of standardized quality assessment tools for studies belonging to the social science field.

Therefore, similarly to Cox et al. (2015), an ad hoc quality assessment tool was developed using the Instrument Critical Appraisal Checklist (2009) provided by the Joanna Briggs Institute as a reference document.

This quality assessment protocol consists of six criteria, identified according to the authors' expertise (Appendix table A.1.).

The first criterion considered whether the analysis performed was qualitative or quantitative in nature. The adequacy of the sample size used and whether the sample was representative of a specific population group were the second and third criteria considered. The remaining three criteria were whether the study included a theoretical framework, whether confounding factors and biases were accounted for in the empirical analysis performed, and if the outcome variable of the study was measured using a validated measure and/or one objectively 
Chapter 2

Table 1. Summary of the studies, quality ranking and research area covered.

\begin{tabular}{|c|c|c|c|c|c|c|}
\hline \multirow[b]{2}{*}{ Study } & \multirow[b]{2}{*}{ Quality } & \multicolumn{5}{|c|}{ Area covered } \\
\hline & & Gender & Age & $\begin{array}{l}\text { Diet-health } \\
\text { knowledge and } \\
\text { lifestyle }\end{array}$ & $\begin{array}{l}\text { Perceived healthiness } \\
\text { and product attributes }\end{array}$ & $\begin{array}{l}\text { Psychological } \\
\text { factors }\end{array}$ \\
\hline Almli et al. (2011) & Medium & & & & & $\mathrm{X}$ \\
\hline $\begin{array}{l}\text { Annunziata \& Vecchio } \\
\text { (2013) }\end{array}$ & High & $\mathrm{X}$ & & $\mathrm{X}$ & $\mathrm{x}$ & \\
\hline Ares \& Deliza (2010) & Low & & & & $\mathrm{X}$ & \\
\hline Ares \& Gambaro (2007) & Medium & $\mathrm{X}$ & & & $\mathrm{X}$ & \\
\hline Ares et al.(2008) & Low & & & $\mathrm{X}$ & $\mathrm{X}$ & \\
\hline Ares et al.(2009) & Medium & $\mathrm{X}$ & $\mathrm{X}$ & & $\mathrm{X}$ & \\
\hline Ares et al.(2010a) & Medium & $\mathrm{X}$ & & & $\mathrm{X}$ & \\
\hline Ares et al.(2010b) & Medium & $\mathrm{X}$ & & & $\mathrm{X}$ & \\
\hline $\begin{array}{l}\text { Barrena \& Sanchez } \\
\text { (2010) }\end{array}$ & Low & & & $\mathrm{x}$ & $\mathrm{x}$ & $\mathrm{x}$ \\
\hline Barrios et al. (2007) & Low & & & $\mathrm{X}$ & & \\
\hline $\begin{array}{l}\text { Bech-Larsen \& Grunert } \\
(2002)\end{array}$ & High & & & & $\mathrm{x}$ & \\
\hline Bonanno (2012) & High & & $\mathrm{X}$ & & & \\
\hline Chase et al.(2009) & High & & $\mathrm{X}$ & $\mathrm{X}$ & $\mathrm{X}$ & \\
\hline Cox et al. (2007) & High & & & & & $\mathrm{x}$ \\
\hline Cox et al.(2011) & High & $\mathrm{X}$ & & & & \\
\hline de Jong et al. (2003) & High & & $\mathrm{X}$ & $\mathrm{X}$ & & \\
\hline Grunter et al. (2000) & Medium & & & & & \\
\hline Hailu et al. (2009) & Medium & $\mathrm{X}$ & $\mathrm{X}$ & $\mathrm{X}$ & $\mathrm{X}$ & \\
\hline Johansen et al. (2011) & Medium & $\mathrm{X}$ & & & $\mathrm{X}$ & \\
\hline $\begin{array}{l}\text { Kahkonen \& Tuorila } \\
\text { (1999) }\end{array}$ & Low & & & & $\mathrm{x}$ & \\
\hline Krutulyte et al. (2008) & High & & & & $\mathrm{X}$ & \\
\hline Krutulyte et al. (2011) & High & & & & $\mathrm{x}$ & \\
\hline Labrecque et al. (2006) & High & & & $\mathrm{x}$ & & $\mathrm{x}$ \\
\hline $\begin{array}{l}\text { Lähteenmäki et } \\
\text { al.(2010) }\end{array}$ & High & & & & $\mathrm{X}$ & \\
\hline Landström et al. (2007) & High & $\mathrm{X}$ & $\mathrm{X}$ & $\mathrm{X}$ & & $\mathrm{x}$ \\
\hline Landström et al. (2009) & Low & & & $\mathrm{X}$ & & \\
\hline Marette et al. (2010) & High & & & & $\mathrm{x}$ & \\
\hline Maynard (2005) & Low & & & & $\mathrm{x}$ & \\
\hline Messina et al.(2008) & High & & $\mathrm{X}$ & & $\mathrm{X}$ & \\
\hline Mireaux et al. (2007) & Low & & & & & \\
\hline Mullie et al. (2013) & High & & $\mathrm{X}$ & & & \\
\hline O'Brien et al. (2012) & High & & & & $\mathrm{x}$ & \\
\hline$\emptyset$ vrum et al. (2012) & High & & $\mathrm{X}$ & $\mathrm{X}$ & $\mathrm{X}$ & \\
\hline Peng et al. (2006) & Medium & $\mathrm{X}$ & $\mathrm{X}$ & & $\mathrm{x}$ & \\
\hline Siegrist et al. (2008) & High & $\mathrm{X}$ & $\mathrm{X}$ & & & $\mathrm{x}$ \\
\hline $\begin{array}{l}\text { Urala \& Lähteenmäki } \\
\text { (2004) }\end{array}$ & High & & $\mathrm{X}$ & & & $\mathrm{x}$ \\
\hline $\begin{array}{l}\text { Urala \& Lähteenmäki } \\
\text { (2007) }\end{array}$ & High & $\mathrm{X}$ & $\mathrm{X}$ & & & $\mathrm{x}$ \\
\hline van Kleef et al. (2005a) & High & & & $\mathrm{X}$ & $\mathrm{X}$ & \\
\hline Viana et al., (2008) & Low & & & $\mathrm{x}$ & & \\
\hline Wahba et al. (2006) & Medium & & & $\mathrm{X}$ & & \\
\hline Williams et al. (2008) & Low & & & & $\mathrm{X}$ & \\
\hline Zandstra et al. (2001) & Medium & & & $\mathrm{X}$ & & $\mathrm{X}$ \\
\hline
\end{tabular}

quantifiable (e.g. probability to observe an outcome, willingness to pay, Likert scale). For more details see table A.1 in the Appendix.

The studies identified were rated as low, medium, or high quality, based upon a combination of the scores assigned to each of the six assessment criteria; equal weighting was given to 
each criterion. A study was considered as "high quality" if it rated "high" on three or more criteria; "medium quality," if it received two "high" or one "high" and two "medium"; the remaining studies were classified as "low quality." For a complete list of the papers' scores in all the criteria and their overall quality rating, see table A.2 in the Appendix.

\section{Results}

Table 1 presents a summary of the identified studies' features. The majority of the studies identified ( 23 out of 42) were ranked as "high" quality, whereas, about three quarters (32 out of 42), were ranked as either "high" or "medium" quality. The majority of the studies, circa $80 \%$, were published between 2004 and 2013. Northern Europe, North America and Uruguay were the geographical areas most investigated. In terms of research design, 26 are single cross-sectional studies and show an average sample size of 504 observations, with a minimum number of observations of 50 and a maximum of 2,269;8 studies are multiple cross-sectional studies, with sample sizes ranging from 96 to 5,967 observations, for an average of 1,602; two are longitudinal studies, one is a cohort study, and the remaining studies are based on exploratory research design (focus group interviews). The age of the consumers interviewed ranges from 14 to 90 years of age, with one study only focusing on consumers below the age of 30 , and another on consumers above 65 years of age.

Generally speaking, the studies identified investigate aspects of consumer behavior by comparing two or more food carriers delivering different health-related properties. The most frequently investigated dairy food carrier, that is, the vehicle where bioactive ingredients can be incorporated or modified (e.g. beverages, bread, cereal, margarine, eggs), was yogurt (30 articles), followed by milk (11), cheese (10) and milk desserts (4). With regard to the healthrelated attributes, probiotic, 'low fat content', and omega-3 were the most studied (11 articles), followed by antioxidants (5), fiber (4), calcium (4), vitamins (2) and iron (1).

In terms of the data analysis techniques used, most of the studies adopted multivariate analysis techniques, such as analysis of variance or regression analysis. Data reduction techniques, like cluster analysis and principal component analysis, were employed in 9 out of 42 studies as intermediate techniques to identify consumers' market segments on which to perform further analysis. For more details on the features of the studies included in this review, see table A.2 in the Appendix.

\subsection{Consumer related characteristics}

\section{Gender}

The studies reviewed show the existence of a gender dimension in the acceptance and preference for nutrition-modified and functional dairy products, with most studies highlighting that women have higher levels of acceptance than men. Most of the findings related to gender come from medium and high quality studies. For example, Johansen et al. (2011) found more positive attitudes for low fat dairy products such as yogurt and cheese among Norwegian, Danish and Californian female consumers, compared to men. High 
female acceptance was mainly due to the fact that low-fat products supported weight-control needs of many women which are, on average, also more health consciousness than men (Wardle et al., 2014). Ares \& Gambaro (2007) and Ares et al. (2009) pointed out that female consumers attached the highest values of willingness to try yogurts with added fiber or calcium. These dairy products were highly accepted compared to other functional concepts. Furthermore, female consumers showed positive attitudes for a functional dessert using milk as a base product (Ares et al., 2009), and a higher acceptance was especially recorded among individuals with a high level of personal involvement with the product (Ares et al., 2010a). A similar result was obtained by Hailu et al. (2009), who investigated a sample of Canadian consumers: these authors found that female consumers strongly prefer yogurt as a carrier to deliver probiotics rather than using pills or ice cream as a vehicle. Females' preferences for functional dairy products, especially for probiotic yogurt, also emerge from one high quality study performed by Annunziata and Vecchio (2013) on a representative sample of Italian consumers.

Other findings from high quality studies using self-reported and actual consumption data confirmed the presence of a gender dimension. Landström et al. (2007) pointed out that female Swedish consumers part of a focus group study, declared that they consume/purchase more functional products than males, with a significantly larger share of probiotic milk products. De Jong (2003) instead, using a multivariate type of analysis and a large dataset of actual consumption data from the Dutch population, found weak evidence that being female is positively associated with the consumption of yogurt with added lactic acid bacteria, while the same was not found for males.

However, few medium (Peng et al., 2006; Ares et al., 2010b) and high quality (Siegrist et al., 2008; Cox et al., 2011) studies, found no gender difference in the acceptance of yogurts added with Conjugated Linoleic Acid (CLA) or omega-3 (Peng et al., 2006; Cox et al., 2011), antioxidants (Ares et al., 2010b) and other unknown ingredients conferring risk reduction or general function features to yogurt (Siegrist et al., 2008). These results may be due to, respectively, a general lack of consumers' interest (regardless of gender), for yogurts added with CLA or omega-3 (as discussed in the next section); the lack of consumers' familiarity with the term "antioxidants"; and the suspicion for health claims not related to specific functional compounds. Generally speaking, product familiarity, trust, and suspiciousness, are elements strongly linked to the novelty aspect of health-enhancing food products and may affect their acceptance (Bower et al., 2003; Urala \& Lähteenmäki, 2007, Barrena \& Sanchez, 2010). Also, Urala \& Lähteenmäki (2007) found no gender difference in the acceptance of probiotic/stomach friendly yogurt and blood pressure lowering milk drinks, among Finnish consumers, a result which may not be valid outside the Finnish sample/population surveyed.

Thus, in the light of what is discussed above, the majority of the studies reviewed converge in indicating females as the most likely consumers of nutrition-modified and functional dairy products and particularly for products providing benefits linked to intestinal well-being, weight loss and bone health. Functional dairy products promoting bone health appear to be 
strongly preferred among females because of their higher risk (compared to males) of developing osteoporosis (Ares \& Gambaro, 2007; Hailu et al., 2009).

Age

There is a general consensus among scholars that being older is positively associated with a higher interest in dairy products with health-enhancing features, especially for functional products with disease risk reduction properties. High quality studies conducted by Urala \& Lähteenmäki $(2004 ; 2007)$ on a sample of Finnish consumers found that older respondents were more willing to use functional foods with claims to reduce the risk of a disease, such as blood pressure lowering milk drinks (Urala \& Lähteenmäki, 2004;2007). Older respondents seem to perceive these products as more rewarding than younger consumers, since they can help counteract health issues related to aging (Urala \& Lähteenmäki, 2007). The perceived reward from consuming functional foods, including functional dairy products, was indicated as highly predictive of the willingness to use them (Urala \& Lähteenmäki, 2004; 2007). This result was also confirmed by another high quality study by Messina et al. (2008) investigating a large cross-country sample of older consumers. These authors suggested that their results may be due to older consumers having been exposed longer to functional products compared to younger ones (Messina et al., 2008; Urala \& Lähteenmäki, 2004; 2007). Thus, older consumers have more knowledge and familiarity with functional dairy products and their effects on health, and are more likely to accept them.

Additional evidence, from high (Siegrist et al., 2008; Øvrum et al., 2012) and medium quality studies (Peng et al, 2006; Ares \& Gambaro, 2007; Ares et al., 2009), corroborates the existence of a relationship between aging consumers and higher acceptance of nutritionmodified and functional dairy products, confirmed by medium and high quality studies using both self-declared and actual purchase data. Mullie et al. (2012) recorded higher self-declared consumption of low fat dairy among older Belgian consumers. Also, de Jong et al. (2003) found that being 65 or older is associated with higher consumption of many functional products, including functional yogurt with lactic acid bacteria (de Jong et al., 2003). Both Bonanno's (2012) study using Italian actual purchase data, and Chase et al.'s (2009) study of Canadian consumers, found that consumers increase their demand for functional yogurts and omega-3 added dairy products as they grow older.

Younger consumers instead show overall higher acceptance for products enhancing some physiological functions, such as those improving general well-being or those that help prevent fatigue, compared to older respondents, as supported by a high (Urala \& Lähteenmäki, 2004) and a medium quality (Hailu et al., 2009) study. Only one study found no difference in the acceptance for functional dairy products among individuals belonging to different age groups (Landström et al., 2007).

In summary, the majority of studies identified in this systematic literature review suggests that older consumers are more likely to accept willing to try, and to include both nutritionmodified and functional dairy products in their diet. Older individuals may constitute, along 
with women, the group of consumers most receptive to such products, especially for functional products claiming to reduce the risk of diseases.

\section{Diet-health knowledge and lifestyles}

The studies reviewed found that variables related to consumer's level of knowledge about the relationships between health and nutrition (Ares et al., 2008, Øvrum et al., 2012) and in general to the consumer's nutritional knowledge, (Labrecque et al., 2006; Whaba et al., 2006; Viana et al., 2008; Barenna \& Sanchez, 2010) are good predictors of consumer acceptance of some dairy products, such as probiotic yogurts, low-fat products as well as products with added calcium, antioxidant and fiber. However, some of the studies reviewed did not use validated measures to assess consumers' knowledge, thus their results may need further validation by means of validated scales. For example, Ares et al. (2008) exploring the role of nutritional knowledge on the functional dairy acceptance, used an ad hoc modification of the Nutrition Knowledge Questionnaire, developed by Parmenter and Wardle (1999) without assessing its validity.

An additional hurdle in assessing the effect of consumers' diet-health related knowledge on the acceptance of (and preference for) functional dairy products is that many other factors can affect this relationship, for example family size. In families with young children (below 12 years of age) parents feel more responsible for their health (Barrios et al., 2008; Annunziata $\&$ Vecchio, 2013) and that may push them to acquire more nutritional-, diet- and healthrelated knowledge. A similar increase can arise in individuals who have had direct or indirect experience with illnesses, due to the enhanced receptiveness to information regarding diet and health related issues (Van Kleef, 2005a; Annunziata \& Vecchio, 2013). Given the many factors affecting diet and health-related knowledge, more analyses using multivariate analysis methods, including mediation analysis, may be needed to isolate the role of nutritional/diethealth knowledge on consumer acceptance of nutrition-modified and functional dairy products.

Lastly, evidence from high quality studies points to a general consensus for lifestyle variables (such as practicing sport and taking supplements) influencing the acceptance of nutritionmodified and functional dairy products, as "wellness oriented" consumers appear more willing to trade the taste of food for health benefits (Zandstra et al., 2001; Landström et al., 2007). Although, at first glance, the group of health oriented consumers may be seen as the ideal target for health-enhancing products, they represent only a niche market. Food manufacturers' efforts could otherwise be directed to improve the taste of functional and nutrition-modified dairy products as a means to enlarge their potential market and to reduce their price, which are often indicated as barriers to health-enhancing products' consumption (Frewer et al., 2003; Landstrom et al., 2009).

\section{Psychological factors}

Many of the studies reviewed explored how psychological factors, recorded through specific scales, can influence consumers' preferences for health-enhancing products. Among the studies surveyed, some investigated the role of consumers' attitudes towards health and taste, 
on the acceptance of nutrition-modified and functional dairy products, employing the health and taste scale originally developed by Roinenen et al. (1999).

Two high quality studies, conducted by Landström et al. (2007) and Zandstra, de Graaf, \& Van Staveren (2001) on samples of Swedish and Dutch consumers, respectively, found that consumers who scored higher values of the 'general health interest' and 'light product interest' scales, recorded higher consumption of low-fat dairy products, conversely to those scoring higher for 'craving for sweet'. Also, according to another high quality study conducted by Labrecque et al. (2006), the attitudes towards health and taste may also contribute to explain cross-cultural preferences toward milk with omega-3 between Canadian, French and American students, despite their low frequency of consumption.

Two successive high quality studies by Urala and Lähteenmäki (2004; 2007) argued that functional foods differ from "conventional" healthy foods and thus the general health scale was expected to be a weak predictor of consumers' functional food choices. Therefore, they developed and used seven scales to predict the willingness to consume selected functional foods. These authors found that the "perceived reward of improving your own health and performance" best predicted consumers' willingness to use milk added with calcium, blood pressure lowering milk drinks, and low-fat cheese. However, although the perceived reward from consuming functional foods may predict Finnish consumers' willingness to use functional dairy products, this result may not apply to other cultures, as culture and food habits vary across countries. Therefore, more cross-cultural studies are needed to confirm that perceived reward plays a role in predicting consumers' use of functional dairy products.

Furthermore, as some functional foods are created by adding a bioactive ingredient to a food carrier, adding an external ingredient can influence acceptance of the overall product. Scholars have investigated consumers' acceptance of new functional ingredients-dairy products combinations by using the food-neophobia scale, originally proposed by Pliner \& Hobden (1992). Empirical evidence from high quality studies shows that food-neophobia is negatively correlated with the consumers' willingness to buy probiotic yogurt, whereas it does not affect consumers' willingness to buy other non-dairy functional products (Siegrist et al., 2008). Also, Urala \& Lähteenmäki (2007) report that consumers' neophobia was negatively correlated with the willingness to use probiotic yogurts, but that it does not affect the use of other functional products, like cholesterol-lowering spreads or milk with claims to lower blood pressure. On the one hand, it is likely that food-neophobia may play a different role in relation to different combinations of functional ingredients and carriers. On the other hand, results may be confounded by the fact that, for consumers with high cholesterol blood level, there is a "virtual prescription" for cholesterol lowering products, and that medical applications have been found to suppress neophobia, or risk perception (Alevizos, Mihas \& Mariolis 2007). Therefore, Urala \& Lähteenmäki (2007) findings may be biased as they did not account for the existence of cholesterol related problems in any of their respondents.

Since products with health-enhancing features are of recent market introduction, the relationship between consumers' attitudes towards food innovation and the acceptance of 
such new products has been the object of investigation in some of the studies included in this review. Almli et al. (2011) conducted a cross-cultural study where French and Norwegian consumers were asked to state their preferences toward traditional cheese added with omega3. In neither country the addition of omega- 3 in traditional cheeses showed a positive effect on the willingness to buy such product. Even though the results from Almli et al. (2011) suggest the existence of consumers' aversion towards innovative health-food solutions, their results may be in part due to consumers' aversion to the match of omega-3 with dairy products, amply documented in the next section.

A different approach was employed by Cox, Evans \& Lease (2007), in their high quality study. Using a Protection Motivation Theory framework (Rogers et al., 1975), these authors found that perceived self-efficacy was the best predictor of the likelihood of purchasing milk with omega-3 among a sample of Australian consumers. Compared to other carriers containing omega-3, the authors found that omega-3-enriched milks were the least likely to be purchased (Cox, Evans \& Lease 2007). Also, a low quality study by Barrena and Sanchez (2010) used a means-end chain approach on a sample of sixty Spanish households to link their knowledge of bifidus added to yogurt and milk, to consequences and personal values related to this product, finding a major personal dimension in the purchase and consumption of bifidus-added dairy among households with children.

In summary, these studies find that psychological factors contribute to shape consumers' acceptance for nutrition-modified and functional dairy products. Consumers can become more interested in these products once they can perceive/believe in their health enhancing properties (for themselves and/or for people close to them). However, all the studies reviewed focus on North European consumers; therefore, research conducted in other Southern countries may be useful for food manufacturers as functional food markets are fast growing. For example, Italy saw the highest number of new healthy products launch among European Countries between 2005 and 2009 (Nutraingredients, 2009).

\subsection{Product related characteristics}

Models assessing consumer acceptance and preferences by accounting for product characteristics populate the literature, along with those that explored consumers' perceived healthiness of many combinations of carriers and ingredients.

\section{Intrinsic product characteristics}

Intrinsic product characteristics can be defined as any informational stimuli of the physical product which cannot be changed without altering the essence of the product itself (Poulson et al., 1996). In the case of nutrition-modified and functional foods, intrinsic product characteristics are given by the combination of the health-enhancing ingredient with the type of carrier used.

Scholars' interest in consumers' perceived healthiness toward nutrition-modified and functional foods was due to the fact that the latter is highly correlated with the market success of the product and it was found being influenced by both intrinsic and extrinsic product characteristics (discussed in the next session). Consumers' perceived healthiness is usually 
measured on a sevem-point Likert scale ranging from 1, 'not healthy', to 7, 'extremely healthy' (Bech-Larsen \& Grunert, 2003). The combinations of carriers and ingredients receiving the highest perceived healthiness scores are more likely to be accepted by consumers, and to succeed in the marketplace (Grunert, 2000; Bech-Larsen \& Grunert, 2003; Krutulyte et al., 2008, 2011; Johansen et al. 2011; Cox et al., 2011).

Several of the studies identified in this review have investigated the perceived healthiness of carriers, ingredients and their combinations. Studies with different quality levels show that the perceived healthiness of a dairy product largely depends upon the consumer's perceived healthiness of the carrier (Ares et al., 2008; Hailu et al., 2009); others (van Kleef et al., 2005a; Hailu et al., 2009; Johansen et al., 2011) pointed to yogurt being perceived as the healthiest carrier among those tested, perhaps because yogurt is perceived as intrinsically healthy.

Furthermore, a number of mostly high quality studies among those reviewed, also indicate that consumers show strong acceptance for selected ingredients such as calcium and fiber, and a more positive perceived healthiness of health-enhancing foods where the bioactive ingredient is "naturally added" or it is inherent to the carrier (Cox et al., 2011; Krutulyte et al., 2008, 2011). For example, yogurt with added calcium is perceived as healthier than yogurt with added fibers, antioxidants and iron (Ares \& Gambaro, 2007). Instead, yogurts added with omega- 3 are perceived negatively, since they are characterized by a combination perceived as less natural than, for example, omega-3 and fish products (Krutulyte et al., 2011). Additionally, consumers struggle to associate the fish taste of omega-3 with the sweetness of yogurt, and are skeptical of the potential off-flavors produced by the addition of such ingredient to yogurt (Krutulyte et al., 2011). Low consumer acceptance for dairy products added with omega-3 was also confirmed by Chase et al. (2009) using Canadian purchase data matched with household related information. They found that more than $90 \%$ of the 7,947 households surveyed never purchased omega-3 added products. However, moderate acceptance of omega- 3 modified dairy products was recorded among individuals who perceived the risk of conditions associated with a metabolic syndrome (O'Brien et al., 2012).

Limited evidence exists, from medium and high quality studies, in support of the effectiveness of adding "external" ingredients to products considered unhealthy in order to improve their acceptance. In some cases, carriers with an unhealthy image, such as cheeses or spreads, known for their high cholesterol content, were perceived as good carriers for bioactive ingredients such as polyunsaturated fat or omega-3, mitigating the negative effect of cholesterol on health (Bech-Larsen \& Grunert, 2003; Peng et al., 2006). In these cases consumers may simply prefer health-enhancing dairy products whose bioactive ingredients "enhance" the innate or intrinsic properties of the product without altering its sensory characteristics, regardless on whether the ingredient is a "natural" addition to the carrier or it is exogenous to it. 
Given the findings presented above, there appears to be a widespread consensus in the literature that a "natural" match between added ingredient and carrier increases the overall acceptance of functional dairy products with health-enhancing features.

\section{Extrinsic product characteristics}

Extrinsic product characteristics are informational stimuli which are not physically part of the product, e.g. a product's label and its elements (Grunert et al., 1996). In the case of food products with health-enhancing features, extrinsic attributes are nutrition and health claims available on the labels, a product's brand, and its package. These characteristics work usually as tools to inform consumers about the product's properties, and to attract and influence shoppers' purchasing decisions. The existing literature provides conflicting results on how nutrition and health claims affect consumers' acceptance of nutrition-modified and functional dairy products (Bech-Larsen \& Grunert, 2003; Ares et al., 2009; Ares et al., 2010b). A medium and a high quality study identified in this systematic review suggest that individuals prefer dairy food products with health and nutrition claims rather than identical ones without a claim, suggesting that the presence of a claim increases the healthiness perception of products and therefore their acceptance (Bech-Larsen \& Grunert, 2003; Ares et al., 2009). A high quality study by Lähteenmäki et al. (2010) found no effect, or a slightly negative one, of the presence of health claims on consumer perceived healthiness by investigating a large sample of north European consumers.

Results of high quality studies indicate that the presence of nutrition and health claims may guide some groups of consumers in making healthier food choices (Marette et al., 2010; Øvrum et al., 2012), and that these consumers are also willing to pay a premium price for those food products. In particular, female consumers with diet-health knowledge (Øvrum et al., 2012) and consumers with chronic diseases (Marette et al., 2010) seem to be the groups who are both willing to pay higher prices for dairy products with health-enhancing features, and to take nutrition and health claims into account in their food decisions process (Marette et al., 2010; Øvrum et al., 2012). However, some evidence from low/medium quality studies indicates that the presence of nutrition claim generates negative effects on consumers' perceived pleasantness from the consumption of reduced fat dairy products (Kähkönen \& Tuorila, 1999, Johansen et al., 2011), effectiveness which is mitigated in health-conscious consumers committed to healthy eating habits, and less demanding about food taste (Johansen et al., 2011).

Health claims guarantee different levels of health efficacy and convey different health benefits (e.g. cholesterol reducing effects, support of the immune system, and support of bone health) (Bimbo et al., 2016). A high (van Kleef et al., 2005a) and a medium quality (Williams et al., 2008) study suggest that, among the many claims available in the marketplace, consumers prefer overall health claims to nutrition claims, and risk disease reduction claims to general function ones. Interest in risk reduction claims is found in highly educated consumers, often females, who have been directly or indirectly exposed to diseases, in consumers with a high level of diet-health related knowledge (Williams et al., 2008; Ares 
et al., 2010b), and in those using nutritional supplements (Hailu et al., 2009). Similar findings were reported by Annunziata \& Vecchio (2013) in their high quality study. These authors identified a consumer cluster composed mainly of highly educated females with children under 12 years of age, and of consumers adopting healthy diets, who preferred dairy products with risk reduction claims rather than other claims; the other cluster of respondents in their sample preferred generic claims related to the enhancement of general well-being (Annunziata \& Vecchio, 2013).

The results presented above do not depict clear patterns in consumers' acceptance for nutrition and health claims available in the market place. Results seem to vary according to how relevant a specific nutritional/health claim is, for the group of consumers examined. However, many high quality studies point to woman with diet-health knowledge, individuals with chronic diseases, and highly educated consumers, as those consumers groups which are more likely to take into account nutrition and health claims in their food choices, as well as to pay higher price for health-enhanced dairy versions. Additionally, consumers interested in dairy products with health claims may have a higher ability to understand them and to process the information conveyed by the health claims (Nocella \& Kennedy, 2012). Furthermore, claims are often formulated in complicated terms: shorter, easier to understand claims, may increase the acceptance of functional dairy products and facilitate the recovery of the high investment costs undertaken to develop and to market them (Siegrist et al., 2008).

With regard to brand, Deliza \& MacFie (1996) identify it as one of the most important extrinsic attributes influencing consumers' purchasing decisions for food products. Brands can signal quality and the manufacturer's guarantee of the truthfulness of what is declared on the package (Deliza \& MacFie, 1996). Similar findings emerge from studies investigating consumer acceptance and preference for nutrition-modified and functional dairy products (Ares et al., 2010a; Ares \& Deliza, 2010; Barrena \& Sanchez, 2010; Annunziata \& Vecchio, 2013).

The high quality study by Messina et al. (2008) showed that the influence of brand on older consumers' choices differs across countries, as older consumers from South America and Southern Europe are influenced more than those from other countries. Among medium quality studies, Ares et al. (2010a) found that brand affects willingness to purchase functional milk dessert, while Ares et al. (2010b) found that brand was the second attribute for magnitude, after carrier, to affect consumer choice of functional yogurts, and that the impact is as high as carrier, in affecting consumer's preferences among middle aged females.

Similar results emerge from the high quality study performed by Annunziata \& Vecchio (2013), where brand affects the choice of probiotic yogurts among a segment of young Italians with an average level of education, lower probability to engage in healthy eating habits, and low consumption frequency of probiotic dairy yogurt (Annunziata \& Vecchio, 2013); the same study also finds that brand's effect in shaping consumers' choices increases with consumers' familiarity with the brand, while brands do not affect food decisions in 
consumers with interest in health (Annunziata \& Vecchio, 2013), confirming findings of other studies (Barrios et al., 2007; Ares et al., 2010b).

Results of medium and high-quality studies supporting the notion that the brand positively affects consumers' attitudes and preference toward health-enhancing dairy products, were also found in two of the low-quality studies reviewed (Barrena \& Sanchez, 2010; Ares \& Deliza, 2010). Barrena \& Sanchez (2010) found that brand familiarity is one of the product's characteristics evaluated by households during their decision process to purchase probiotic milk (Barrena \& Sanchez, 2010), while Ares \& Deliza (2010) pointed out that brand was one of the most frequently mentioned item, after flavor, color and shape of the package among nutrition-modified milk desserts' packages features influencing purchases (Ares \& Deliza, 2010).

The findings presented above show a general consensus among the literature reviewed that brand increases the acceptance and motivates consumers' choice of nutrition-modified and functional dairy products over conventional ones. Such influence is particularly strong among consumers who are less likely to engage in a healthy lifestyle, while they have little to no effect on the choices of consumers with high interest in health. However, these results may be confounded by country-specific differences in education, in the proportion of individuals engaging in healthy lifestyles, and in the development stage of the health-enhancing foods' market. Last, packaging is another extrinsic product characteristic that attracts consumers' attention and can influence their purchasing decisions of health-enhancing dairy products. Among the studies identified, we found little emphasis on this factor. Ares \& Deliza (2010) explored the effect of packaging's attributes on consumer willingness to purchase nutritionmodified chocolate milk desserts. They found that the color and shape of packaging influence consumers' purchasing decisions and that brown packaging increases consumers' purchasing intentions for such dessert. Packaging shape, instead, shows mixed effects on consumers' intention to purchase a low-fat dessert, depending upon the expectations regarding the product's texture that the package shape generates in the consumers' minds (Ares \& Deliza, 2010). In summary, Ares \& Deliza's (2010) study proves that package's features affect consumers' acceptance and purchasing decisions, however more research is needed on this topic to corroborate the results of this study.

\section{Discussion, limitations and future research}

A systematic literature review technique was used to collect and consolidate the existing knowledge on consumers' acceptance and preferences toward nutrition-modified and functional dairy products. The quality of the studies identified was assessed by means of an ad hoc tool, and the studies' findings organized to give an overview of major factors influencing consumer behavior toward these products.

Overall, the findings of our systematic review support the existence of clear patterns characterizing consumers' acceptance and preferences for nutrition-modified and functional dairy products, differently than previous systematic reviews including studies covering 
different product categories (Ozen et al., 2012; Ozen et al., 2014) and in line with other reviews on consumer acceptance and preferences for health-enhancing food products (e.g. Frewer's et al. 2003; Siro's et al. 2008; and Lähteenmäki, 2013).

Our results confirm that gender and age play an important role in explaining different patterns of acceptance in relation to identified combinations of carriers and ingredients. Female consumers are more willing to use, and to include in their diet yogurt enriched with calcium, fiber and probiotics as well as consuming low-fat dairy products. Willingness to use/purchase functional and nutrition-modified dairy products increases with age, as older consumers perceive higher rewards from consuming such food versions, and show more interest in health. Therefore, female and older consumers characterize the groups of consumers likely to be most receptive to nutrition-modified and functional dairy products; as elderly people are the main users of resources within healthcare systems, and through promoting the consumption of dairy products with health enhanced features may improve their health and may have a beneficial impact on reducing national health care expenditure.

Our findings also support that diet-health and nutritional knowledge contribute to explain consumers' acceptance of nutrition-modified and functional dairy products; however, more research is needed in this area as most of the studies identified have used non-validated scales to assess this relationship. Consumer psychological traits also contribute to shape consumers' acceptance and preferences for nutrition-modified and functional dairy products. Among intrinsic product attributes, carriers appear as the most effective in influencing consumers' perceived healthiness; their effect is positive when a "natural" match between the carrier and the bioactive ingredient exists, and negative for "unnatural" matches, such as omega-3 added to yogurt. More research is needed on the role of different nutrition and health claims, as the existing literature provides conflicting results which may largely depend on the relevance of the nutrition or the health claim surveyed for the sample selected.

A novel result of this systematic review is that extrinsic product's characteristics such as a product's brand, and its package's features affect strongly consumers' choices for nutritionmodified and functional dairy products. Some of the studies included in this review pointed at brand as being the second most important product attribute, after the carrier, affecting consumers' evaluation of yogurt added with fiber and antioxidant. Also, brand recognition drives consumers' choice of yogurt with general functional claims among middle age Italian females with a sedentary lifestyle, and among Spanish households with children. Instead, a product's brand does not play a role when consumers chose dairy products with riskreduction health claims. Further, we identified one study exploring consumers' preferences for package's characteristics of nutrition-modified food products, which found that the package's features, such as its color, shape the consumer's preferences for low fat milk dessert shape consumers' expectations about the food product.

These novel findings may provide beneficial for manufacturers of nutrition-modified and functional dairy products, as they suggest the need to invest in building brand reputation to ensure market success. However, as consumers' interest in disease risk reduction claims does 
not seem affected by brand familiarity, firms investing in risk reduction claims may find it more profitable to focus their efforts in claim-developing activities, rather than in brand advertising. Furthermore, the success of nutrition-modified and functional products may be facilitated by marketing activities focusing on creating food packages which attract the consumers' attention and interest for such products.

Our study has three main limitations. First, our findings apply only to the acceptance and preferences for dairy products, and, as such, our analysis is limited in scope. Future research should focus on assessing consumer's behavior for others food categories, as well as the interrelationships between cross categories choices, which does not seem to have been explored so far.

Second, even though we are aware that taste stimuli play a pivotal role in food choices, and that a functionality/nutrition-modified feature provides added value to consumers as long as it doesn't modify the sensory properties of a food product original food (Verbeke, 2005; BechLarsen and Scholderer, 2007), we excluded this bulk of literature from this study as it is too vast and it deserves its own analysis. Thus, future research should account for the role played by taste stimuli on consumer's choices of functional/nutrition-modified food products.

Third, even though we aimed to provide a comprehensive picture of the many drivers affecting consumer acceptance and preferences for nutrition-modified and functional dairy products, the majority of the findings analyzed came from studies performed in Northern European countries, with few from Southern European and American countries. Therefore, in order to understand cultural, psychological and other aspects of purchasing behavior in other national contexts, more research needs to be conducted in Mediterranean, American and Asian countries. Expanding the pool of countries subject of analysis can allow food companies to reach international audiences more effectively.

The findings of this review also open to the possibility of new avenues of research. In the first place, some of our findings indicate that brand affects consumer's food choices when it is associated with nutrition and health claim. Future research should explore the possibility that consumers' acceptance and preferences for a product's feature may vary conditionally on the support provided by different brands. As it is well-known that a brand name may act as an additional guarantee of a label's truthfulness, more research on the interaction of different types of brand names and consumers' attitude towards specific features may provide beneficial for food manufacturers.

Last, it should be mentioned that none of the studies reviewed was conducted using methods aimed to improve the realism of choice experiments, such as virtual reality-based methods. As those methods are meant to increase their external validity (Van Herpen et al., 2016) they lend for their results to be more comparable across studies, which was one of the hurdles we faced in this systematic review and which is, in general, due to the fact that survey-based methods show high heterogeneity in study design (Van Kleef et al., 2005b). Future research should consider exploiting these new tools to corroborate the findings of survey-based 
research and, when a numerous enough body of research is available, to validate the findings of this review.

\section{Acknowledgments}

Authors are grateful to founders of project PON 01_00851 "Bioinnovation for high healthy value dairy production".

\section{References}

Almli, V.L., Næs, T., Enderli, G., Sulmont-Rossé, C., Issanchou, S. \& Hersleth, M. (2011). Consumers' acceptance of innovations in traditional cheese. A comparative study in France and Norway. Appetite, 57, 110-120.

Alevizos, A., Mihas, C., \& Mariolis, A. (2007). Advertising campaigns of sterol enriched food. An often neglected cause of reduced compliance to lipid lowering drug therapy. Cardiovascular Drugs and Therapy, 21(2), 133-134.

Annunziata, A., \& Vecchio, R. (2013). Consumer perception of functional foods: A conjoint analysis with probiotics. Food Quality and Preference, 28, 348-355.

Ares, G., \& Deliza, R. (2010). Identifying important package features of milk desserts using free listing and word association. Food Quality and Preference, 21, 621-628.

Ares, G., \& Gámbaro, A. (2007). Influence of gender, age and motives underlying food choice on perceived healthiness and willingness to try functional foods. Appetite, 49, 148 158.

Ares, G., Besio, M., Giménez, A., \& Deliza, R. (2010a). Relationship between involvement and functional milk desserts intention to purchase. Influence on attitude towards packaging characteristics. Appetite, 55, 298-304.

Ares, G., Giménez, A., \& Deliza, R. (2010b). Influence of three non-sensory factors on consumer choice of functional yogurts over regular ones. Food Quality and Preference, 21, 361-367.

Ares, G., Giménez, A., \& Gámbaro, A. (2009). Consumer perceived healthiness and willingness to try functional milk desserts. Influence of ingredient, ingredient name and health claim. Food Quality and Preference, 20, 50-56.

Ares, G., Giménez, A., \& Gámbaro, A. (2008). Influence of nutritional knowledge on perceived healthiness and willingness to try functional foods. Appetite, 51, 663-668.

Barrena, R., \& Sánchez, M. (2010). The link between household structure and the level of abstraction in the purchase decision process: an analysis using a functional food. Agribusiness, 26, 243-264.

Barrios, E.X., Bayarri, S., Carbonell, I., Izquierdo, L., \& Costell, E. (2008). Consumer Attitudes and Opinions Toward Functional Foods: A Focus Group Study. Journal of Sensory Studies, 23, 514-525. 
Bech-Larsen, T., \& Grunert, K.G. (2003). The perceived healthiness of functional foods: A conjoint study of Danish, Finnish and American consumers' perception of functional foods. Appetite, 40, 9-14.

Bech-Larsen, T., \& Scholderer, J. (2007). Functional foods in Europe: consumer research, market experiences and regulatory aspects. Trends in Food Science \& Technology, 18(4), 231-234.

Bigliardi, B., \& Galati, F. (2013). Innovation trends in the food industry: The case of functional foods. Trends in Food Science \& Technology, 31, 118-129.

Bimbo, F., Bonanno, A., \& Viscecchia, R. (2016). Do health claims add value? The role of functionality, effectiveness and brand. European Review of Agricultural Economics, 43(5), 761-780.

Bleiel, J. (2010). Functional foods from the perspective of the consumer: How to make it a success?. International dairy journal, 20(4) 303-306.

Bonanno, A. (2012). Some Like It Healthy: Demand for Functional and Conventional Yogurts in the Italian Market. Agribusiness, 28, 67-85.

Bower, J. A., Saadat, M. A., \& Whitten, C. (2003). Effect of liking, information and consumer characteristics on purchase intention and willingness to pay more for a fat spread with a proven health benefit. Food Quality and Preference, 14, 65-74.

Chase, D., Emunu, J.P., Nilsson, T., McCann-Hiltz,D., \& Peng, Y. (2009). Canadian Consumers' Purchasing Behavior of Omega-3 Products. Journal of Food Distribution Research, 40(2), 12-23.

Cooper, H. M. (1998). Synthesizing research: A guide for literature reviews: Vol. 2. Applied social research methods (3rd ed.). Thousand Oaks, CA: Sage.

Cox, D.N., Evans, G., \& Lease, H.J. (2007). Predictors of Australian consumers' intentions to consume conventional and novel sources of long-chain omega-3 fatty acids. Public Health Nutrition, 11(1), 8-16.

Cox, D.N., Evans, G., \& Lease, H.J. (2011). The influence of product attributes, consumer attitudes and characteristics on the acceptance of: (1) Novel bread and milk, and dietary supplements and (2) fish and novel meats as dietary vehicles of long chain omega 3 fatty acids. Food Quality and Preference, 22, 205-212.

Cox, D. N., Hendrie, G. A., \& Carty, D. (2015). Sensitivity, hedonics and preferences for basic tastes and fat amongst adults and children of differing weight status: A comprehensive review. Food Quality and Preference, 41, 112-120.

de Jong, N., Ocké, M.C., Branderhorst, H.A.C. \& Friele, R. (2003). Demographic and lifestyle characteristics of functional food consumers and dietary supplement users. British Journal of Nutrition, 89, 273-281.

Deliza, R., \& MacFie, H.J.H. (1996). The generation of sensory expectation by external cues 
and its effect on sensory perception and hedonic ratings: A review. Journal of Sensory Studies, 11, 103-128.

Food and Drug Administration (FDA) (2013). Guidance for Industry: A Food Labeling Guide available http://www.fda.gov/Food/GuidanceRegulation/GuidanceDocumentsRegulatoryInformation/L abelingNutrition/ucm2006828.htm last access 26/10/2015.

Ford, N. (2011). The essential guide to using the web for research. Thousand Oaks, CA: Sage.

Frewer, L., Scholderer, J., \& Lambert, N. (2003). Consumer acceptance of functional foods: issues for the future. British Food Journal, 105(10), 714-731.

Grunert, K. G., Hartvig Larsen, H., Madsen, T. K., \& Baadsgaard, A. (1996). Market Orientation in Food and Agriculture. Norwell, MA: Kluwer.

Grunert, K. G., Bech-Larsen, T., \& Bredahl, L. (2000). Three issues in consumer quality perception and acceptance of dairy products. International Dairy Journal, 10(8), 575-584.

Hailu, G., Boecker, A., Henson, S., \& Cranfield, J. (2009). Consumer valuation of functional foods and nutraceuticals in Canada. A conjoint study using probiotics. Appetite, 52, 257-265.

Hardy, N. (2010). Future Innovations in Food and Drinks to 2015, Report No. BI00014-024, Business Insights, London.

Heasman, M., \& Mellentin, J. (2001). The Functional Foods Revolution: Healthy People, Healthy Profits?, Earthscan Publications Ltd, London.

Hendrie, G. A., Coveney, J., \& Cox, D. (2008). Exploring nutrition knowledge and the demographic variation in knowledge levels in an Australian community sample. Public Health Nutrition, 11(12), 1365-1371.

Joanna Briggs Institute. (2009). Comprehensive systematic review module 3: The systematic review of quantitative evidence.

Johansen, S.B., Næs, T., \& Hersleth, M. (2011). Motivation for choice and healthiness perception of calorie-reduced dairy products. A cross-cultural study. Appetite, 56, 15-24.

Kähkönen, P., \& Tuorila, H. (1999). Consumer responses to reduced and regular fat content in different products: effects of gender, involvement and health concern. Food Quality and Preference, 10, 83-91.

Khan, R.S., Grigor, J.V., Win A.G., \& Boland, M. (2014). Differentiating aspects of product innovation processes in the food industry. British Food Journal, 116(8), 1346-1368.

Krutulyte, R., Grunert, K.G., Scholderer, J., Hagemanna, K.S., Elgaarda, P., Nielsena, B., \&Graverholt, J.P. (2008). Motivational factors for consuming omega-3 PUFAs: An exploratory study with Danish consumers. Appetite, 5(1), 137-147. 
Krutulyte, R., Grunert, K. G., Scholderer, J., Lähteenmäki, L., Hagemann, K. S., Elgaard, P., Nielsen, B., \& Graverholt, J. P. (2011). Perceived fit of different combinations of carriers and functional ingredients and its effect on purchase intention. Food Quality and Preference, 22(1), 11-16.

Labrecque, J., Doyon, M., Bellavance, F., \& Kolodinsky, J. (2006). Acceptance of Functional Foods: A Comparison of French, American, and French Canadian Consumers. Canadian Journal of Agricultural Economics/Revue canadienned'agroeconomie, 54, 647-661.

Landström, E., Hursti, U.-K.K., Becker, W., \& Magnusson, M. (2007). Use of functional foods among Swedish consumers is related to health-consciousness and perceived effect. British Journal of Nutrition, 98, 1058-1069.

Landström, E., Hursti, U.-K.K., \& Magnusson, M. (2009). Functional foods compensate for an unhealthy lifestyle. Some Swedish consumers' impressions and perceived need of functional foods. Appetite, 53, 34-43.

Lähteenmäki, L., Lampila, P., Grunert, K., Boztug, Y., Ueland, Ø., Åström, A., \& Martinsdóttir, E. (2010). Impact of health-related claims on the perception of other product attributes. Food Policy, 35(3), 230-239.

Lähteenmäki, L. (2013). Claiming health in food products. Food Quality and Preference, 27(2), 196-201.

Littell, J.H., \& College, B.M. (2006). Systematic Reviews in the Social Sciences: A Review. Evidence \& Policy, 2(4), 535-537.

Littell, J.H., Corcoran, J., \& Pillai, V. (2008). Syematic Reviews and Meta-Analysis. Oxford University Press.

Maynard, L.J. (2005). Value-Added Pros and Cons: Can Producers Profit from High-CLA Milk and Dairy Products?. Advances in Dairy Technology, 17, 49-60.

Marette, S., Roosen, J., Blanchemanche, S., \& Feinblatt-Mélèze, E. (2010). Functional food, uncertainty and consumers' choices: A lab experiment with enriched yoghurts for lowering cholesterol. Food Policy, 35, 419-428.

Marketsand Markets (2015). Functional Food Ingredients Market worth \$2.5 Billion by 2020 . available at Markets and Markets http://www.marketsandmarkets.com/PressReleases/functional-food-ingredients.asp last access 27/12/2015.

Messina, F., Saba, A., Turrini, A., Raats, M., Lumbers, M., \& Team, F. in L.L., (2008). Older people's perceptions towards conventional and functional yoghurts through the repertory grid method: A cross-country study. British Food Journal, 110, 790-804.

Mullie, P., Godderis, L., \& Clarys, P. (2012). Determinants and nutritional implications associated with low-fat food consumption. Appetite, 58, 34-38.

Niva, M. (2006). Can we predict who adopts health-promoting foods? Users of functional 
foods in Finland. Scandinavian Journal of Food and Nutrition, 50, 13-24.

Nocella, G., \& Kennedy, O. (2012) Food health claims - What consumers understand. Food Policy, 37 (5), 571-580.

Nutraingredients (2009). US leads in healthy food product launches. Available at http://www.nutraingredients-usa.com/Markets/US-leads-in-healthy-food-product-launches

Retrieved 09.11.2016.

O’Brien, G.M., Stewart-Knox, B.J., McKinley, A., de Almeida, M.D.V., \& Gibney, M.J. (2012). Perceived risk of metabolic syndrome and attitudes towards fat-modified food concepts among European consumers. Food Quality and Preference, 23, 79-85.

Øvrum, A., Alfnes, F., Almli, V.L., \& Rickertsen, K. (2012). Health information and diet choices: Results from a cheese experiment. Food Policy, 37, 520-529.

Özen, A. E., Pons, A., \& Tur, J.A. (2012). Worldwide consumption of functional foods: a systematic review. Nutrition Reviews, 70, 472-481.

Özen, A. E., Bibiloni, M., Pons, A., \& Tur, J. A. (2014). Consumption of functional foods in Europe; a systematic review. Nutricion Hospitalaria, 29(3), 470-478

Ozer, B.H., \& Kirmaci, H.A. (2010). Functional milks and dairy beverages. International Journal of Dairy Technology, 63, 1-15.

Parmenter, K., \& Wardle, J. (1999). Development of a general Nutrition Knowledge Questionnaire for adults. European Journal of Clinical Nutrition, 53, 293-303.

Peng, Y., West, G.E., \& Wang, C. (2006). Consumer Attitudes and Acceptance of CLA - Enriched Dairy Products. Canadian Journal of Agricultural Economics/Revue canadienned'agroeconomie, 54, 663-684.

Playne, M.J., Bennett, L.E., \& Smithers, G.W. (2003) Functional dairy foods and ingredients. Austalian Journal Dairy Technology, 58, 242-264.

Pliner, P., \& Hobden, K. (1992). Development of a scale to measure the trait of food neophobia in humans. Appetite, 19, 105-120.

Poulsen, C. S., Juhl, H. J., Kristensen, K., Bech, A. C., \& Engelund, E. (1996). Quality guidance and quality formation. Food Quality and Preference, 7, 127-135.

Regulation of European Commission (EU) No 1924/2006 of the European Parliament and of the Council of 20 December 2006 on nutrition and health claims made on foods.

Regulation of European Commission (EU) No 432/2012 of 16 May 2012. Establishing a list of permitted health claims made on foods, other than those referring to the reduction of disease risk and to children's development and health.

Research and Markets (2014). Global Functional Food and Nutraceuticals Market (2014 2020) - By Type (Foods, Beverages, Supplements); Benefits (Health and Wellness, Disease Prevention, Fitness, Beauty); Origin \& Ingredient. 
Rogers, R.W. (1975). A Protection Motivation Theory of Fear Appeals and Attitude Change. The Journal of Psychology: Interdisciplinary and Applied, 91 (1), 93-114.

Roininen, K., Lähteenmäki, L., \& Tuorila H. (1999). Quantification of consumer attitudes to health and hedonic characteristics of foods. Appetite, 33, 71-88.

Siegrist, M., Stampfli, N., \& Kastenholz, H. (2008). Consumers' willingness to buy functional foods. The influence of carrier, benefit and trust. Appetite, 51, 526-529.

Siró, I., Kápolna, E., Kápolna, B., \& Lugasi, A., (2008). Functional food. Product development, marketing and consumer acceptance-A review. Appetite, 51, 456-467.

Starling, S., (2014). Functional foods resist recession but failure rate stays high: Analyst. July $7^{\text {th }}$. Available at Nutraingredients.com http://www.nutraingredients.com/ConsumerTrends/Functional-foods-resist-recession-but-failure-rate-stays-high-Analyst Retrieved 09.04.2015.

Stein, A.J., \& Rodríguez-Cerezo, E. (2008). Functional food in the European Union. Technical Report by the Joint Research Centre of the European Commission, EUR 23380 EN. Luxemburg: European Communities.

Urala, N., \& Lähteenmäki, L. (2004). Attitudes behind consumers' willingness to use functional foods. Food Quality and Preference, 15, 793-803.

Urala, N., \& Lähteenmäki, L. (2007). Consumers' changing attitudes towards functional foods. Food Quality and Preference, 18, 1-12.

Valls, J, Pasamontes, N., Pantaleón, A., Vinaixa, S., Vaqué, M., Soler, A., Millán, S., \& Gómez, X., 2013. Prospects of Functional Foods/ Nutraceuticals and Markets. Natural Products, Springer-Verlag Berlin Heidelberg.

Van Kleef, E., van Trijp, H., Luning, P., \& Jongen, W. M. (2002). Consumer-oriented functional food development: how well do functional disciplines reflect the 'voice of the consumer'?. Trends in Food Science \& Technology, 13(3), 93-101.

Van Kleef, E., van Trijp, H.C.M., \& Luning, P. (2005a). Functional foods: health claim-food product compatibility and the impact of health claim framing on consumer evaluation. Appetite, 44, 299-308.

Van Kleef, E., van Trijp, H. C., \& Luning, P. (2005b). Consumer research in the early stages of new product development: a critical review of methods and techniques. Food quality and preference, 16(3), 181-201.

van Herpen, E., van den Broek, E., van Trijp, H. C., \& Yu, T. (2016). Can a virtual supermarket bring realism into the lab? Comparing shopping behavior using virtual and pictorial store representations to behavior in a physical store. Appetite, 107, 196-207.

Verbeke, W. (2005). Consumer acceptance of functional foods: socio-demographic, cognitive and attitudinal determinants. Food Quality and Preference, 16, 45-57. 
Viana, J. V., Da Cruz, A. G., Zoellner, S. S., Silva, R., \& Batista, A. L. (2008). Probiotic foods: consumer perception and attitudes. International journal of food science \& technology, 43(9), 1577 - 1580.

Wahba, S. A., Arrafa, A. M., Saleh, N. A., Mekkawy, A. A., \& Ahmed, R. T. (2006). Knowledge, attitudes toward functional foods among adults working in the national research center. Journal of Applied Sciences Research, 2(1), 39-43.

Wardle, J., Haase, A. M., Steptoe, A., Nillapun, M., Jonwutiwes, K., \& Bellisie, F. (2004). Gender differences in food choice: the contribution of health beliefs and dieting. Annals of Behavioral Medicine, 27(2), 107-116.

Williams, P., Ridges, L., Batterham, M., Ripper, B., \& Hung, M.C. (2008). Australian consumer attitudes to health claim - food product compatibility for functional foods. Food Policy, 33, 640-643.

Zandstra, E., de Graaf, C., \& Van Staveren, W., (2001). Influence of health and taste attitudes on consumption of low- and high-fat foods. Food Quality and Preference, 12, 75-85. 
Chapter 2

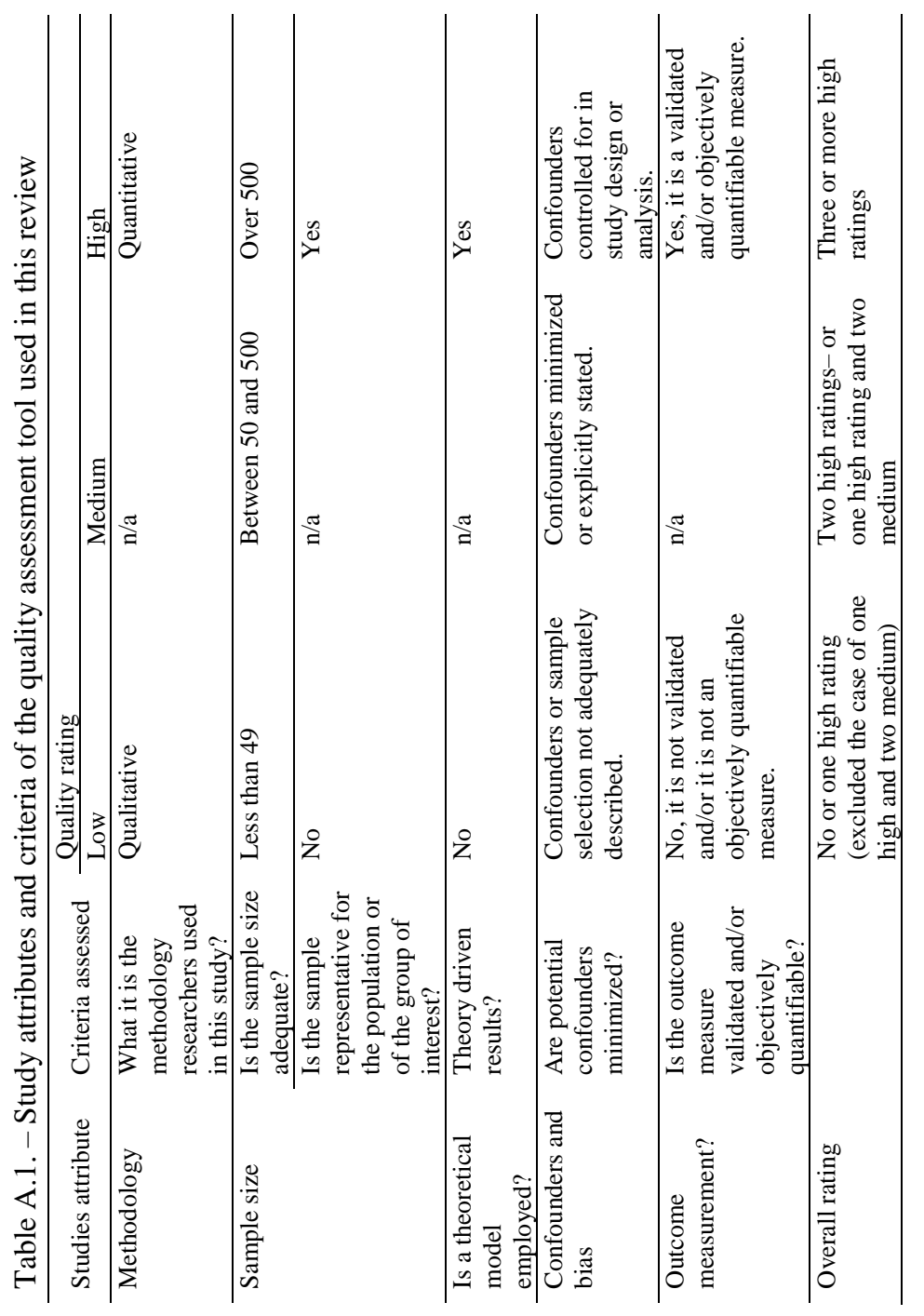


Chapter 2

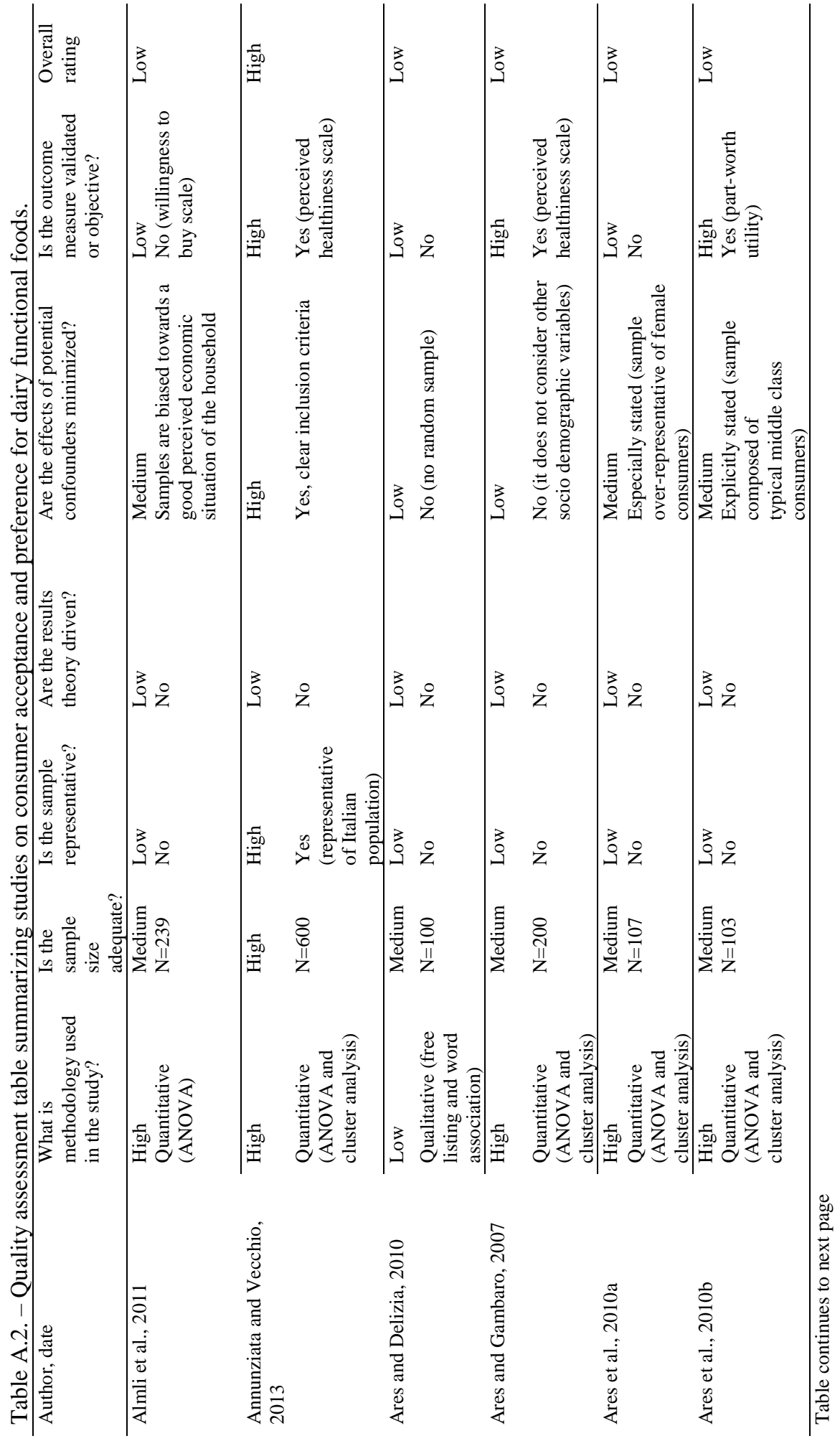


Chapter 2

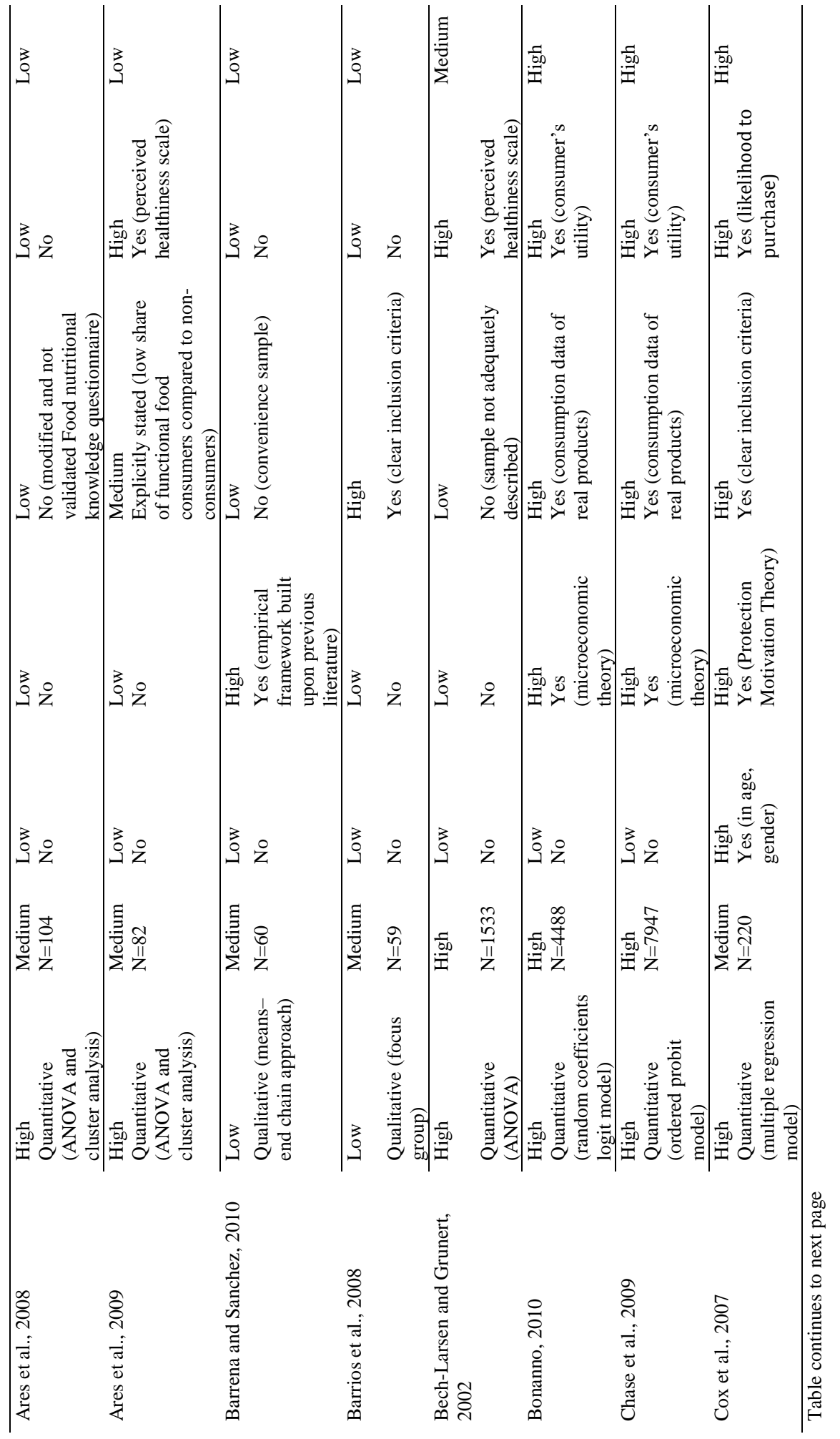


Chapter 2

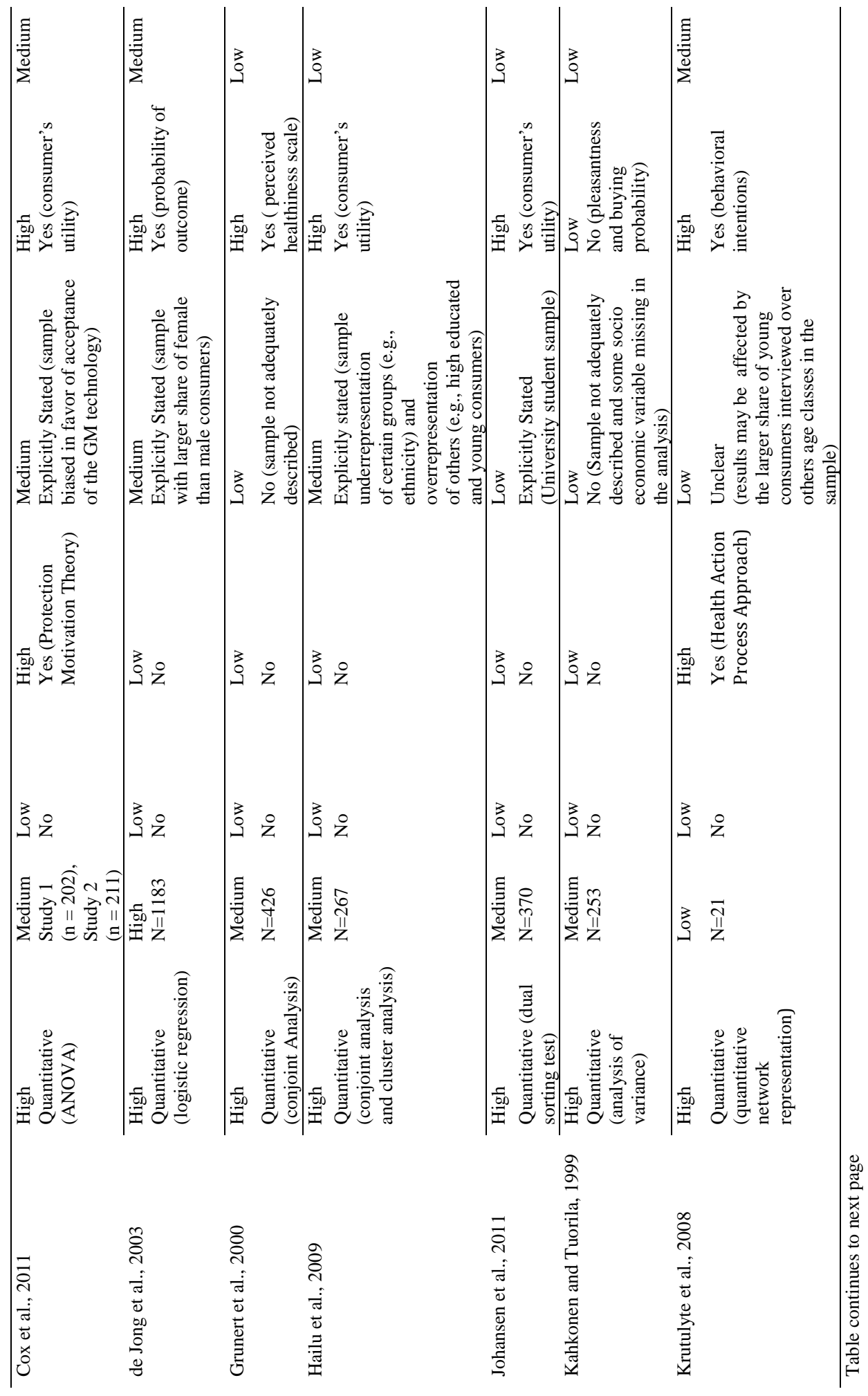


Chapter 2

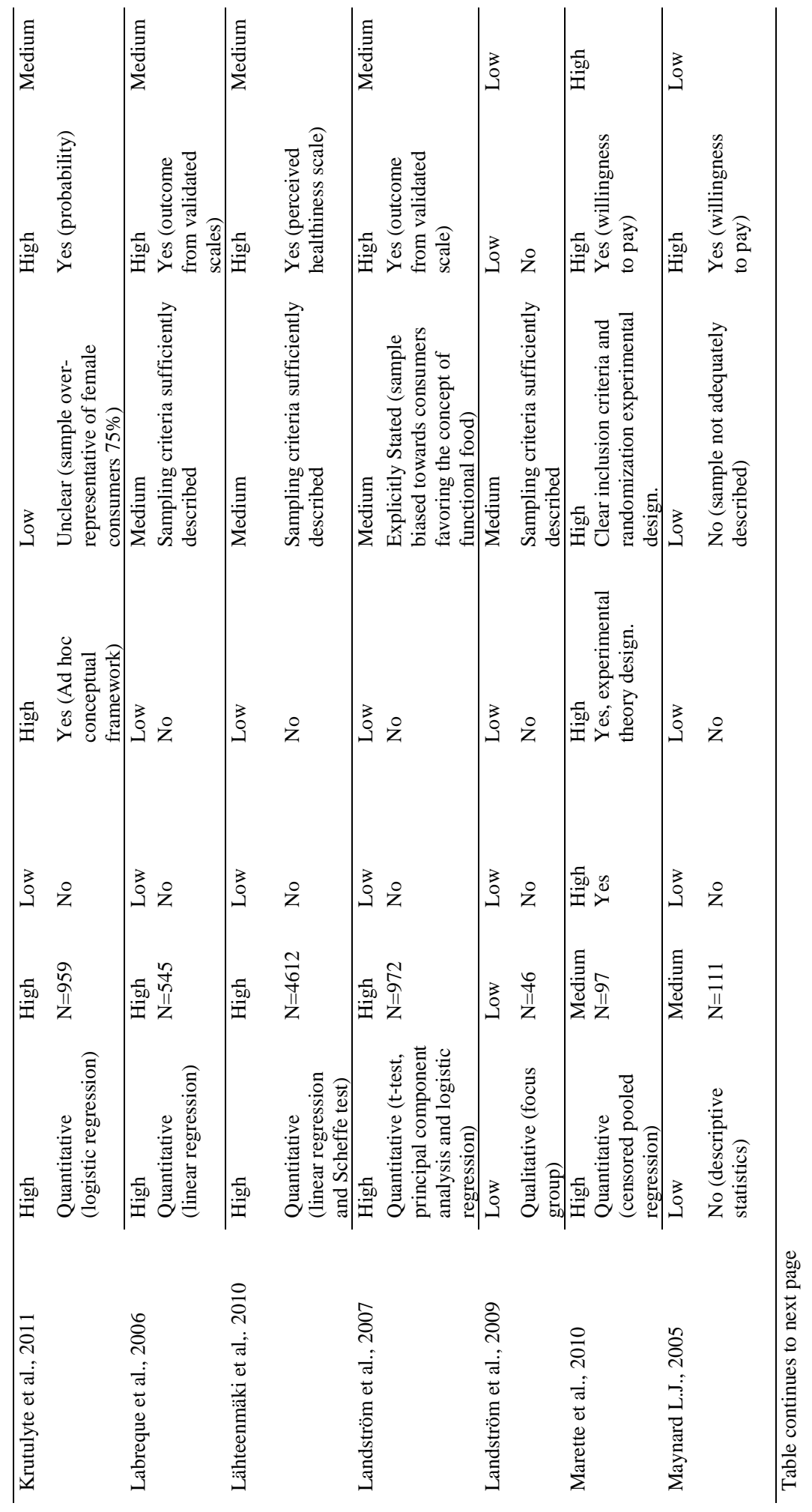


Chapter 2

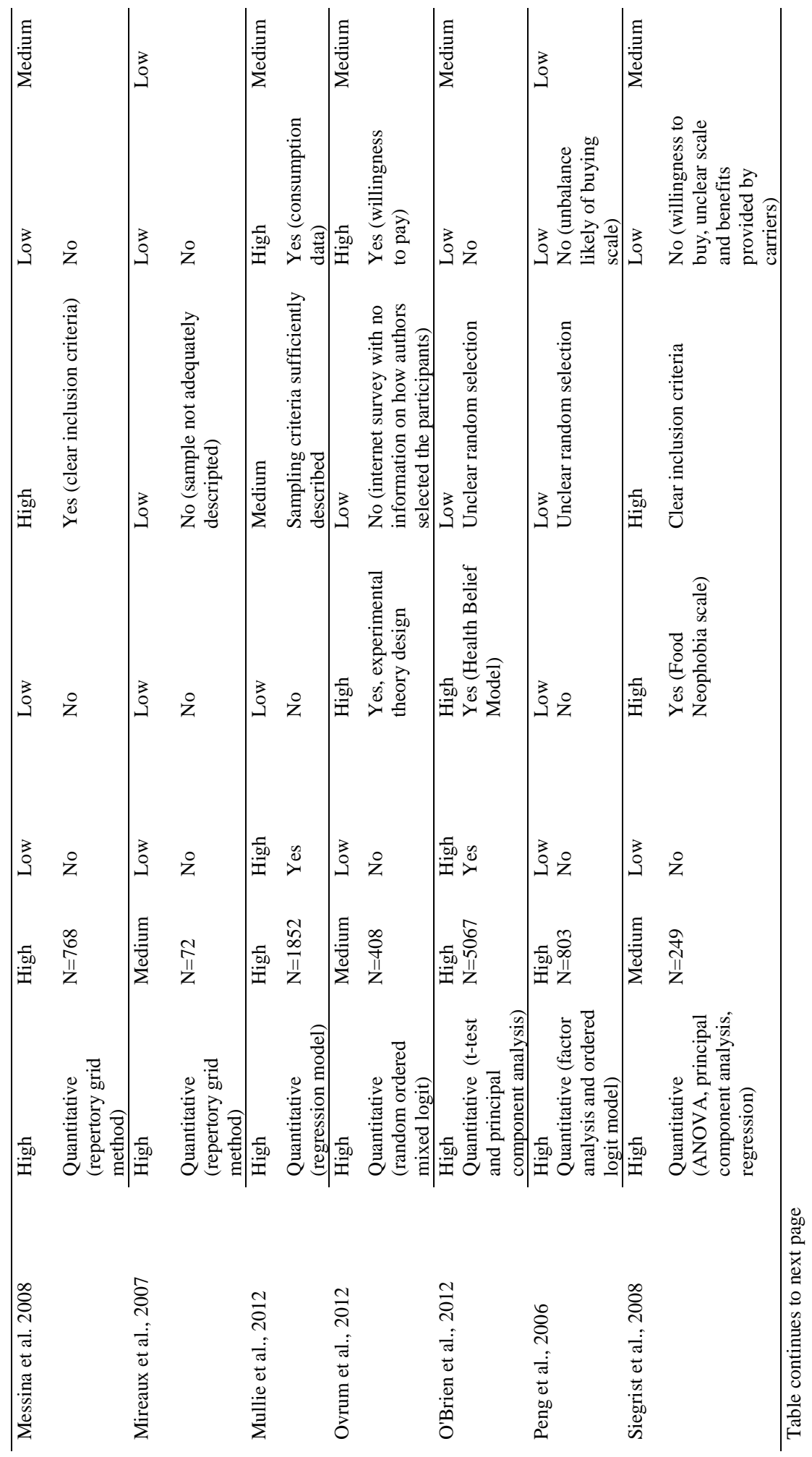


Chapter 2

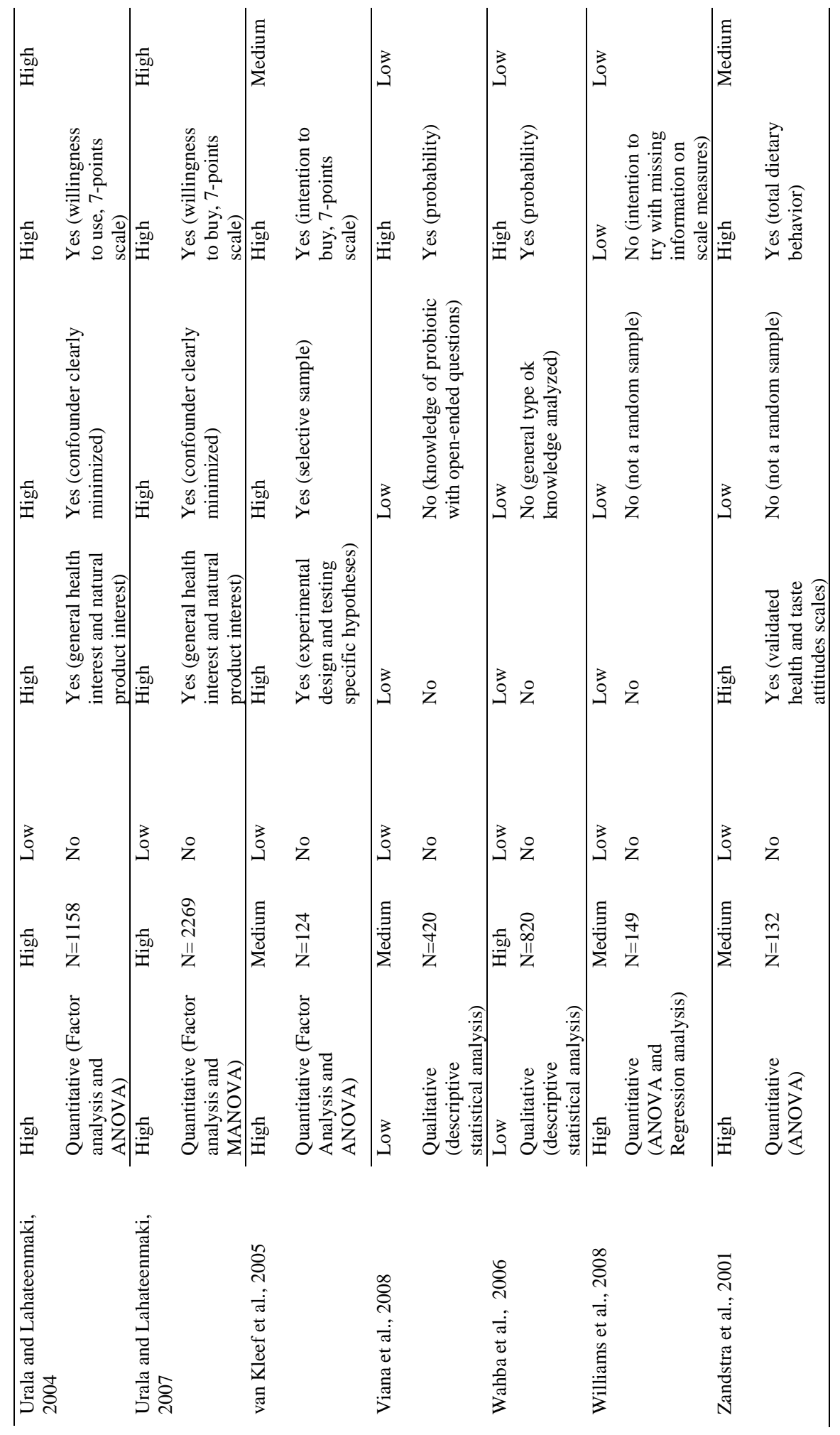




$$
-
$$

(3)

- 


\section{Chapter 3}

Do Health Claims' Add Value? The Role of Functionality, Effectiveness, and Brand.

Francesco Bimbo, Alessandro Bonanno, Rosaria Viscecchia. 
Do Health Claims' Add Value? The Role of Functionality, Effectiveness, and Brand.

\begin{abstract}
European functional food manufacturers experience high costs of production, marketing, and compliance with Regulation EC No. 1924/2006, requiring high premiums to recover these costs. We review the literature on consumers' acceptance of health-enhancing food products, and identify three factors potentially affecting health claims' premiums: health claims' type, efficacy, and brands. We estimate the contribution of these factors on health claims' implicit prices using a database of Italian yogurt sales and a hedonic price model. We find premiums differing by claim type; that "risk diseases reduction" claims outperforming "general function" claims; and that the support of familiar brands affects health claims' premiums.
\end{abstract}

Keywords: functional food, health claims, hedonic price model

\title{
1. Introduction
}

The market for health-enhancing food products has been growing largely since these products appeared on the market in the mid-90s (Menrad, 2003). In 2008, the global market for functional foods ${ }^{1}$ represented the fastest growing food market worldwide, with an annual growth rate of $10 \%$ (Barreiro-Hurle et al., 2008) and it was sized at USD 24.2 billion in 2011(Valls et al., 2013). Europe, Japan and U.S., together capture more than $90 \%$ of functional food sales and represent target markets for many producers (Valls et al., 2013).

In spite of the promising market trends, European food manufacturers producing products with health claims face considerable hurdles. In the first place, the costs of developing and marketing functional products are higher than those of conventional ones (Menrad, 2003; Sirò et al., 2008; Brookes, 2010). ${ }^{2}$ Second, functional products have a high failure rate: some evidence exists that at least 75 per cent of newly launched functional food products are withdrawn from the market, as they are priced too high compared to conventional versions (Stein and Rodríguez-Cerezo, 2008). ${ }^{3}$ Third, as the potential to deliver a health benefit is a credence attribute (Grunert, 2005a), asymmetric information may be present in the functional

\footnotetext{
${ }^{1}$ EU Regulation (EC) No. 1924/2006 employs as working definition of functional product that proposed by Diplock et al.in 1999, which defines functional food as any "[...] modified foods or food ingredients which may provide a health benefit beyond nutrients they contain". .

${ }^{2}$ For example, Menrad (2003) collected experts' estimates for the cost needed to develop and market Nestlés LC1 yogurt and Becel ${ }^{\circledR}$ proactive margarine. Such estimates exceeded 50 million USD for each product, much higher than investments needed to develop and market new conventional new products, estimated not to exceed 1 or 2 million USD (Sirò et al., 2008).

${ }^{3}$ Price may not be the only hurdle resulting in functional foods market failure. Other issues may be related to the different levels of consumers' acceptance for products carrying health claims showing different levels of complexity and different framing (Grunert et al., 2009). Also a product's inability to convey the claimed benefits to a specific target audience (i.e. the relevance of a health claim) may be a crucial determinant of product failure; for example, evidence exists that consumers who have directly, or via family members, suffered health condition, are more favorable towards products with health claims targeting the conditions / symptoms they have suffered (Van Kleef, Van Trijp and Luning, 2005a). Creating a refined marketing strategy to target such narrow consumer group may be problematic, and the implementation of broader marketing efforts may result in large expenses without significant returns.
} 
food market (Bonanno, Huang and Liu, 2015). In order to reduce asymmetric information ${ }^{4}$ and to protect consumers from the potential presence of untruthful health claims, the European Union introduced Regulation (EC) No.1924/2006. To achieve its goals, EU Regulation (EC) No 1924/2006 imposes additional burdens to manufacturers operating in the European market.

Regulation (EC) No. 1924/2006 sets the criteria for food manufacturers wanting to use health claims on their products' label. According to the Regulation (EC) No. 1924/2006, health claims are mainly classified in two categories: "disease risk reduction" claims (Article14 claims), and "general function" claims (article 13 claims). ${ }^{5}$ All claims need to be approved by the European Food Safety Authority (EFSA). While the approval of Article 13 claims is based upon existing knowledge regarding the relationship between a nutrient or active compound and its effect on human health, Article 14 claims must go through a rigorous authorization procedure based on a case-by-case dossier review by EFSA's panel on Dietetic Products, Nutrition and Allergies (NDA). The dossiers submitted must include scientific evidence supporting a causal relationship between the active principle and the claimed effect.

Compliance with Regulation (EC) No.1924/2006 is costly and fairly stringent, if compared to others regulatory system, such as the American and Canadian ones (Hobbs et al., 2014). Brookes (2010) assesses the economic impact of European Regulation on food supplement manufacturers' operation finding that, in the case of "general function" claims, the estimated average cost to develop and market products carrying such claims ranges from EUR 980 to EUR 1,663, while the cost of a "reduction disease risk" claim is more than tenfold. Also, the costs associated with clinical trials needed to produce scientific evidence supporting article 14 claims applications may result in an additional cost of EUR 0.25 million to EUR 1 million (Brookes, 2010). ${ }^{6}$

Given the hurdles discussed above, research on the performance of products carrying health claims seems necessary to support manufacturers' decision-making. Firms' ability to recover the high investments necessary for taking these types of products to market, depends upon the value that the market attaches to health claims. Evidence of consumer's willingness to pay a premium price for health-enhancing features is mixed: while some authors find consumers having a higher willingness to pay for these products (Ding et al., 2012; Balcome et al., 2014; Moro et al., 2015), others indicate that only a limited price premium is achievable (Menrad, 2003; Siro' et al., 2008). Thus, assessing the premium price attached to a health claim may provide valuable information for food manufacturers who may experience low returns for the high investments undertaken to develop functional food products.

\footnotetext{
${ }^{4}$ As discusses elsewhere in the literature, in markets characterized by asymmetric information, labeling policies may circumvent this issue by making the information initially held by manufacturers also available to the consumers (Teisl and Roe, 1998; Malla, Hobbs and Perger, 2007).

${ }^{5}$ Claims falling under article 13 are further divided in two groups: 1) Article 13.1: "general function" claims related to growth, development, and functions of the body; 2) article 13.5 claims, pertaining to general function claims based on new and/or proprietary data. Article 14 refers to the reduction of disease risk or to children's development and health. Article 13.5 and Article 14 claims undergo to same authorization procedure, illustrated in the main text. Regulation (EC) No.1924/2006 also includes nutrition claims (Article 8), which are not considered in this analysis.

${ }^{6}$ Additionally, food manufacturers receiving a negative opinion /assessment by EFSA may incur in additional adjustment costs. Such costs are due to a firm's decision to modify a product and/or a dossier to overturn EFSA's negative decision. The estimated average cost for these actions, ranging from product reformulation to the improvement of the dossier supporting the application, is EUR 126,700 (Brookes, 2010).
} 
Surprisingly, only few analyses have attempted to assess the performance of products carrying health claims. Bonanno (2013) focuses on the extent of product differentiation in the Italian yogurt market with particular emphasis on the presence of functional products. Bonanno, Huang and Liu (2015), focusing on the same market, simulated welfare changes due to Regulation (EC) No. 1924/2006. Other analyses exist, estimating directly the premium price associated to some functional attributes / health claims in products available in the marketplace, however using limited geographical samples (e.g. Carlucci et al., 2013; Szathvary and Trestini, 2014) or without inferring on different types of claims (Bonanno, forthcoming).

In this paper, we assess the premium price attached to health claims, using the Italian yogurt market as a case study. In order to achieve this objective we use a hedonic price model and a large database of yogurt sales encompassing the entire Italian market. To establish what factors may affect the value of a health claim through their influence on consumers' preferences, we first review the literature on consumers' acceptance of functional product / products carrying health claims. We then specify and estimate a model to obtain the implicit price associated with health claims, decomposing it according to three of the main factors which the literature suggests to affect their acceptance: the type of health benefit provided, the guaranteed level of efficacy on human health, and whether a claim is supported by a familiar brand. Even though a small number of empirical analyses exist that assess the relevance of one or more of these three factors to the market valuation of health claims (e.g. Carlucci et al.2013; Szathvary and Trestini 2014; Bonanno, Huang and Liu 2015) no study exists where the three factors' contribution to prices is explored jointly.

We select the Italian yogurt market as a case study for three reasons. First, yogurt is considered intrinsically healthy and it is one of the most credible carriers of functional ingredients (Sirò et al., 2008; Ozer and Kirmaci, 2010). In fact, the category of fermented functional dairy accounts for nearly $43 \%$ of the total functional products' market (Ozer and Kirmaci, 2010). Second, Italy saw the launch of the highest number (592) of new healthy products among European Countries between 2005 and 2009; the majority of the new products introduced were functional yogurts benefiting digestive health (336), the immune system (99) and cardiovascular health (65) (Nutraingredients, 2009). Third, the Italian yogurt market is characterized by a high level of differentiation in terms of health attributes, with manufacturers offering a variety of products carrying different health claims (Bonanno, 2012, 2013) which appears suitable to analyze the problem at hand.

\section{Literature Review}

The functional foods' market has been subject of analysis for many marketing and consumer scholars over the last 10 years, resulting in an extensive body of literature on consumers' acceptance of functional foods and on the products' market success (inter alia, Van Kleff et al., 2005a; Ares and Gambaro, 2007; Siegrist et al., 2008; Ares et al., 2010b; Krutulyte et al., 
2008). ${ }^{7}$ Within this large body of literature, one can identify at least three broad groups of product features that can influence consumers' acceptance of functional foods: the type of health benefit provided (e.g. in the case of yogurts, cholesterol reduction, support for the immune system, promoting bowel regularity, bone health); the claimed effectiveness on human health (reduction disease risk vs. general function activity); and the support of a familiar brand.

First, scholars found that consumers' acceptance of functional products can depend upon the matching of specific functional ingredients with product carriers (Van Kleef et al., 2005a; Krutulyte et al., 2011). ${ }^{8}$ Products carrying a more "natural" ${ }^{\prime 9}$ ingredient-carrier combination are more likely to be accepted by consumers (Niva, 2006; Landström et al., 2009). For example, consumers show a positive attitude towards milk added with calcium and bread with fibres, since these combinations can be perceived as "natural" matches thanks to the functional component being inherent to the carrier (Niva, 2006; Krutulyte et al., 2011). Negative attitudes can be observed for yogurts added with omega-3 or fibres as the match between carrier and ingredient appears less natural than, for example, omega-3 with fishbased products, or fibre with bread (Krutulyte et al., 2008). By the same token, some evidence exists that familiar ingredients are preferred to unfamiliar ones (Grunert et al., 2009). Thus, within a product category, not all the types of functionality may be perceived positively, which hints to the possibility that, within the same category, different health claims will result in different premiums. In fact, Carlucci et al.(2013) using supermarket-level data in one Italian region, found a premium price for yogurts added with fibre, probiotic and calcium in a measure of $+32.33 \%,+24.45 \%$ and $+27.18 \%$, respectively.

Second, the level of efficacy guaranteed by a claim on human health, can influence consumers' acceptance of a functional product according to whether a product conveys a disease risk reduction, general function, or a nutrition claim (Verbeke et al., 2009). Evidence suggests that consumers prefer claims substantiated by scientific evidence and guaranteed by the government or third parties (Siegrist et al., 2008). Also, at least in the case of functional dairy products, consumers seem to prefer health claims rather than nutritional claims and, among the former, disease risk reduction over general function claims (van Kleef et al., 2005a; Hailu et al., 2009). The most preferred risk reduction claims on functional dairy products are those promising to reduce the risk of osteoporosis and cardiovascular diseases which seem well accepted by consumers of dairy foods (Siegrist et al., 2008; Ares et al., 2010b; Annunziata and Vecchio, 2013). Although empirical evidence using observed behaviour data supporting this point is limited, some evidence exists confirming that health claims outperform nutritional claims by leading to a higher premium price: Szathvary and

\footnotetext{
${ }^{7}$ Most analyses were performed using stated preferences data, where respondents (mainly consumers) are asked to state their attitudes, perceptions and intentions toward a product concept, usually by means of a Likert scale. Readers should be aware that scholars have largely explored consumers' acceptance by using different metrics such as: healthiness perception (Ares and Gambaro, 2007), willingness to try (Ares and Gambaro, 2007), willingness to buy (Siegrist et al., 2008), perceived fit (Krutulyte et al., 2011), attractiveness (Van Kleff et al., 2005a), intention to try (Van Kleff et al., 2005a) and willingness to purchase (Ares et al., 2010a). Due to the subjective valuation of hypothetical product concepts, and to the multitude of metrics used, results vary largely across studies (Van Kleff et al., 2005b).

${ }^{8}$ A carrier can be defined as the general product (such as yogurt, cereals, bread and juice), which serves as a vehicle for hosting a functional ingredient and/or a health claim.

${ }^{9}$ Consumers often perceive functional foods as "unnatural" and unhealthy since they are processed foods, while they more often consider healthy those described as "natural," "home-made," and "unprocessed" (Niva, 2006; Landström et al., 2009).
} 
Trestini (2014), using north-eastern Italian households' purchase data of fruit beverages found that health claims result in a $20.6 \%$ premium, while nutritional claims result in a $5.7 \%$ premium.

Third, the extant literature reports that consumers' choice of functional yogurts over conventional ones is significantly affected by a product's brand. Consumers show higher level of confidence in a functional product if this is sold with a brand they are familiar with, and tend to show higher level of acceptance for it, compared to consumers who are not familiar with the brand (Ares et al., 2010a, 2010b; Annunziata and Vecchio, 2013). ${ }^{10}$ Brand was also found as the most relevant attribute affecting consumers' functional yogurt choices among Italian (Annunziata and Vecchio, 2013) and Uruguayan consumers (Ares et al., 2010b). However, only limited empirical evidence using market level data exists to validate the importance of brands for the acceptance of functional products: Bonanno, Huang and Liu (2015) find that, among Italian consumers of yogurt, the utility associated with a given claim varies largely across brand. As a result, brand familiarity could affect the premium price associated to health claim.

\section{Data, methodology and model specification.}

We follow the standard hedonic price model proposed by Rosen (1974). According to this framework, each consumer chooses an optimal bundle of attributes which maximizes her/his utility (subject to a budget constraint), while manufacturers maximize profits by setting the price of the products they sell, given the attributes contained in the products produced. If each product in the market represents a unique bundle of attributes, at the equilibrium, marginal bids of buyers and marginal offer of sellers will match, whose envelope will result in a hedonic price function (Ladd and Suvannunt, 1976; Rosen, 1974).

The price of product $j$ in market $m$ at time $t, P_{j m t}$, can be described by the following function:

$$
P_{j m t}=f\left(X_{j m t}\right)(1)
$$

where $X$ is a vector of attributes and $f($.) is an unspecified functional form. Equation (1) is a reduced form equation implying that the price of product $j$ in market $m$ at time $t$ embeds the marginal monetary values of $j$ 's attributes (Ladd and Suvannunt,1976), which can be obtained by partially differentiating (1) with respect to each attribute.

In our case $X$ is partitioned in seven vectors: $X^{T C}, X^{H C}, X^{F B}, X^{O C}, X^{P}, X^{R}$ and $X^{B}$. The vectors $X^{T C}, X^{H C}$, and $X^{F B}$ include variables capturing the three factors affecting health claim's market value illustrated above: $X^{T C}$ ( $T C$ stands for "Type of Claim") represents a vector of variables indicating whether a product contains a specific functionality (namely cholesterol reduction, supporting the immune system, promoting bowel regularity, and bone health) and it is indexed by $t(t=1, \ldots, 4) ; X^{H C}$ ("Health Claim") is a vector of variables capturing whether a

\footnotetext{
${ }^{10}$ For an extensive discussion of theories about the role of brand in consumer food choices in presence of credence attributes please see Lassoued and Hobbs (2015).
} 
product carries a "reduction disease risk" $(R D)$ or a "general function" $(G F)$ claim so that $X^{H C}$ is indexed by $h=(1,2)$; last, $X^{F B}$ ("Familiar Band") includes two indicator variables capturing whether a given health claim belongs to a familiar brand or not, indexed by $f=(1,2)$. Our hypotheses are that health claims contribute positively to yogurt prices, and that: the better the match between the carrier (i.e. yogurt) and the specific claim, the higher the level of efficacy guaranteed on human health, and the more established the market presence of the brand; the higher the premium associate with a health claim will be.

The remaining four vectors capture other product characteristics which, based on previous literature (e.g. Carlucci et al., 2013) are expected to affect the price of yogurt in the Italian market. The vector $X^{O C}$ includes intrinsic product characteristics other than health claims (such as fat content, flavors, texture etc...), indexed by $o(o=1, \ldots, O)$; the vectors $X^{P}$ and $X^{R}$ include, respectively, packaging (type and size) and retail-level characteristics (depth and length of distribution), indexed by $p(p=1, \ldots, P)$ and $r(r=1, \ldots, R)$. Last, $X^{B}$ is a vector of brand fixed effects indexed by $b(b=1, \ldots, B)$. Details regarding model specification and functional form selection process are discussed later in the manuscript.

To capture the synergic interactions of the three factors affecting health claim's market value, that is, the vectors $X^{T C}, X^{H C}$, and $X^{F B}$, the variables included in these vectors were interacted with each other. The health claims were classified according to the type of benefit given to the consumers (supporting the immune system: Imm; cholesterol reduction: Chol; promoting bowel regularity: Reg; and supporting bone health: BoneH, elements of the vector $X^{T C}$ ), and on whether they represented "reduction disease risk" $(R D)$, or "general function" $(G F)$ claims (vector $X^{H C}$ ). For example, a product whose label reported the statement "reduces $L D L$ cholesterol by ... \% in ... weeks" (or similar) was classified as carrying a cholesterol lowering, reduction disease risk claim, as it is connected with the reduction of a risk factor in the development of coronary heart diseases. The indicator variable Chol_RD takes the value of 1 for those products carrying such claim, and 0 otherwise. Using the same logic, the indicator Chol_GF captures a product with the "weaker," general function cholesterol reduction claim, supported by statements such as "it contributes to the maintenance of normal blood cholesterol levels" or similar. One in ten products in our sample carries a health claim: the health claim most represented in our data is "supporting the immune system" (Imm_GF) carried by $37.3 \%$ of functional yogurts. Yogurts claiming to reduce (Chol_RD) or contributing to reduce (Chol_GF) blood cholesterol, account for $24.7 \%$ of the functional products in our data, while those promoting bowel regularity (Reg_GF) and supporting bone health (BoneH_GF) account for to $25.3 \%$ and $12.7 \%$ of our sample, respectively. All products with health claims in our sample carry only one claim.

The health claims were further divided in two groups to capture whether they were supported by brands with established market presence. We used national sales level as a proxy for how "familiar" a brand is, as suggested by Ares et al. (2010b). The vector $X^{F B}$ contains two indicator variables, one capturing products sold by a brand with a considerable market presence, i.e. a "familiar" brand, $(F B)$; another for those supported by one of the 200 brands whose joint presence accounts for less than $5 \%$ of sales in the Italian yogurt market, classified 
as "less-familiar" brand $(L F B)$. Thus, claims that are supported by a familiar brand, carry the suffix $F B$, while the others carry the suffix $L F B$.

The other vectors of variables are included in the model in additive fashion. Besides "traditional" product characteristics (fat content, flavors, texture), the vector $X^{O C}$ includes also variables capturing lactose free products (Lactose_Free), those added with fibres (Fibre), and whether a product was sold as "organic" (Organic) or "natural" (Natural). The last two variables are of importance since consumers seem to perceive products made with organic ingredients and those labelled "natural" as healthier and for which they are willing to pay a premium (Rozin et al., 2004; Onken et al., 2011).

The vector of packaging variables $X^{P}$ includes variables capturing whether the yogurt was sold in glass packaging (Glass) or in a two-compartment package (Compart_Pack). Package size was captured by three indicator variables, Regular, Medium, and Large, which recorded whether a yogurt was sold in packages with size smaller than $300 \mathrm{ml}$, between $300 \mathrm{ml}$ and $500 \mathrm{ml}$, and larger than $500 \mathrm{ml}$, respectively. The retailing variables capturing products availability in the retail channel $\left(X^{R}\right)$ are the number of product items of each manufacturer present on the stores' shelves (NumberItems), the percentage of stores selling the product (\%_Stores_Selling) and the average weighted distribution (AWD). Last, we included the vector $X^{B}$, containing brand-level indicator variables, or brand-specific fixed, in order effects to control further for unobserved brand-specific product characteristics. ${ }^{11}$

The main database used in the estimation comes from SymphonyIRI Group and contains monthly information on yogurt sales in 17 IRI Italian regions, ${ }^{12}$ covering the 25 -month period from November 29, 2010 to December 31, 2012. The data contains information on volume sold and value of sales, price (EUR/L) and products availability in the retail channel. The IRI data contain product unique item code (EAN) along with detailed information on manufacturers, brands, flavors, fat content, drinkability, whether a yogurt is sold as smoothie or if it has an additional compartment (with cereals, chocolate chips, etc..) and categorizes functional yogurts under the general umbrella term of "sante" (health), and provides information that allows us to identify the different claims carried by the products in the "sante" category.

To validate the types of health claim present in the data, we retrieved information from product images available on the manufacturers' websites, specifically from the products' front-of-package claims and nutritional labels. In our data, we identified 327 products 13 carrying unique combinations of attributes, encompassing 77 brands sold by 21 manufacturers for a total of 60,011observations. Summary statistics of the different product attributes, as well as a description of the variables used in the model, are presented in Table 1.

\footnotetext{
${ }^{11}$ As discussed later in the manuscript we performed a test to verify a fixed-effect or a random effect model fits the data best. Please see footnote 15 for more details.

${ }^{12}$ Although the Italian regions are 20, Symphony IRI groups data from Piedmont and Aosta Valley, Abruzzo and Molise, and Basilicata and Calabria, resulting in 17 "IRI regions".

${ }^{13}$ Private labels, whose attributes could not be verified, were excluded from the analysis along with products classified by SymphonyIRI Group as having "other functionality". We focus our analysis on yogurt market for adults made from cow milk, yogurt made from non-cow milk as well as yogurt for kids were excluded from our analysis.
} 
Chapter 3

Table 1. Descriptive statistic (60,011 observations).

\begin{tabular}{|c|c|c|}
\hline Variable & Definition & $\begin{array}{l}\text { Mean }^{\text {a }} \\
\text { (Std. Dev.) }\end{array}$ \\
\hline Price & yogurt price EUR/ liter & $\begin{array}{l}4.305 \\
(1.480)\end{array}$ \\
\hline \multicolumn{3}{|l|}{ Health Claims } \\
\hline Chol_RD_FB & $\begin{array}{l}1=\text { yogurt with cholesterol reduction disease claim, } \\
\text { supported by familiar brand }\end{array}$ & 0.016 \\
\hline Chol_GF_FB & $\begin{array}{l}\text { 1= yogurt with cholesterol lowering general function } \\
\text { claim, supported by familiar brand }\end{array}$ & 0.014 \\
\hline Chol_GF_LFB & $\begin{array}{l}\text { 1= yogurt with cholesterol lowering general function } \\
\text { claim, supported by less familiar brand }\end{array}$ & 0.009 \\
\hline$I m m_{-} G F_{-} F B$ & $\begin{array}{l}1=\text { yogurt with immune system support claim, supported } \\
\text { by familiar brand }\end{array}$ & 0.050 \\
\hline$I m m \_G F \_L F B$ & $\begin{array}{l}1=\text { yogurt with supporting the immune claim, supported by } \\
\text { less familiar brand }\end{array}$ & 0.009 \\
\hline Reg_GF_FB & $\begin{array}{l}\text { 1= yogurt with promoting bowel regularity claim, } \\
\text { supported by familiar brand }\end{array}$ & 0.028 \\
\hline$R e g \_G F \_L F B$ & $\begin{array}{l}\text { 1=yogurt with promoting bowel regularity claim, } \\
\text { supported by less familiar brand }\end{array}$ & 0.012 \\
\hline BoneH_GF_FB & $\begin{array}{l}\text { 1= yogurt with supporting bone health claim, supported by } \\
\text { familiar brand }\end{array}$ & 0.020 \\
\hline \multicolumn{3}{|c|}{ Other Product Characteristics } \\
\hline Plain & $1=$ plain yogurt & 0.301 \\
\hline Fruit & $1=$ fruit flavored yogurt & 0.488 \\
\hline Others_flavour & $1=$ yogurt with flavour mix & 0.209 \\
\hline RegularFat & $1=$ yogurt with regular fat content & 0.301 \\
\hline Low_Fat & $1=$ yogurt with reduced fat content & 0.259 \\
\hline Zero_Fat & $1=$ yogurt with $0 \%$ fat content & 0.248 \\
\hline Lactose_Free & $1=$ lactose free yogurt & 0.017 \\
\hline Drinkable & $1=$ drinkable yogurt & 0.231 \\
\hline Fibre & $1=$ yogurt added with fibre & 0.065 \\
\hline Natural & $1=$ yogurt labeled as natural & 0.013 \\
\hline Organic & $1=$ yogurt made from organic milk & 0.068 \\
\hline Smoothie & $1=$ smoothie & 0.002 \\
\hline \multicolumn{3}{|l|}{ Packaging } \\
\hline Glass & $1=$ yogurt sold in glass packages & 0.019 \\
\hline Compart_Pack & $1=$ yogurt sold in two compartment packages & 0.097 \\
\hline Regular & $1=$ package size $<300 \mathrm{ml}$ & 0.750 \\
\hline Medium & $1=$ package size between $300 \mathrm{ml}$ and $500 \mathrm{ml}$ & 0.249 \\
\hline \multicolumn{2}{|l|}{ Retail variables } & 0.001 \\
\hline Number of Items & $\begin{array}{l}\text { Number of product items of each manufacturers on store's } \\
\text { selves }\end{array}$ & $\begin{array}{l}3.182 \\
(3.513)\end{array}$ \\
\hline$\%$ Stores_Selling & Percentage of stores selling the product & $\begin{array}{l}0.147 \\
(1.398)\end{array}$ \\
\hline$A W D$ & $\begin{array}{l}\text { Percentage of outlets selling the product conditional on the } \\
\text { manufacturer's products being available in a given store }\end{array}$ & $\begin{array}{l}30.184 \\
(28.216)\end{array}$ \\
\hline
\end{tabular}

\footnotetext{
Source: SymphonyIRI Group InfoScan yougurt database.

${ }^{a}$ For all binary variables the standard deviation is omitted as the mean, reported in table, represents the share of observations showing value of 1 .
} 
Following previous literature (e.g. Costanigro et al., 2007; Carlucci et al., 2013; Szathvary and Trestini, 2014; Bonanno, forthcoming), we estimate the parameters of equation (1) using a single equation approach. We select the appropriate functional form for $f($.$) from ten$ different transformations of the dependent variable. Following Constanigro et al. (2007) we perform a grid search involving 8 discrete choices of the dependent variable, in the form $P^{\alpha}$, where $\alpha$ varies from -2 to +2 with increments of 0.5 ( $\alpha=0$ excluded), and the natural $\log$ transformation. We select the most suitable model specification using the following criteria, as in Constanigro et al.(2007): Ramsey's RESET test (Ramsey, 1969) for omitted variable bias; Breusch-Pagan / Cook-Weisberg statistic for heteroskedasticity (Breusch and Pagan, 1979; Cook and Weisberg, 1983); Skewness and Kurtosis test for the normality of the error terms (D’Agostino et al., 1990); and goodness of fit (Adjusted $R^{2}$ ). Table 2 provides a summary of the test statistics' values for the 10 model specifications resulting from transformations of the dependent variable.

Table 2. Model Diagnostics used for model selection.

\begin{tabular}{|c|c|c|c|c|c|}
\hline \multirow{4}{*}{$\begin{array}{l} \\
\text { Transf. } \\
-2\end{array}$} & \multicolumn{2}{|c|}{ Model Fit } & \multirow{2}{*}{$\begin{array}{l}\text { Specification } \\
\text { Ramsey's } \\
\text { RESET }\end{array}$} & \multirow{2}{*}{$\begin{array}{l}\text { Heteroskedasticity } \\
\text { Breusch-Pagan / } \\
\text { Cook-Weisberg }\end{array}$} & \multirow{2}{*}{$\begin{array}{l}\text { Normality } \\
\text { Skewness and } \\
\text { Kurtosis }\end{array}$} \\
\hline & Adj $R^{2}$ & $F$-stat & & & \\
\hline & & & $\mathrm{F}(3,59883)$ & $\operatorname{chi}^{2}(1)$ & $\operatorname{chi}^{2}(2)$ \\
\hline & 0.7254 & 1279.70 & 1824.44 & 78477.70 & 48759.70 \\
\hline-1.5 & 0.7490 & 1444.86 & $\begin{array}{l}(0.0000) \\
963.71 \\
(0.0000)\end{array}$ & $\begin{array}{l}(0.0000) \\
29592.58 \\
(0.0000)\end{array}$ & $\begin{array}{l}(0.0000) \\
30673.02 \\
(0.0000)\end{array}$ \\
\hline-1 & 0.7629 & 1557.96 & $\begin{array}{l}408.74 \\
(0.0000)\end{array}$ & $\begin{array}{l}9917.71 \\
(0.0000)\end{array}$ & $\begin{array}{l}18175.36 \\
(0.0000)\end{array}$ \\
\hline-0.5 & 0.7693 & 1615.20 & $\begin{array}{l}101.85 \\
(0.0000)\end{array}$ & $\begin{array}{l}2131.61 \\
(0.0000)\end{array}$ & $\begin{array}{l}10069.09 \\
(0.0000)\end{array}$ \\
\hline $\begin{array}{l}\text { Box-Cox } \\
(0.138)\end{array}$ & 0.7684 & 1606.89 & $\begin{array}{l}10.95 \\
(0.0000)\end{array}$ & $\begin{array}{l}119.71 \\
(0.0000)\end{array}$ & $\begin{array}{l}4530.75 \\
(0.0000)\end{array}$ \\
\hline Log & 0.7694 & 1616.00 & $\begin{array}{l}1.52 \\
(0.2072)\end{array}$ & $\begin{array}{l}0.53 \\
(0.4668)\end{array}$ & $\begin{array}{l}5190.24 \\
(0.0000)\end{array}$ \\
\hline 0.5 & 0.7634 & 1562.22 & $\begin{array}{l}123.57 \\
(0.0000)\end{array}$ & $\begin{array}{l}1892.97 \\
(0.0000)\end{array}$ & $\begin{array}{l}4832.17 \\
(0.0000)\end{array}$ \\
\hline 1 & 0.7629 & 1459.27 & $\begin{array}{l}494.19 \\
(0.0000)\end{array}$ & $\begin{array}{l}8141.35 \\
(0.0000)\end{array}$ & $\begin{array}{l}12113.60 \\
(0.0000)\end{array}$ \\
\hline 1.5 & 0.7310 & 1316.45 & $\begin{array}{l}1107.08 \\
(0.0000)\end{array}$ & $\begin{array}{l}19902.38 \\
(0.0000)\end{array}$ & $\begin{array}{l}26893.00 \\
(0.0000)\end{array}$ \\
\hline 2 & 0.7029 & 1145.83 & $\begin{array}{l}1846.18 \\
(0.0000)\end{array}$ & $\begin{array}{l}38613.02 \\
(0.0000)\end{array}$ & $\begin{array}{l}45968.89 \\
(0.0000)\end{array}$ \\
\hline
\end{tabular}

Note: Probability values in parenthesis below the test statistics.

Based on Ramsey's RESET test results, the semi-logarithmic specification emerges as the most suitable. For the same specification, the Breusch-Pagan/Cook-Weisberg statistic indicates constant variance of the residuals. 
Also, the semi-logarithmic specification performs best in terms of fit, showing the largest adjusted $R^{2}(0.7694)$ and the largest value of the $F$ statistic for the coefficients' joint significance. Although Skewness and Kurtosis' tests formally reject the hypothesis of the errors being normally distributed, the residuals of the semi-logarithmic specification were the closest to be normally distributed among the different model specifications. ${ }^{14}$

Thus, given the vectors of variables in $X$ and the semi-logarithmic functional form, the chosen empirical specification of equation (1) is:

$$
\begin{aligned}
\ln P_{j m t}=\sum_{h=1}^{H} \sum_{t=1}^{T} \sum_{f=1}^{F} \alpha_{h t f} X_{h m t}^{H C} X_{t m t}^{T C} X_{f m t}^{F B}+\sum_{o=1}^{O} \beta_{o} X_{o m t}^{O C}+\sum_{p=1}^{P} \beta_{p} X_{p m t}^{P}+\sum_{r=1}^{R} \beta_{r} X_{r m t}^{R} \\
+\sum_{b=1}^{77} \gamma_{b} X_{b m t}^{B}+\sum_{m=1}^{17} \lambda_{m} d_{m}+\sum_{t=1}^{25} \theta_{t} d_{t}+\epsilon_{j m t}(2)
\end{aligned}
$$

where we also control for a vector of $M$ market-level (region) and $T$ time (month) indicators, $d_{m}$ and $d_{t}$, respectively to capture regional and monthly variation in yogurt prices. The $\alpha_{\mathrm{s}}, \beta_{s}$, are parameters to be estimated capturing, respectively, the implicit prices associated with health-claims and their features, those of other product characteristics, packaging and retailing characteristics. The terms $\gamma_{b}, \lambda_{m}$ and $\theta_{t}$ capture, respectively, brand, regional and time (monthly) fixed-effects, while $\epsilon_{\text {jmt }}$ is an idiosyncratic error term. ${ }^{15}$

\section{Empirical Results and Discussion}

The estimated parameters of equation (2) are presented in the first column of Table 3, along with their standard errors (clustered by brand and month) in the second column. The marginal prices of each attribute (in percentage terms), calculated using Kennedy's (1981) adjustment for indicator variables, and in elasticity terms for the continuous ones (at the sample averages) are reported in the last column. The baseline product is a regular fat, plain, non-drinkable yogurt, sold in plastic packaging $(<300 \mathrm{ml})$ with an average price of $4.30 \mathrm{EUR} / \mathrm{L}$.

The first finding we can highlight is that, in the Italian yogurt market all health claims indicators have a positive and significant effect on prices. Among the health claims tested, cholesterol risk reduction claim Chol_RD_FB, shows the highest implicit price, $+222.6 \%$ the price of the baseline alternative, followed by the respective general function claim

\footnotetext{
${ }^{14}$ We also estimated the model using Box Cox transformations, using power transformations of the explanatory variable in the form $y^{\lambda}$ where $\lambda$ takes the values $-1,0$, and 1 . The value of the maximized likelihood function for three estimated models was the highest when $\lambda=0$, suggesting a log-linear transformation of the dependent variable to be preferred over linear $(\lambda=1)$ and inverse $(\lambda=-1)$, similar to Loureiro and McCluskey (2000), Huang and Lin (2007) and Szathvary and Trestini (2013)

${ }^{15}$ We estimated a random-effect model first and then, we followed Arellano (1993) and Wooldridge's (2002) approach to select which estimator (fixed-effects or random-effects) provided consistent and efficient estimates. We obtained a test statistic of 114.335 distributed $\chi_{(43)}^{2}$, above the critical value (at the $1 \%$ level) of 77.419 , suggesting that the fixed-effect estimator is to be preferred to the random effect one, also suggesting that unobserved heterogeneity may not be a large source of bias for our estimates. We thank two anonymous reviewers for suggesting we performed such test.
} 
Chapter 3

$\left(C h o l \_G F \_F B\right)+85.5 \%$, both claims supported by familiar brands. These estimates are consistent with other literature indicating products claiming to prevent or reduce the risk of cardiovascular diseases by lowering blood cholesterol level to be well accepted by dairy product consumers (Ares and Gambaro, 2007; Landström et al., 2007).

Table 3. Estimated parameters and percentage premium price.

\begin{tabular}{|c|c|c|c|c|}
\hline Variable & $\beta$ & S.E. & Significance & $\begin{array}{l}\text { Percentage } \\
\text { premium price }\end{array}$ \\
\hline \multicolumn{5}{|c|}{ Health Claims variables } \\
\hline Chol_RD_FB & 1.172 & 0.039 & $* * *$ & +222.6 \\
\hline Chol_GF_FB & 0.618 & 0.025 & $* * *$ & +85.5 \\
\hline Chol_GF_LFB & 0.125 & 0.023 & $* * *$ & +13.3 \\
\hline$I m m_{-} G F_{-} F B$ & 0.127 & 0.026 & $* * *$ & +13.5 \\
\hline$I m m_{-} G F \_L F B$ & 0.417 & 0.019 & $* * *$ & +51.7 \\
\hline$R e g \_G F \_F B$ & 0.178 & 0.031 & $* * *$ & +19.4 \\
\hline Reg_GF_LFB & 0.060 & 0.014 & $* * *$ & +6.2 \\
\hline BoneH_GF_FB & 0.412 & 0.040 & $* * *$ & +50.9 \\
\hline \multicolumn{5}{|c|}{ Other Product Characteristics variables } \\
\hline Fruit & 0.032 & 0.004 & $* * *$ & +3.2 \\
\hline Others_flavour & 0.065 & 0.006 & $* * *$ & +6.7 \\
\hline Low_Fat & -0.044 & 0.013 & $* * *$ & -4.3 \\
\hline Zero_Fat & -0.019 & 0.008 & $* * *$ & -1.9 \\
\hline Lactose_Free & 0.072 & 0.020 & $* * *$ & +7.4 \\
\hline Drinkable & 0.177 & 0.016 & $* * *$ & +19.3 \\
\hline Fibre & -0.052 & 0.011 & $* * *$ & -5.0 \\
\hline Natural & 0.704 & 0.034 & $* * *$ & +102.0 \\
\hline Organic & 0.383 & 0.022 & $* * *$ & +46.6 \\
\hline Smoothie & 0.044 & 0.022 & $*$ & +4.5 \\
\hline \multicolumn{5}{|c|}{ Packaging variables } \\
\hline Glass & 0.860 & 0.034 & $* * *$ & +136.1 \\
\hline Compart_Pack & 0.434 & 0.022 & $* * *$ & +54.3 \\
\hline Medium & -0.103 & 0.01 & $* * *$ & -9.8 \\
\hline Large & -0.249 & 0.032 & $* * *$ & -22.1 \\
\hline \multicolumn{5}{|l|}{ Retailing variables } \\
\hline Number of Items & -0.006 & 0.000 & $* * *$ & $-0.0190 \S$ \\
\hline$\%$ \%tores_Selling & -0.002 & 0.000 & $* * *$ & $-0.0003 \S$ \\
\hline$A \bar{W} D$ & -0.001 & 0.000 & $* * *$ & $-0.0302 \S$ \\
\hline Constant & 0.7709 & 0.034 & $* * *$ & \\
\hline
\end{tabular}

Note: Standard errors adjusted for clustering on brand interacted by month. The estimated coefficients for regional fixed effects, brand and monthly indicators are omitted for brevity.

$\neq$ Adjustment made according to Kennedy (1981).

$\S$ For continuous variables we present average elasticity values.

$*$,** and *** are 10. 5 and $1 \%$ significance levels. 
The other functional claims carried by familiar brands show positive premiums also: bone health (BoneH_GF_FB), promoting bowel regularity $\left(R e g \_G F \_F B\right)$, and strengthening the immune system claims (Imm_GF_FB), have effects on yogurt prices of $+50.9 \%,+19.4 \%$ and $+13.5 \%$, respectively (compared to the baseline option). The fact that the second highest marginal price for health claims is that of bone health, may be due to the high consumer acceptance of dairy product added with calcium as well as the general knowledge of the functions that calcium plays in organism due to the effect of public health campaigns (Ares and Gámbaro, 2007). "Promoting bowel regularity" claims (Reg_GF_FB ) show one of lowest premium price of $+19.4 \%$, comparable with that obtained by Carlucci et al.(2013) of $+24.45 \%$. Such low premium price may be due to the market of these products approaching maturity (Siró et al., 2008), since yogurt with live microorganisms was one of the first functional food launched in the European market over 15 years ago (Menrad, 2003).

Claims strengthening the immune system supported by familiar brands $\left(I m m_{-} G F_{-} F B\right)$ lead to the lowest premium, 4 times lower than that associated with less familiars brands, which is $+51.7 \%$. This result may be a consequence of the reformulations of products carrying these claims, once being supported by microorganisms, and more recently by vitamins (Nutraingredients, 2012) ${ }^{16}$. Therefore consumers may have lost trust towards immune claims supported by familiar brand which, ultimately, leads to a lower price premium. An alternative explanation of this result is that, as consumers may become familiar with functional ingredients, like vitamins and microorganisms, as well as with the immune related benefits that they provide (Wills et al., 2012), immunity claims can develop a brand-like effect, acting as a quality signal, similar to what Hassan and Monier-Dilhan (2006) found in the context of Protected Denomination of Origin (PDO) products. ${ }^{17}$

Another finding worth pointing out is that the only risk reduction claim in our data Chol_RD_FB, has an implicit price 2.5 times larger than the respective general function claim Chol_GF_FB. These estimates confirm that the presence on the label of heart diseases risk reduction claim increases consumers' acceptance of functional dairy more than what general function claims do (Van Kleef et al., 2005a; Verbeke et al., 2009).

Our results confirm only in part the hypothesis that claims supported by familiar brands outperform those carried by lesser familiar ones. In two out of three cases the premium attached to claims supported by familiar brands exceeds, from 6.4 to 3 times, that of lesser familiar brands, confirming brand as an important determinant for functional products success (Messina et al., 2008; Ares et al., 2010a, 2010b; Annunziata and Vecchio, 2013). The only exception was for Imm_GF_LFB.

\footnotetext{
${ }^{16}$ Prior to the implementation of Regulation (EC) No.1924/2006, manufacturers employed mixtures of bacteria to support their immunity health claim; as EFSA no longer admits the addition of microbes to support that claim (Reg. EC. 432/2012) manufacturers have started using vitamins instead.

${ }^{17}$ Hassan and Monier-Dilhan (2006) found that the PDO attribute resulted in a positive large effect on the implicit price of products sold as a less familiar brand (private label), and in a price discount for well-known brands.
} 
The other credence attributes, Organic and Natural, show positive and significant effects on yogurt price in Italy of, respectively, $+46.6 \%$ and $+102.0 \%$ over the baseline product's price. The high premiums of these attributes are likely to be the result of consumers' high willingness to pay for characteristics having a "halo effect" (Schuldt, 2013). In fact, products labeled as "Organic" and "Natural," are often perceived healthier than regular ones ${ }^{18}$ and consumers' primary reason for buying organic foods is their belief that these products may support human health (Hughner et al., 2007). Additionally, the premium associated to Organic is in line with estimates by Carlucci et al. (2013), and Bimbo et al. (forthcoming) in the Italian yogurt and UHT milk market, respectively. Organic attribute, in fact, showed a premium price of $+28.8 \%$ on yogurt (Carlucci et al.,2013) and a higher premium $(+73.5 \%)$ for UHT milk for general population (Bimbo et al., forthcoming). Although no other study conducted in the Italian market exists estimating a premium price for the Natural attribute, evidence from the U.S. yogurt market (Bonanno, fortcoming) shows a pattern similar to that found here, with an estimated Organic premium of circa one-third of Natural's $(12.6 \%$ vs $35.1 \%)$.

The estimated implicit prices of the other product characteristics are relatively small: fruits and other flavors show a positive effect of $+3.2 \%$ and $+6.7 \%$, respectively, compared to the baseline alternative, while lower fat contents affect yogurt prices negatively. The marginal prices of the Low_Fat and Zero_Fat attributes are $-4.3 \%$ and $-1.9 \%$, respectively, partially in line with Carlucci et al.(2013) who found a negative but not significant relationship between the "low-fat" attribute and yogurt's price. The Drinkable attribute shows a positive and significant effect on price $(+19.3 \%$ compared to the baseline product), which seem consistent with Bonanno's (2013) finding that Italian yogurt consumers seem to prefer drinkable yogurts over regular ones, in particular with regard to functional alternatives. The Smoothie and Lactose_Free attributes show a positive premium of $+4.5 \%$ and $+7.4 \%$, respectively, while the presence of added fibre (Fibre) leads to a $-5.0 \%$ markdown. Although our finding of a discount for the presence of fibres in yogurt contrasts with Carlucci et al.'s (2013), Bonanno (forthcoming) finds a markdown associated with this attribute in the U.S. yogurt market of $7.6 \%$. The presence of a markdown can be justified by findings of other studies highlighting consumers' skepticism for attributes which are "unnatural" or artificially added to a product (Krutulyte et al., 2008). Fibres added to yogurt are perceived as interfering with the naturalness and healthiness of the product and this may reduce consumers' acceptance and willingness to try (Ares and Gambaro, 2007), and therefore its price.

The marginal prices associated with the packaging variables are in line with previous findings (Carlucci et al., 2013; Szathvary and Trestini, 2014) showing a higher price for yogurts sold in glass packaging (Glass) and those with an additional compartment (Compart_Pack) for premiums of $+136.1 \%$ and $+54.3 \%$, respectively. The first result may be due to glass packaging being used by consumers to infer yogurt wholesomeness (Grunert 2005b), and may also reflect the higher cost of the packaging material, as suggested by Silayoi and Speece

\footnotetext{
${ }^{18}$ Terms such as "sustainable," "organic," "genetically-modified free", "unprocessed”, "natural," and "home-made," are usually used by consumers to describe healthy products (Rozin, 1994; Schuldt, 2013).
} 
(2004). The estimated negative effects of products sold in larger package sizes (by $-9.8 \%$ and $-22.1 \%$, respectively, for package size between $300 \mathrm{ml}-500 \mathrm{ml}$, and more than $500 \mathrm{ml}$ ) indicate that yogurt price declines with size, as expected. Lastly, yogurt's price seems to decrease as products' availability in the retail channel increases, as suggested by the estimated average elasticities for Number of Items (-0.0190), \%Stores_Selling (-0.0003), and AWD (-0.0302).

\section{Conclusions}

In spite of their market growth, functional foods show high failure rates, which may be due to the manufacturers inability to capitalize on the different factors determining a functional product's success. Among the large body of literature assessing consumers' attitudes towards functional products, one can identify three aspects which influence the acceptance of health claims, and therefore the premium associated with them: type of functionality, the level of efficacy guaranteed by the claim, and the support of a familiar brand. In this paper, we use Italian yogurt sales data and a hedonic price model to estimate the contribution of these three factors on yogurt price. Our estimates show different premiums associated with different claimed benefits. The claims with the highest marginal prices are those lowering / managing the cholesterol blood level, and those supporting bone health. Thus, yogurt seems a wellsuited carrier to host calcium or phytosterols and using these ingredients may constitute valid differentiation tools for manufacturers with limited resources to invest in "risk disease reduction" claims.

Our findings also suggest that a claim's efficacy matters in determining a product's premium price: in the case of cholesterol lowering products, reduction disease risk claims have a much larger marginal price compared to general function ones. Thus, our results suggest that the high investments required to obtain approval for a reduction of disease claim may actually pay off, as manufacturers may benefit from considerably higher prices. Furthermore, we find that most health claims supported by a familiar brand, benefit from an additional premium price compared to those supported by less familiar ones. Therefore, firms investing in the development of functional products may need to invest first in building and sustaining their brand image as a mean to increase health claim' marginal price. Alternatively, firms may prefer to invest in health claims associated with well-known functional ingredients/benefit, in order to obtain a claim with brand-like effect. Last, our results indicate that manufacturers may benefit from differentiating their products not only on the basis of health claims but also using other credence attributes such as "organic" or "natural," as the estimated implicit prices of those attributes is comparable to those of some of the health claims in our data.

In spite of our results' usefulness to provide guidance to food manufacturers in deciding whether or not to invest in the development and marketing of functional products, our analysis shows some limitations. First, our results do not provide insights on the role played by consumers' heterogeneity in the process of price formation, as they are obtained using aggregate market level data. Second, although we considered the level of efficacy as a factor influencing the premium attached to a health claim, we fail to consider other claim-specific 
factors, such as its complexity, or whether framing was positive or negative. As these aspects affect consumers' acceptance of functional products (Grunert et al., 2009) our failure to account for them may bias our estimated implicit price. Third, the data at our disposal did not allow us to account for timing and types of products reformulations which may have occurred during the period in analysis the results presented may, at least potentially, show some bias, or represent (weighted) average premiums for claims before and after reformulation occurred, which may limit the relevance of the magnitude discussed. Fourth, and last, our estimated effects represent the average contribution of yogurt's features on price and it does not allow for non-linearities or other effects of the attributes on prices. Therefore future research could use more detailed household-level purchase data and more powerful and flexible models and estimation techniques (e.g. quantile regression) to tackle these limitations.

\section{Acknowledgement}

The research leading to these results has received funding from the Apulian Regional Project. LAIFF - Laboratories Network for the Innovation on Functional Foods. Grant n.47. PO Puglia FESR 2007-2013, Asse I, Linea 1.2.

\section{References}

Annunziata, A. and Vecchio, R. (2013). Consumer perception of functional foods: a conjoint analysis with probiotics. Food Quality and Preference 28: 348-355.

Arellano, M. (1993). On the testing of correlated effects with panel data. Journal of Econometrics 59(1): 87-97.Ares, G. and Gámbaro, A. (2007). Influence of gender, age and motives underlying food choice on perceived healthiness and willingness to try functional foods. Appetite 49: 148-158.

Ares, G., Besio, M., Giménez, A. and Deliza, R. (2010a). Relationship between involvement and functional milk desserts intention to purchase' Influence on attitude towards packaging characteristics. Appetite 55(2): 298-304.

Ares, G., Giménez, A. and Deliza, R. (2010b). Influence of three non-sensory factors on consumer choice of functional yogurts over regular ones. Food Quality and Preference 21: 361-367.

Balcombe, K., Bitzios, M., Fraser, I. and Haddock-Fraser, J. (2014). Using attribute importance rankings within discrete choice experiments: an application to valuing bread attributes. Journal of Agricultural Economics 65(2): 446-462.

Barreiro-Hurlé, J., Colombo, S. and Cantos-Villar, E. (2008). Is there a market for functional wines? Consumer preferences and willingness to pay for resveratrol-enriched red wine. Food Quality and Preference 19(4): 360-371.

Bimbo, F, Bonanno, A., Liu, X., Viscecchia, R. (forthcoming). Hedonic analysis of ultra-heat treated milk prices in Italy Journal of Dairy Science. 
Bonanno, A. (2012). Some like it healthy: Demand for functional and conventional yogurts in the Italian market. Agribusiness: an International Journal. 28: 67-85.

Bonanno, A. (2013). Functional foods as differentiated products: the Italian yogurt market. European Review of Agricultural Economics 40: 45-71.

Bonanno, A. (forthcoming). A Hedonic Valuation of Health and Non-Health Attributes in the U.S. Yogurt Market. Agribusiness: an International Journal.

Bonanno, A., Huang, R. and Liu, Y. (2015). Simulating welfare effects of the European nutrition and health claims' regulation: the Italian yogurt market. European Review of Agricultural Economics 42(3): 499-533.

Breusch, T. and Pagan, A. (1979). A simple test of heteroskedasticity and Rrandom coefficient variation. Econometrica 47: 1287-1294.

Brookes, G. (2010). Economic Impact Assessment of the European Union (EU)'s Nutrition \& Health Claims Regulation on the EU food supplement sector and market (European Health Claims Alliance EHCA).

Carlucci, D., Stasi, A., Nardone, G. and Seccia, A. (2013). Explaining price variability in the Italian yogurt market: a hedonic analysis. Agribusiness 29: 194-206.

Cook, R. D. and Weisberg, S. (1983). Diagnostics for heteroscedasticity in regression. Biometrika, 70: 1-10.

Costanigro, M., McCluskey, J. J. and Mittelhammer, R. C. (2007). Segmenting the wine market based on price: Hedonic regression when different prices mean different products. Journal of Agricultural Economics 58: 454-466.

D'Agostino, R.B., Balanger, A. and D'Agostino, R. B. Jr. (1990). A suggestion for using powerful and informative tests of normality. American Statistician 44: 316-321.

Ding, Y., Veeman, M. M. and Adamowicz, W. L. (2012). The influence of attribute cutoffs on consumers' choices of a functional food. European Review of Agricultural Economics 39(5): 745-769.

Diplock, A.T., Aggett, P.J., Ashwell, M., Bornet, F., Fern, E.B. and Roberfroid, M.B. (1999). Scientific concepts of functional foods in Europe: consensus document. British Journal of Nutrition 81: 1-27.Grunert, K.G. (2005a). Food quality and safety: consumer perception and demand. European Review of Agricultural Economics 33: 369-391.

Grunert, K.G. (2005). Innovation in Agri-food Systems: Product Quality and Consumer Acceptance. Wageningen Academic Publishers, The Netherlands.

Grunert, K. G., Lähteenmäki, L., Boztug, Y., Martinsdóttir, E., Ueland, Ø., Åström, A., \& Lampila, P. (2009). Perception of health claims among Nordic consumers. Journal of Consumer Policy, 32(3), 269-287.

Hailu, G., Boecker, A., Henson, S. and Cranfield, J. (2009). Consumer valuation of functional foods and nutraceuticals in Canada. A conjoint study using probiotics. Appetite 52: 257-265. 
Hassan, D., and Monier-Dilhan, S. (2006). National brands and store brands: competition through public quality labels. Agribusiness. 22: 21-30.

Hobbs, J. E., Malla, S. and Sogah, E. K. (2014). Regulatory Frameworks for Functional Food and Supplements. Canadian Journal of Agricultural Economics/Revue canadienne d'agroeconomie 62(4): 569-594.

Huang, C. L. and Lin, B-H. (2007). A hedonic analysis of fresh tomato price among regional markets. Review of Agricultural Economics 29: 783-800.

Hughner, R.S., McDonagh, P., Prothero, A., Shultz, C.J. II, and Stanton, J. (2007). Who are organic food consumers': A compilation and review of why people purchase organic food. Journal of Consumer Behaviour 6: 94-110.

Kennedy, P. E. (1981). Estimation with correctly interpreted dummy variables in semilogarithmic equations. The American Economic Review 71: 801.

Krutulyte, R., Scholderer, J., Hagemann, K., Grunert, G.K., Elgaard, P. and Nielsen B. (2008). Motivational factors for consuming omega-3 PUFAs: An exploratory study with Danish consumers. Appetite 51: 137-147.

Krutulyte R., Grunert, K. G., Scholderer, J., Lahteenmaki, L., Hagemann, K. S., Elgaard, P., Nielsen, B. and Graverholt J. P. (2011). Perceived fit of different combinations of carriers and functional ingredients and its effect on purchase intention. Food Quality and Preference 22(1): 11-16.

Ladd, G. W. and Suvannunt, V. (1976). A model of consumer goods characteristics. American Journal of Agricultural Economics 58: 504-510.

Landström, E., Hursti, U.K.K., Becker, W. and Magnusson, M. (2007). Use of functional foods among Swedish consumers is related to health-consciousness and perceived effect. British Journal of Nutrition 98: 1058-1069.

Landström, E., Hursti, U.-K.K. and Magnusson, M. (2009). Functional foods compensate for an unhealthy lifestyle. Some Swedish consumers' impressions and perceived need of functional foods. Appetite 53: 34-43.

Lassoued, R. and Hobbs, J. (2015). Consumer confidence in credence attributes: The role of brand trust. Food Policy 52: 90-107.

Loureiro, M. L. and McCluskey, J. J. (2000). Assessing consumer response to protected geographical identification labeling. Agribusiness 16: 309-320.

Malla, S., Hobbs, J. E. and Perger, O. (2007). Valuing the health benefits of a novel functional food. Canadian Journal of Agricultural Economics/Revue canadienne d'agroeconomie 55(1): 115-136.

Menrad, K. (2003). Market and marketing of functional food in Europe. Journal of food engineering 56(2): 181-188. 
Messina, F., Saba, A., Turrini, A., Raats, M., Lumbers, M. and Team, F. (2008). Older people's perceptions towards conventional and functional yoghurts through the repertory grid method: a cross-country study. British Food Journal 110: 790-804.

Moro, D., Veneziani, M., Sckokai, P. and Castellari, E. (2015). Consumer willingness to pay

for catechin enriched yogurt: evidence from a stated choice experiment. Agribusiness: an International Journal 31(2): 243- 258.

Niva, M. (2006). Can we predict who adopts health-promoting foods? Users of functional foods in Finland. Scandinavian Journal of Food and Nutrition 50: 13-24.

Nutraingredients, Digestive health products are No.1 functional food, global study (2009) available at http://www.nutraingredients-usa.com/Markets/Digestive-health-products-areNo.1-functional-food-global-study last access 6/10/2014.

Nutraingredients, EU immunity health claims: reformulate or reposition to win growth (2012) available at http://www.nutraingredients.com/Regulation/EU-immunity-health-claimsReformulate-or-reposition-to-win-growth last access 13/10/2014.

Onken, K.A., Bernard, J.C. and Pesek, J.D.Jr. (2011). Comparing willingness to pay for organic, natural, locally grown, and state marketing program promoted foods in the midAtlantic region. Agricultural and Resource Economics Review 40(1): 33-47.

Ozer, B.H. and Kirmaci, H.A. (2010). Functional milks and dairy beverages. International Journal of Dairy Technology 63: 1-15.

Ramsey, J.B. (1969). Tests for specification errors in classical linear least squares regression analysis. Journal of the Royal Statistical Society 31: 50-371.

Regulation (EC) No 1924/2006 of the European Parliament and of the Council of 20 December 2006 on nutrition and health claims made on foods.

Regulation (EC) No 432/2012. Establishing a list of permitted health claims made on foods, other than those referring to the reduction of disease risk and to children's development and health.

Rosen, S. (1974). Hedonic prices and implicit markets: product differentiation in pure competition. Journal of Political Economy 82: 34-55.

Rozin, P., Spranca, M., Krieger, Z., Neuhaus, R., Surillo, D., Swerdlin, A. and Wood, K. (2004). Natural preference: instrumental and ideational/moral motivations, and the contrast between foods and medicines. Appetite 43: 147-154.

Schuldt, J.P. (2013). Does green mean healthy? Nutrition label color affects perceptions of healthfulness. Health Communication 28: 814-821.

Siegrist, M., Stampfli, N. and Kastenholz, H. (2008). Consumers' willingness to buy functional foods. The influence of carrier, benefit and trust. Appetite 51: 526-529. 
Silayoi, P. and Speece, M. (2004). Packaging and purchase decisions: a focus group study on the impact of involvement level and time pressure. British Food Journal 106: 607-628.

Siró, I., Kápolna, E., Kápolna, B. and Lugasi, A. (2008). Functional food. Product development, marketing and consumer acceptance-A review. Appetite 51: 456-467.

Stein A.J. and Rodríguez-Cerezo E. (2008). Functional food in the European Union. Technical Report by the Joint Research Centre of the European Commission, EUR 23380 EN. Luxemburg: European Communities.

Szathvary, S. and Trestini, S. (2014). A hedonic analysis of nutrition and health claims on fruit beverage products. Journal of Agricultural Economics 65(2): 505-517.

Teisl, M. F. and Roe, B. (1998). The economics of labeling: An overview of issues for health and environmental disclosure. Agricultural and Resource Economics Review 27: 140-150.

Valls, J., Pasamontes, N., Pantaleón, A., Vinaixa, S., Vaqué, M., Soler, A., ... and Gómez, X. (2013). Prospects of functional foods/nutraceuticals and markets. Natural Products (pp.24912525). Springer Berlin Heidelberg.

Van Kleef, E., van Trijp, H.C.M. and Luning, P. (2005a). Functional foods: health claim-food product compatibility and the impact of health claim framing on consumer evaluation. Appetite 44: 299-308.

Van Kleef, E., van Trijp, H.C.M. and Luning, P. (2005b). Consumer research in the early stages of new product development: a critical review of methods and techniques. Food Quality and Preference 16: 181-201.

Verbeke, W., Scholderer, J. and Lähteenmäki, L. (2009). Consumer appeal of nutrition and health claims in three existing product concepts. Appetite 52: 684-692.

Wills, J. M., Storcksdieck genannt Bonsmann, S., Kolka, M., a Grunert, K. G. (2012). European consumers and health claims: attitudes, understanding and purchasing behaviour. Proceedings of the Nutrition Society 71(02): 229-236.

Wooldridge, J. M. (2002). Econometric Analysis of Cross Section and Panel Data. Cambridge, MA: MIT Press. 


\section{Chapter 4}

\section{Credence Attributes and the Quest for a Higher Price - a Hedonic Stochastic Frontier Approach.}

Alessandro Bonanno, Francesco Bimbo, Alfons Oude Lansink, Marco Costanigro, Rosaria Viscecchia. 


\title{
Credence Attributes and the Quest for a Higher Price - a Hedonic Stochastic Frontier Approach.
}

\begin{abstract}
Food manufacturers offering credence attributes, whose presence cannot be determined a priori, may fail to product differentiate and successfully escape from price competition, if asymmetric information impairs their ability to reach consumers with higher willingness to pay. In this article, we assess whether manufacturers carrying products with credence attributes in their portfolio are able to obtain higher prices using a large database of yogurt sales in Italy and a hedonic price model estimated using a Stochastic Frontier estimator. Results indicate that manufacturers offering more credence attributes in their portfolio have the ability to price their products systematically at higher levels.
\end{abstract}

Keywords: Hedonic Price Model; Credence Attributes; Stochastic Price Frontier

\section{Introduction}

Price dispersion occurs when the price of identical goods varies across suppliers and markets. The rationale for the existence of price dispersion has been explored at length in the economics literature: following the seminal work by Stigler (1961), several frameworks (inter alia Salop, 1977; Salop and Stiglitz, 1977; 1982; Burdett and Judd, 1983) showed that if information gathering for consumers is costly, asymmetric information may enable suppliers to price discriminate and more than one price equilibrium will emerge. ${ }^{1}$ Even though these theories were developed for homogenous product markets, a similar counterpart exists for differentiated product markets (e.g. Barron, Taylor and Umbeck, 2004; Lewis, 2008) where "local" competition and consumer search cost can lead to variation in differentiated products' prices even after differences in consumers' willingness to pay (WTP) for specific products are controlled for. In fact, information costliness can lead to systematic differences in price charged to consumers in differentiated product markets: Wildenbeest (2011), extending Armstrong and Vickers (2001) model of consumers search in a market with vertically differentiated products, shows that if information is costly some firms are able to charge systematically higher prices than their competitors.

Many differentiated food products can be characterized as credence goods, that is carrying credence attributes (i.e. "organic" or "natural" labels, healthy-claims, GMO-free etc.) whose

\footnotetext{
${ }^{1}$ Non-unique price equilibria can be sustained by numerous factors: asymmetric information (sellers have information on buyers' behaviour and on their reservation price), variation in product quality, or consumers' heterogeneity. Empirical applications studying price dispersion for homogenous products abound: Devine and Marion (1979) first empirical study of price dispersion in retail food prices in function of information; Borenstein and Rose (1994) analysis of competition in airline price dispersion; Sorensen (2000) study of price dispersion in the retail prescription drugs marker; Lach (2002) assessment of long-term price dispersion in retail prices of homogenous food products; Zhao's (2006) investigation of intra-brand, intra-store and intra-category price dispersion in several food and non-food grocery categories; and Richards, Hamilton and Allender (2016) study of search cost and price dispersion in UK online grocery.
} 
quality-level cannot be detected by consumers even after consumption (Nelson, 1970; Darby and Karni, 1973). Since quality signaling cannot be substantiated by consumers' experience, firms may struggle to develop reputations for credence quality traits, and consumers' heterogeneous beliefs about quality provision may lead to price dispersion. However, in presence of third party certifications and labeling regulations, asymmetric information can be reduced (e.g. Caswell and Padberg, 1992; Caswell and Mojduszka, 1996; Teisl and Roe, 1998) and, if the certification is probabilistically accurate, ${ }^{2}$ credence good markets can become experience good markets (McCluskey, 2000), and effective signaling can occur. If that is the case, then suppliers' reputation could come into play allowing sellers to target systematically consumers with higher WTP for products with similar features of their competitors'.

A strong link exists between the strength of a brand and the effectiveness of quality signalling, particularly in the context of credence attributes (Sporleder and Goldsmith, 2001). Some research indicates that signalling higher quality to consumers through credence attributes may lead to brand loyalty (e.g. Lassoued and Hobbs, 2015), whereas other (Bimbo, Bonanno and Viscecchia, 2016) shows that brand strength is linked to obtaining higher premiums for some types of health-claims in the Italian yogurt market. Furthermore, brand strength is considered as a quality signal in the wine market where both collective and private reputation can contribute to higher prices (e.g. Costanigro, McCluskey and Goemans, 2010). This would indicate that, at least potentially, a manufacturer carrying credence goods in its portfolio could benefit from improved brand image / reputation and improve their ability to target those consumers with a higher WTP. ${ }^{3}$ Adding products carrying credence attributes to their portfolio may also improve manufacturers' ability to charge higher prices through mechanisms other than reputation. Some studies suggest that product-line length ${ }^{4}$ can serve as a quality cue for consumers (Bergen, Draganska and Simonson, 2007), increasing consumers' brand loyalty, and ultimately allowing producers to charge higher prices (Draganska and Jain, 2005).

The central question addressed in this article pertains to whether credence labelling helps manufacturers to achieve higher prices, or if the presence of asymmetric information and consumers distrust hinders such ability. In other words, we seek answers to the question: do manufacturers adding products with credence attributes to their portfolio reach systematically consumers with higher WTP? Answering this research questions requires testing empirically whether the presence of products with credence attributes in a manufacturer's portfolio is related to the price of the different products sold, once other sources of price variation are controlled for, such as individual product characteristics, including the credence attributes

\footnotetext{
2 Bonroy and Constantatos (2008) show that, if consumers' beliefs regarding the firm that selling the high quality product are idiosyncratic, labels that restore full information improve welfare but cause a decline in firms' profits, and that imperfect labels can have adverse welfare effects. For a thorough discussion of the economics of labels and their effect on welfare see Bonroy and Constantatos (2015).

${ }^{3}$ For example, the literature summarized in Bimbo et al. (2017) shows that consumers with low interest in health show higher preference and acceptance of "health-enhancing" dairy products if these are offered by brands familiar to consumers.

4 "Product line length" refers to the total number of product options, or unique combinations of product features (e.g. type of flavour, fat content, consistency, health benefit) sold by a manufacturer.
} 
themselves, brands (which may embed "reputation" through other avenues, such as advertising etc.), as well as temporal and geographical variation.

An intuitively appealing framework to answer this question is the hedonic price model (Rosen, 1974) which maps a product price to the value of each embedded attribute (Lancaster, 1966) by tracing the envelope of the bid functions representing consumers' WTP for the different attributes and the offer functions representing producers technological constraints. However, the traditional hedonic price model is insufficient to address our problem for two reasons. First, in a traditional hedonic price model reputation effects are measured by estimating the implicit prices associated with specific labelling / brand attributes, affecting prices in an additive way (e.g. Costanigro, McCluskey and Goemans, 2010). We posit instead that increasing the number of credence attributes has a systematic effect on the shape of the entire hedonic price curve, and therefore we need to test for the existence of supra-additive effects. Second, the traditional hedonic price framework assumes that buyers and sellers are fully informed - that is every seller knows consumers' highest WTP and every buyer knows the sellers' lowest Willingness to Accept (WTA), which is not the case of markets with credence attributes. Recently, Kumbhakar and Parmeter (2010) extended Rosen's framework, allowing sellers' and buyers' characteristics to affect the shape of the hedonic price function, while at the same time relaxing the full information assumption and allowing for the coexistence of multiple hedonic functions, which depend upon the level of information that buyers and sellers possess. Kumbhakar and Parmeter (2010) model a portion of the unobservable price variation as a function of sellers' (buyers') features to capture the extent of information asymmetry which may prevent sellers (buyers) to fully exploit the highest (lowest) values of buyers' WTP (sellers' WTA) for a bundle of attributes available in a market, generating multiple price equilibria.

In this article we use Kumbhakar and Parmeter's (2010) modified hedonic price framework to test whether manufacturers investing in credence attributes are able to effectively signal product quality, and to price their products closer to the hedonic price curve characterized by consumers with the highest WTP. Thus, we assume a scenario where fully informed producers face imperfectly informed consumers, and test whether manufacturers' carrying a larger number of credence attributes are able to reach consumers with a higher WTP for their products. We estimate this "buyer's price frontier" (as defined by Kumbhakar and Parmeter (2010)) by means of a Stochastic Frontier (SF) estimator, which represents a novel approach in the context of hedonic price model applied to differentiated food products. ${ }^{5}$ For our empirical application we choose the Italian yogurt market, and a two-year scanner database of monthly yogurt sales at the regional level. We use different model specifications and variables to capture the number of credence attributes in a manufacturers' portfolio. We assume that the number of credence attributes affects the dispersion of manufacturers' prices below the hedonic price curve; given our hypothesis that firms using more credence attribute will charge

\footnotetext{
${ }^{5}$ SF instead, has been widely adopted to account for the sources of price heterogeneity in the housing (e.g. Kumbhakar and Parmeter, 2010; Carriazo, Ready and Shortle, 2013), lumber (Kalita et al., 2004), consumer electronics (Lee et al., 2008), and on-line airline ticket markets (Kamakura and Moon, 2009).
} 
(ceteris paribus) higher prices, we expect that more credence attributes are offered, the less dispersed (i.e. the closer to the buyer's price frontier) a firm's prices will be.

We choose the Italian yogurt market as a case study for different reasons. In general terms, the yogurt category is a fitting case study due to the high product heterogeneity and its oligopolistic nature (e.g. Villas Boas, 2007; Bonanno, 2013; Hovhannisyan and Bozic, 2013) which makes it an ideal candidate to test for the co-existence of different prices. Furthermore, Italian yogurt manufacturers have used different credence attributes to differentiate their products, from "Organic," "Natural," to a number of health claims (Bonanno, 2012) offering a large amount of heterogeneity that can results in different consumers' attitude towards them. For example, even though asymmetric information may cause consumers' distrust for products carrying health claims (e.g. Verbeke, 2005a; 2005b), resulting in some consumers' low WTP for products carrying these attributes, the presence of health claims in European markets has been subjected to a tight regulatory framework, the Reg. (EC) No.1924/2006. ${ }^{6}$ Therefore, if on the one hand, the stringency of the regulation may add to manufacturers' uncertainty about the profitability of their investment (e. g, Brookes, 2010) which may result in not all Italian yogurt manufacturers being able to use health claims as a means of product differentiation (e.g. Bimbo, Bonanno and Viscecchia, 2016), on the other hand, the high quality standard may be preferred by consumers with higher WTP and enable Italian yogurt manufacturers to exert higher prices.

The article proceeds as follows. First we present the empirical framework of our analysis, followed by a discussion of our model specification, the data used and the details of the estimation procedure adopted. The empirical results will be presented and discussed next. We conclude with a discussion of the limitations of the current work and avenues of future research.

\section{The Empirical Framework}

Our empirical framework starts from the traditional hedonic price model formalized by Rosen (1974). According to this framework, consumers choose products containing a utility maximizing bundle of attributes, subject to a budget constraint. Producers choose a profitmaximizing combination of attributes subject to the available technology embedded in the cost function. The first order conditions to each maximization problem leads to two families of indifference curves having product attributes as their arguments: consumers' bid and producers' offer functions. The bid function $\theta=\theta\left(x_{1}, x_{2}, \ldots, x_{k} ; u, y\right)$ represents the amount a consumer is willing to pay (WTP) for varying levels of the attribute vector $\mathbf{x}=\left(x_{1}, x_{2}, \ldots, x_{k}\right)$, holding utility, $u$, and income, $y$, constant. Analogous to the consumer's bid function, the seller's offer function $\varphi=\varphi\left(x_{1}, x_{2}, \ldots, x_{k} ; \pi\right)$ indicates the price that a firm is willing to accept (WTA) for selling a good with the characteristics vector $\mathbf{x}$ while maintaining the (fixed) profit

\footnotetext{
${ }^{6}$ The European Union's Regulation (EC) No.1924/2006 aims to reduce asymmetric information in food products markets and to guarantee that nutrition and health claims are truthful and understandable. Claims falling under article 13 are divided into "general function" claims (Article 13.1) and general function claims based on new and/or proprietary data (Article 13.5). Claims falling under Article 14, i.e. "risk reduction" claims deal with risk factor reduction in the development of a human disease. Research shows that "risk reduction" claims have higher market valuation than "general function" claims (i.e. Bimbo, Bonanno and Viscecchia 2016).
} 
$\pi$. Under the assumptions of perfect competition and full information, the double envelope traced by the points of tangency between bid and offer curves results in the hedonic price function $p(\mathbf{x})=p\left(x_{1}, x_{2}, \ldots, x_{k}\right)$, establishing a unique price conditional on $p^{0}(\mathbf{x})$; the two matched sets of bid/offer curves are labelled with the superscripts 1 and 2.

According to Rosen's model, the observed price of a product $j$ in market $m$ at time $t$, is a function of product attributes, plus an error term accounting for random shocks and unexplained product heterogeneity:

$$
P_{j m t}=f\left(\mathbf{x}_{j m t}, \boldsymbol{\beta}\right)+\varepsilon_{j m t}
$$

where the functional form $f(\bullet)$ is to be determined empirically and $\boldsymbol{\beta}$ is a conforming vector of parameters to be estimated (Costanigro and McCluskey, 2011).

Kumbhakar and Parmeter (2010) modify the classical hedonic framework to relax the full information assumption, allowing for multiple price equilibriums to co-exist when information is imperfect or asymmetric. In their model, consumers with the highest WTP for a certain attribute level identify the upper boundaries of the attainable market prices $p^{H I G H}(\mathbf{x})$ (buyer's price frontier) while producers with the lowest WTA trace the lower bounds $p^{L O W}(\mathbf{x})$ (seller's price frontier) as in figure 1 in Appendix I, middle panel. Lacking full information, transactions can occur anywhere in-between the two lines, with the two polar cases of fully informed producers matched to information deficient consumers (offer and bid curves, respectively, carrying the superscripts 3 and 4) or fully informed buyers matched with information-deficient sellers (offer and bid curves, respectively, showing superscripts 5 and 6 ). Our analysis investigates the case of fully informed producers and imperfectly informed consumers; that is, we focus on the ability of manufacturers to reach $p^{H I G H}(\mathbf{x})$, depicted in the right-hand panel of figure 1 in Appendix I.

Thus, equation (1), which treats the error term as a nuisance parameter, is insufficient to model systematic departures from the hedonic price function. Following Kumbhakar and Parmeter (2010) we account for buyers with the highest WTPs or sellers with the lowest WTAs by separating the error term in equation (1) in three components:

$$
P_{j m t}=f\left(\mathbf{x}_{j m t}, \boldsymbol{\beta}\right)+\varepsilon=f\left(\mathbf{x}_{j m t}, \boldsymbol{\beta}\right)+v+w-u .
$$

Equation (2) shows that that the unobserved portion of the price of product $\mathrm{j}$ in market $\mathrm{m}$ at time $\mathrm{t}$ is constituted of $\mathrm{v}$, a random noise component, and $\mathrm{u}$ and $\mathrm{w}$, which are two one-sided (half positive) errors. The term $\mathrm{w}(\mathrm{w} \geq 0)$ represents the cost incurred by consumers for not being fully informed and/or for not being able to assess the "true" value of a given product. The term $u$ instead $(u \geq 0)$, is the loss that a seller may incur for not being able to target those consumers with the highest WTP for a product containing a given bundle of attributes. Thus, in a market characterized by asymmetric information, the random term $\varepsilon$ embodies two different measures of price inefficiencies, one from the consumers' standpoint, $(w)$ and another from the producer's $(u)$. Note that under such specification $E(\varepsilon)$ may be non-zero, even when $E(v)=0$. In the presence of either inefficiencies on both sides (Kumbhakar and 
Parmeter, 2010), or on one side of the market only, (e.g. Kamakura and Moon, 2009; Carriazo, Ready and Shortle, 2013) the structure of the half-positive errors should be appropriately specified.

As illustrated above, in this application we assume $w=0$ and parameterize the variance of the one-sided error $u, \sigma_{u}^{2}$ to capture the determinants of manufacturer's inability to reach consumers with the highest WTP. Following previous literature (e.g. Carriazo, Ready and Shortle, 2013) the following specification of the variance of the half-positive error $u$ is assumed:

$$
\sigma_{u}^{2}=\exp \left(\lambda^{\prime} \mathbf{z}\right)
$$

where the vector $\mathbf{z}$ contains variables affecting the ability of manufacturers to charge prices closer to the buyer's price frontier (illustrated below), and $\lambda$ is a conformable vector of parameters.

\section{Model Specification, Data and Estimation}

\section{Model Specification}

For our specification of equation (2), $\mathbf{x}$ is partitioned into five vectors, i.e. $\mathbf{x}^{C A}, \mathbf{x}^{N O C}, \mathbf{x}^{P}, \mathbf{x}^{R}$ and $\mathbf{x}^{\boldsymbol{B}}$ (product, market and time subscripts are omitted for brevity). $\mathbf{x}^{C A}$ represents a vector of product characteristics capturing credence attributes $(C A)$ indexed by $h(h=1, \ldots, H) ; \mathbf{x}^{N O C}$ includes non-credence $(N O C)$ product characteristics, indexed by $l(l=1, \ldots, L) . \mathbf{x}^{P}$ and $\mathbf{x}^{R}$ include package and retail characteristics and are indexed by $(p=1, \ldots, P)$ and $(r=1, \ldots, R)$, respectively. Last, $\mathbf{x}^{\boldsymbol{B}}$ is a vector of brand-specific indicator variables indexed by $b(b=1, \ldots, B)$.

Following previous literature (and for simplicity), equation (1) is estimated using a single equation approach (inter alia, Costanigro, McCluskey and Mittelhammer, 2007; Costanigro, McCluskey and Goemans, 2010; Bimbo, Bonanno and Viscecchia, 2016). For the empirical specification of equation (1) we use a log-linear functional form:

$$
\text { (4) } \begin{aligned}
\ln (P)_{j m t}= & \alpha+\sum_{h=1}^{H} \alpha_{h} x_{h m t}^{C A}+\sum_{l=1}^{L} \beta_{t} x_{l m t}^{N O C}+\sum_{p=1}^{P} \delta_{p} x_{p m t}^{P}+\sum_{r=1}^{R} \gamma_{r} x_{r m t}^{R}+ \\
& \sum_{b=1}^{B} \kappa_{b} x_{b m t}^{B}+\sum_{m=1}^{M} \theta_{m}^{M} d_{m}+\sum_{t=1}^{T} \theta_{t}^{T} d_{t}+\varepsilon_{j m t}
\end{aligned}
$$

where we also control for spatial- and time-variation in prices by means of a vector of $M$ market-level (region) and $T$ time (month) indicators, $d_{m}$ and $d_{t}$, respectively. The $\alpha_{s}, \beta_{s}, \delta_{s}, \gamma_{s}$ and $\kappa_{s}$ are parameters to be estimated capturing, respectively, the market valuation of credence attributes, non-credence attributes, packaging, retail-specific variables and brands, and $\varepsilon_{j m t}$ is an error term whose properties are discussed above. 
We use four different specifications of equation (3)

$$
\begin{aligned}
\sigma_{u}^{2} & =\exp \left(\lambda_{0}+\sum_{k=1}^{4} \lambda_{k}^{H C} H C_{k}\right) \\
\sigma_{u}^{2} & =\exp \left(\lambda_{0}+\sum_{i=1}^{4} \lambda_{i}^{C R} C R_{i}\right) \\
\sigma_{u}^{2} & =\exp \left(\lambda_{0}+\lambda^{N H C} N H C+\lambda^{\text {NHCItems }} N H C^{*} \text { Items }\right) \\
\sigma_{u}^{2} & =\exp \left(\lambda_{0}+\lambda^{N C R} N C R+\lambda^{\text {NCRItems }} N C R^{*} \text { Items }\right)
\end{aligned}
$$

where $H C_{k}$ is an indicator variable capturing whether a manufacturer has in its portfolio a given number of products with functional / health claims $\{k=1, \ldots, 4\} ; C R_{i}$ is an indicator variable capturing whether a manufacturer produces a given number of products with credence attributes $\{i=1, \ldots, 4\} N C R$ is a variable capturing the number of different credence attributes offered by a manufacturer in its portfolio; $N H C$ takes values equal to the number of functional / health claims in a manufacturer's portfolio. Last, Items is the average number of product items sold in the stores by each manufacturer.

Our hypothesis is that by offering a larger number of credence attributes, manufacturers can increase their ability to price their products closer to the buyer's price frontier; thus, we expect $\sigma_{u}^{2}$ to decline with the number of credence attributes produced. In other words, for specifications 5a and 5b, we expect: 1 ) the signs of the $\lambda_{i}^{C R}$ and $\lambda_{k}^{H C}$ parameters to be negative, and 2) $\left|\lambda_{i}^{C R}\right|<\left|\lambda_{i+1}^{C R}\right|$ and $\left|\lambda_{k}^{H C}\right|<\left|\lambda_{k+1}^{H C}\right|$ for $i=\{1, \ldots, 3\}$ and $k=\{1, \ldots, 3\}$. If these conditions are met, they will provide empirical support to the hypothesis that manufacturers' producing more products with credence attributes can systematically reach consumers with higher WTP. Similarly, for specifications $5 \mathrm{c}$ and $5 \mathrm{~d}$ we expect $\lambda^{N H C}<0$ and $\lambda^{N C R}<0$. Also, in line with Draganska and Jain (2005) result that longer product-line length can increase brandloyalty and charge higher prices we expect negative signs for $\lambda^{\text {NHCItem }}$ and $\lambda^{\text {NCRItems }}$. As a

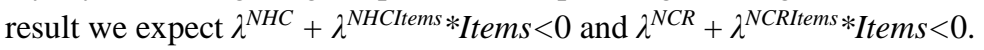

\section{Data and Variables}

The data used in the estimation come from SymphonyIRI Group and contain monthly sales information (point-of-sale) of yogurts in the whole Italian market (17 IRI regions ${ }^{7}$ ) encompassing a 25-month period between November 29, 2010, and December 31, 2012. The data contains information on volume sold and value of sales, price (EUR/l), percentage of store selling each product and number of items sold in the stores. The IRI data separate yogurts with health claims from others, and also provide detailed information on vendors,

\footnotetext{
${ }^{7}$ Although the Italian regions are 20, SymphonyIRI groups data from Piedmont and Aosta Valley, Abruzzo and Molise, Basilicata and Calabria, resulting in 17 "IRI regions".
} 
brands, flavours, fat content, drinkability, whether a yogurt is organic or labelled as "natural", whether it is targeted to kids and a series of information related to packaging and a product's distribution. The information contained in the database was cross-validated using information retrieved from manufacturers' websites, products' front-of-package, and nutritional labels.

The vector of credence attributes $\mathbf{x}^{C A}$, contains six indicator variables, one for each health claim, one for "organic" and one for "natural." Circa 30\% of products in our sample have health claims, which are classified in four classes: "enhancing the immune system" (Immunity, $11.4 \%$ of the sample); "sustaining bowel regularity" (Regularity, $14.4 \%$ ); "lowering or managing cholesterol level" (Chol. Reduction, 3.4\%); and "supporting bone health" (Bone Health, 1.1\%). Approximately $9 \%$ of the products in our sample are sold as "organic" (Organic, 7.4\%) or "natural" (Natural, 1.5\%).

The vector of non-credence attributes $\mathbf{x}^{\text {NOC }}$ includes seven indicator variables capturing the products' flavours (Plain and Fruit Flavours), fat content (Low Fat and Fat Free), whether the product is drinkable (Drinkable), added with fibre (Fibre) and lactose free (Lactose Free). The vector of packaging variables $\mathbf{x}^{\boldsymbol{P}}$ includes four indicator variables capturing: whether the product was sold in a glass jar (Glass Pack), whether it has an additional compartment with cereals and/or chocolate (Two Compartments), if it was sold in packages between 300 and $500 \mathrm{ml}$ (Medium Pack) or larger than $500 \mathrm{ml}$ (Large Pack). The vector of retail specific variables $\mathbf{x}^{\boldsymbol{R}}$ contain three variables: the average number of product items sold in the stores (Items), the percentage of products sold under promotion (\% Sales Prom), and the percentage of outlets offering a product conditional on it being available in a given store, i.e. the average weighted distribution $(A W D){ }^{8}$

After eliminating products with local distribution and products whose attribute profile could not be verified, ${ }^{9}$ the final database consisted of 54,386 observations including 220 products with unique combinations of attributes, encompassing 59 brands sold by 13 vendors (i.e. manufacturers). Summary statistics of variables included in the model are reported in Table 1.

Below we present some descriptive tests statistics to indicate whether the distribution of prices in our data differ conditional on the presence of credence attributes (the four health claims discussed above, organic and natural). The average price of yogurts without credence attribute is $3.897 \mathrm{EUR} / \mathrm{l}$, for a standard deviation of 1.282 , whereas that of products with credence attributes is $4.855 \mathrm{EUR} / \mathrm{l}$, standard deviation of 1.275 , for coefficients of variation of 0.329 and 0.262 , respectively. These values indicate that the price distribution of yogurts without credence attributes is $25.3 \%$ more dispersed than that of products with credence attributes. Using the natural $\log$ of prices, the difference in coefficients of variation is even more marked, approximately $50 \%$, with values of, respectively, 0.257 for products without (mean 1.305, standard deviation 0.335 ) and 0.167 for products with credence attributes (mean

\footnotetext{
${ }^{8}$ Even though none of the retail variables are, strictly speaking, "product attributes," their inclusion is necessary since products' store presence can be used strategically by retailers and can affect pricing strategies; thus, their omission could lead to omitted variable bias in our results.

${ }^{9}$ Private labels, whose attributes could not be verified are not included in the analysis along with products classified as "other functionality". Other products excluded from the analysis were yogurts made with milk different from cow milk and yogurts for kids. Also, we eliminated those vendors that were absent in one or more Italian region.
} 
Chapter 4

1.547, standard deviation 0.258). These findings are supported by the results of KolmogorovSmirnov tests for equality in distribution: the null hypothesis of the price (log-price) distributions of yogurts with and without credence attributes being statistically equal, can be rejected at the $1 \%$ probability level. ${ }^{10}$

Table 1. Descriptive statistic $(\mathrm{N}=54,386)$

\begin{tabular}{lrrrr}
\hline \multicolumn{1}{c}{ Variable } & Mean & Std.Dev. & Min & Max \\
\hline Price & 4.255 & 1.361 & 0.890 & 15 \\
Credence Attributes & & & & \\
$\quad$ Chol. Reduction & 0.034 & 0.181 & 0 & 1 \\
$\quad$ Immunity & 0.114 & 0.318 & 0 & 1 \\
$\quad$ Regularity & 0.144 & 0.351 & 0 & 1 \\
Bone Health & 0.011 & 0.107 & 0 & 1 \\
Natural & 0.015 & 0.120 & 0 & 1 \\
$\quad$ Organic & 0.074 & 0.261 & 0 & 1 \\
Non-Credence Attributes & & & & \\
Plain & 0.294 & 0.455 & 0 & 1 \\
Fruit Flavours & 0.502 & 0.500 & 0 & 1 \\
Others Flavours (excluded) & 0.205 & 0.404 & 0 & 1 \\
Regular (Excluded) & 0.482 & 0.500 & 0 & 1 \\
Low Fat & 0.261 & 0.439 & 0 & 1 \\
Fat Free & 0.257 & 0.437 & 0 & 1 \\
Lactose Free & 0.020 & 0.139 & 0 & 1 \\
Drinkable & 0.216 & 0.412 & 0 & 1 \\
Fibre & 0.060 & 0.238 & 0 & 1 \\
Packaging Characteristics & & & & \\
Glass Pack & 0.022 & 0.146 & 0 & 1 \\
Two Compartments & 0.096 & 0.294 & 0 & 1 \\
Regular Pack (excluded) & 0.751 & 0.432 & 0 & 1 \\
Medium Pack & 0.249 & 0.433 & 0 & 1 \\
Large Pack & 0.001 & 0.036 & 0 & 1 \\
Retail Characteristics & & & & \\
Num Items & 2.990 & 3.011 & 0 & 29 \\
\% Sales Prom & 0.147 & 1.438 & 0 & 100 \\
AWD & & & & 100 \\
\hline
\end{tabular}

The difference in distribution can also be visualized in Box-Plots and kernel density plots presented in Figure 2 in Appendix I. The evidence that prices and their natural logs are less dispersed for products with credence attributes than for those without, lends preliminary support to our hypothesis that the presence of credence attributes may allow manufacturers to consistently price at higher levels, closer to the buyer's price frontier.

\footnotetext{
${ }^{10}$ The value of the combined D-statistics for the Kolmogorov-Smirnov test is of 0.0356 , for a $p$-value of 0.000 . Similar results are obtained if natural $\log$ is used instead of price levels.
} 


\section{Estimation}

Following the notation in Kumbhakar and Lovell (2000) our hedonic price model can be rewritten as $\ln P_{j m t}=f(\mathbf{x}, \boldsymbol{\beta})+\varepsilon_{j m t}=f(\mathbf{x}, \boldsymbol{\beta})+v_{j m t}-u_{j m t} ; \quad$ where $\quad v \sim N\left(0, \sigma_{V}^{2}\right) \quad$ and $u \sim N^{+}\left(0, \sigma_{u}^{2}\right)$ its parameters estimated by maximising the likelihood function,

$$
L=\prod_{j} \prod_{m} \prod_{t} \frac{2}{\sigma} \phi\left(\frac{\varepsilon_{j m t}}{\sigma}\right) \Phi\left(\delta \frac{\varepsilon_{j m t}}{\sigma}\right) ;
$$

where $\sigma^{2}=\sigma_{u}^{2}+\sigma_{v}^{2} ; \quad \delta=\frac{\sigma_{u}}{\sigma_{v}}, \phi(\bullet)$ and $\Phi(\bullet)$ are the standard normal $p d f$ and $C D F$, respectively. Wald tests on the estimated standard deviation of the half-normal error $\sigma_{u}$ can be performed to indicate whether the data support the use of SF regression: in the context of our analysis, failing to reject the null of $\sigma_{u}=0$, indicates no systematic departure from the estimated buyer's price frontier; also given our specifications of $\sigma_{u}$ failing to reject $\sigma_{u}=0$, indicates that the presence of products carrying credence attributes do not effect in any a supplier's ability to reach higher prices. Alternative specifications of $\sigma_{u}^{2}$ consistent with equations $5 \mathrm{a}$ through $5 \mathrm{e}$ are estimated to test whether the presence of more products containing credence attributes results in prices closer to the buyers' price frontier. Data manipulation and estimation was performed using STATA version 13.

\section{Empirical Results}

Table 2 presents the estimated parameters obtained from the different specifications of the hedonic model estimated using SF including a half-normal one-sided error, with homoscedastic variance, which is referred to below as "baseline SF specification" as well as the heteroscedastic specifications of $\sigma_{u}^{2}$ consistent with equations $5 \mathrm{a}$ through $5 \mathrm{~d}$. The estimated coefficients associated with "retail-specific variables" as well as brand-level fixedeffects and monthly and regional indicator variables are omitted for brevity but available upon request.

The first result to be noted is that the estimated coefficients are relatively robust for different specification of the variance of the half-normal error $u$, as one can note comparing the estimated coefficients in the top panel of Table 2. Also, across all specifications of $\sigma_{u}^{2}$, we reject the null hypothesis of $\sigma_{u}^{2}=0$, supporting the existence of systematic departure from the buyer's price frontiers. In particular, the estimated ratio $\sigma_{u} / \sigma_{v}$ from the Baseline SF specification shows that the standard deviation of the half normal component of the error term is twice as large as that of the idiosyncratic residual, indicating that price inefficiencies lead to twice as much price variation compared to the dispersion of prices above and below the estimated hedonic curve. 
Chapter 4

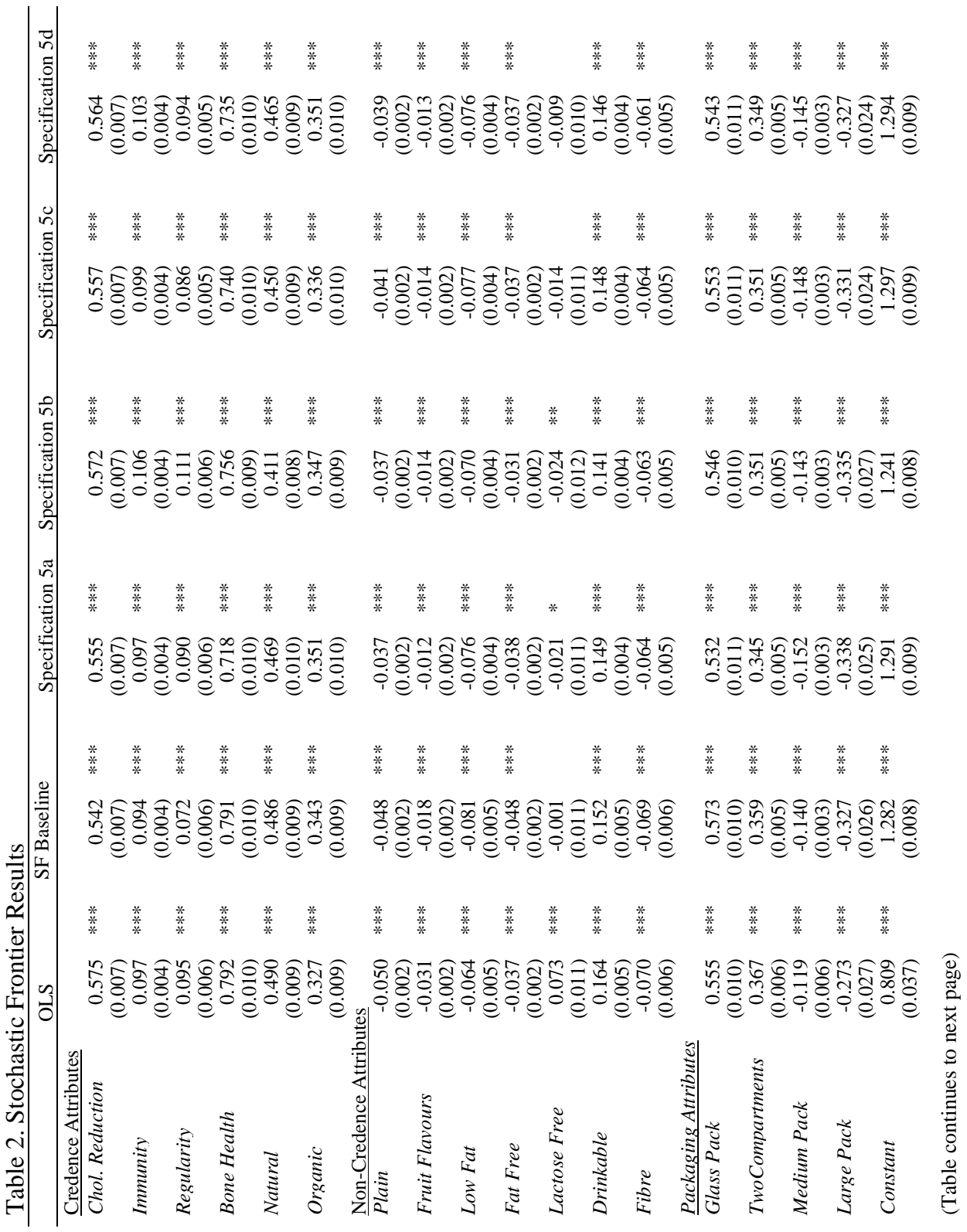


Chapter 4

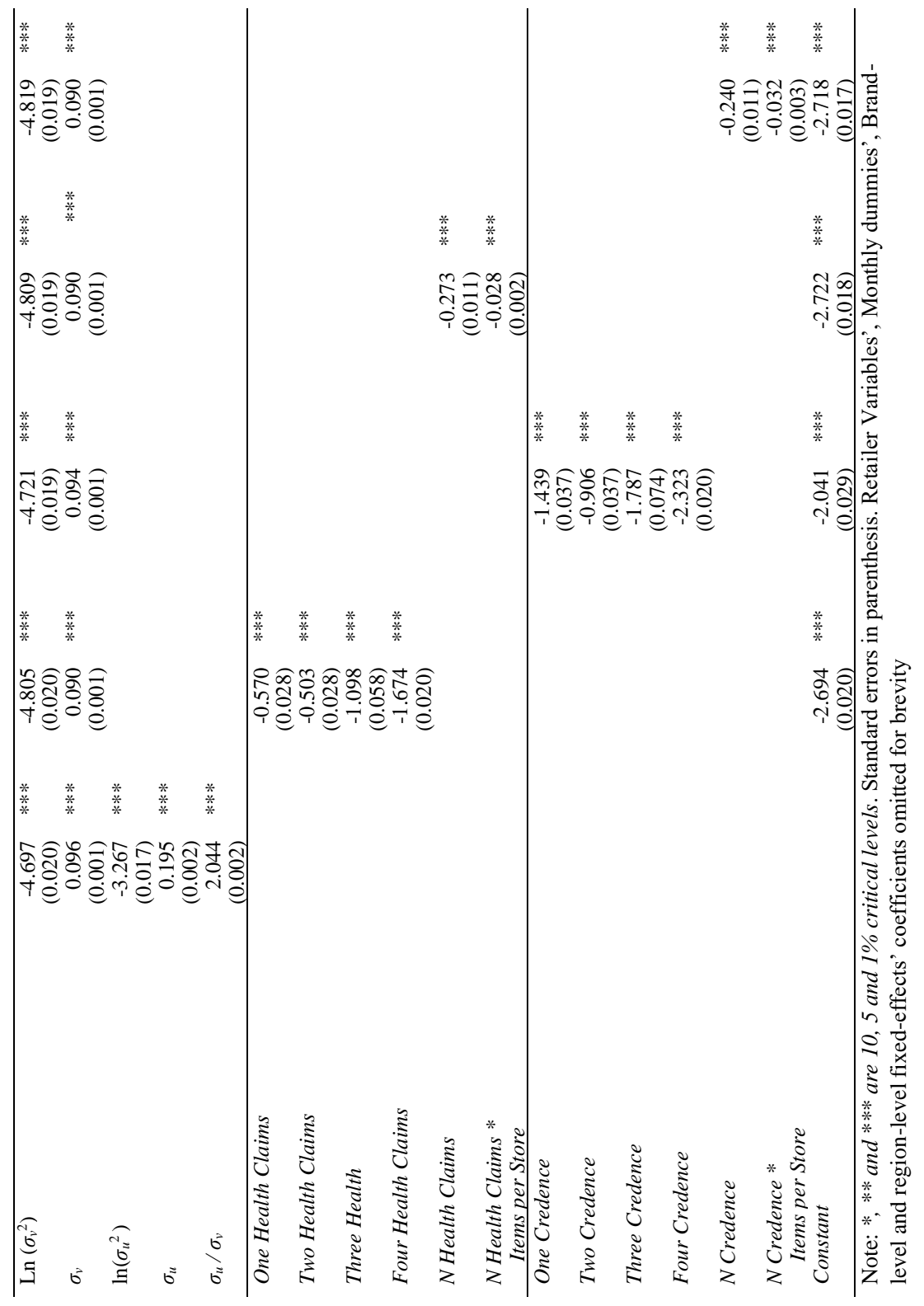


Focusing on the estimated parameters of the hedonic price function, credence attributes, four of them have the largest effect on price: claims supporting bone health (Bone Health) whose coefficients range from 0.718 to 0.791 (Specification 5a, and Baseline specification, respectively); Natural, with coefficients between 0.411 (Specification 5b) and 0.486 (Baseline specification); lowering blood cholesterol level (Chol. Reduction), coefficients of 0.542 (Baseline specification) and 0.572 (Specification 5b); and Organic, from 0.336 (Specification 5c) to 0.343 (Baseline specification). The coefficients for Immunity and Regularity, although statistically significant, are considerably smaller than those of the other four credence attributes. Most non-credence attributes do not contribute positively to prices, with the exception of Drinkable, whose coefficient varies between 0.141 (Specification 5b) and 0.152 (Baseline specification), Glass Pack and Two Compartments which results in coefficients ranging from 0.532 (Specification 5a) and 0.573 (Baseline). Last, as one may expect, the higher the packaging sizes, the higher the price discount. Thus, overall, these results point to credence attributes, drinkable texture, glass packaging and the presence of a second ingredient compartment, as sources of effective product differentiation.

The results presented in the bottom section of Table 2, show the estimated $\log \left(\sigma_{u}^{2}\right)$ and its components for the baseline SF model specification, as well as its constant terms for the specifications $5 \mathrm{a}-5 \mathrm{~d}$ to be statistically different from zero, which indicates that the data support the existence of systematic departures from the buyers' price frontier. Also, as hypothesized, all coefficients for the shifters of $\sigma_{u}^{2}$ in equations $5 \mathrm{a}-5 \mathrm{~d}$ are negative. The magnitude of the coefficients for specifications 5a-5b grow larger with the number of credence (or functional) attributes carried in a manufacturer's portfolio, with the exception of the coefficients of One Health Claim and Two Health Claims in specifications 5a, which are not statistically different from one another, and that for One Health Claim being larger in magnitude and statistically different than Two Health Claims in specification 5b. Also, in specifications $5 \mathrm{c}$ and $5 \mathrm{~d}$, the estimated coefficients associated with the actual number of functional / health claims, credence attributes, as well as those obtained for their interactions with the number of product items sold in the store are both negative and statistically significant.

The estimated coefficients reported in the bottom panel of Table 3 are used to calculate the variance of the half-normal error as in equations $5 \mathrm{a}-5 \mathrm{~d}$, and are reported in Table 4. Confirming our hypothesis that the larger the number of credence attributes, the higher the prices, the estimated $\sigma_{u}^{2}$ is the highest for those manufacturers that do not include in their portfolio health claims (5a, for a variance of 0.068 and 5c, for a variance of 0.066 ) or credence attributes in general (specifications $5 \mathrm{~b}$, and $5 \mathrm{~d}$ with variances of 0.130 and 0.066 , respectively), and as the number of health claims, or credence attributes in general increases, the dispersion of prices from the buyer's price frontier decreases. Although we find that, in specification 5a (5b), having one or two health claims (credence attributes), does not result in a different level of dispersion from the buyers' price frontier, Italian yogurt manufacturers 
with three or four health claims (credence attributes) in their product portfolios can price their product much closer to the price frontier, with estimated $\sigma_{u}^{2}$ of 0.023 and 0.013 , respectively.

Table 3. Decomposition of Half-Normal Error Variance $\left(\sigma_{u}^{2}\right)-$ Specifications $5 a-5 d$

\begin{tabular}{|c|c|c|c|c|c|c|c|}
\hline & Specificat & on $5 \mathrm{a}$ & Specificat & ion $5 b$ & Specifica & tion $5 c$ & Specification $5 \mathrm{~d}$ \\
\hline No Health Claims & $\begin{array}{r}0.068 \\
(0.001)\end{array}$ & $* * *$ & & & $\begin{array}{r}0.066 \\
(0.001)\end{array}$ & $* * *$ & \\
\hline No Credence & & & $\begin{array}{r}0.130 \\
(0.004)\end{array}$ & $* * *$ & & & $\begin{array}{l}0.066 \quad * * * \\
(0.001)\end{array}$ \\
\hline One Health Claim & $\begin{array}{r}0.038 \\
(0.001)\end{array}$ & $* * *$ & & & $\begin{array}{r}0.046 \\
(0.001)\end{array}$ & $* * *$ & \\
\hline Two Health Claims & $\begin{array}{r}0.041 \\
(0.001)\end{array}$ & $* * *$ & & & $\begin{array}{r}0.032 \\
(0.001)\end{array}$ & $* * *$ & \\
\hline Three Health & $\begin{array}{r}0.023 \\
(0.001)\end{array}$ & $* * *$ & & & $\begin{array}{r}0.025 \\
(0.001)\end{array}$ & $* * *$ & \\
\hline Four Health & $\begin{array}{r}0.013 \\
(0.001)\end{array}$ & $* * *$ & & & $\begin{array}{r}0.014 \\
(0.001)\end{array}$ & $* * *$ & \\
\hline One Credence & & & $\begin{array}{r}0.031 \\
(0.001)\end{array}$ & $* * *$ & & & $\begin{array}{l}0.047 \quad * * * \\
(0.001)\end{array}$ \\
\hline Two Credence & & & $\begin{array}{r}0.053 \\
(0.001)\end{array}$ & $* * *$ & & & $\begin{array}{l}0.034 \text { *** } \\
(0.001)\end{array}$ \\
\hline Three Credence & & & $\begin{array}{r}0.022 \\
(0.001)\end{array}$ & $* * *$ & & & $\begin{array}{l}0.026 * * * \\
(0.001)\end{array}$ \\
\hline Four Credence & & & $\begin{array}{r}0.013 \\
(0.001)\end{array}$ & $* * *$ & & & $\begin{array}{l}0.015 * * * \\
(0.001)\end{array}$ \\
\hline
\end{tabular}

Note: $*, * *$ and $* * *$ are 10,5 and $1 \%$ critical levels. Standard errors in parenthesis.

The estimated $\sigma_{u}^{2}$ for specifications $5 \mathrm{c}$ and $5 \mathrm{~d}$ also decreases with the number of credence and health claims/functional attributes, showing magnitudes in line with those obtained for the other specifications. Also, in spite of the magnitudes of the estimated $\sigma_{u}^{2}$ in absence of credence attributes/health claims differ slightly across specifications (from 0.066 for specifications $5 \mathrm{c}$ and $5 \mathrm{~d}$ to 0.130 for specification $5 \mathrm{~b}$ ), our results appear robust as the magnitude of the estimated $\sigma_{u}^{2}$ for the larger number of credence attributes/health claims vary between 0.013 and 0.015 . The percentage implicit prices associated with different product attributes, and across model specifications, are presented in table 4, and are obtained using Kennedy (1981) adjustment. An interesting result is that, in most cases, the implicit prices calculated from the SF estimates are smaller than those obtained from the OLS ones, or in the case of those resulting in a discount (such as some of the non-credence attributes) show higher magnitudes, to indicate a larger discount.

All credence attributes affect positively yogurts' prices, with claims supporting bone health (Bone Health) and lowering cholesterol level in the blood (Chol. Reduction) outperforming all other health claims as well as Natural and Organic. Specifically, the health claims with the lowest contribution to prices are "Regulating the intestinal tract" (Regularity) with estimated implicit prices ranging from $7.47 \%$ (Baseline specification) and $11.69 \%$ (Specification $5 \mathrm{~b}$ ), 
and "Supporting the immune system" (Immunity) whose implicit price varies between $9.81 \%$ (Baseline specification) and $11.23 \%$ (5b). These modest implicit prices may be due to the limited level of differentiation supported by these attributes, as products carrying them have been in the market for several years (Bimbo, Bonanno and Viscecchia, 2106). The implicit price of Cholesterol Reduction claims varies from $71.92 \%$ to $77.21 \%$ (the OLS one in this case is higher at $77.71 \%$ ) while that of Bone Health exceeds $120 \%$ (120.77 for the OLS and 120.62 for the baseline SF model). The high market valuations of Cholesterol Reduction and Bone Health are in line with previous studies (Ares and Gámbaro, 2007; Siegrist et al., 2008; Bimbo, Bonanno, and Viscecchia, 2016). Organic and Natural, which are not associated with specific benefits for consumers, show percentage implicit prices ranging from $39.90 \%$ (Specification 5c) to $42.09 \%$ (Specification 5d) and from $50.87 \%$ (5b) to $63.24 \%$ (Baseline), respectively, according to SF estimates. The OLS implicit prices for these attributes are, respectively $38.67 \%$ and $63.22 \%$, indicating that, at the buyer's price frontier, the organic attribute is likely to lead to higher premiums, while "natural" to lower ones. Overall, these implicit prices are likely the result of consumers' willingness to pay for features having a "halo effect" (Schuldt and Schwarz, 2010; Schuldt, 2013), as products labelled "Organic" and "Natural" are often perceived as healthier than regular ones (e.g. Lodorfos and Dennis, 2008; Schuldt and Schwarz, 2010).

Table 4. Percentage Implicit Prices - alternative model specifications.

\begin{tabular}{|c|c|c|c|c|c|c|}
\hline Variables & OLS & $\begin{array}{c}\text { SF } \\
\text { Baseline }\end{array}$ & $\begin{array}{c}\text { Spec. } \\
5 \mathrm{a} \\
\end{array}$ & $\begin{array}{c}\text { Spec. } \\
5 \mathrm{~b}\end{array}$ & $\begin{array}{c}\text { Spec. } \\
5 \mathrm{c}\end{array}$ & $\begin{array}{c}\text { Spec. } \\
5 \mathrm{~d}\end{array}$ \\
\hline \multicolumn{7}{|l|}{ Credence Attributes } \\
\hline Chol. Reduction & 77.71 & 71.92 & 74.18 & 77.21 & 74.46 & 75.74 \\
\hline Immunity & 10.19 & 9.81 & 10.18 & 11.23 & 10.44 & 10.82 \\
\hline Regularity & 9.96 & 7.47 & 9.40 & 11.69 & 8.94 & 9.88 \\
\hline Bone Health & 120.77 & 120.62 & 104.98 & 112.95 & 109.68 & 108.57 \\
\hline Natural & 63.23 & 62.64 & 59.86 & 50.87 & 56.76 & 59.18 \\
\hline Organic & 38.68 & 40.95 & 42.06 & 41.47 & 39.90 & 42.09 \\
\hline \multicolumn{7}{|c|}{ Non-Credence Attributes } \\
\hline Plain & -4.88 & -4.73 & -3.66 & -3.62 & -4.04 & -3.83 \\
\hline Fruit Flavours & -3.05 & -1.78 & -1.20 & -1.37 & -1.37 & -1.25 \\
\hline Low Fat & -6.20 & -7.77 & -7.28 & -6.73 & -7.41 & -7.33 \\
\hline Fat Free & -3.63 & -4.65 & -3.76 & -3.04 & -3.67 & -3.65 \\
\hline Lactose Free & 7.57 & -0.14 & -2.05 & -2.34 & -1.36 & -0.90 \\
\hline Drinkable & 17.82 & 16.45 & 16.06 & 15.19 & 15.92 & 15.76 \\
\hline Fibre & -6.76 & -6.70 & -6.23 & -6.11 & -6.18 & -5.89 \\
\hline \multicolumn{7}{|l|}{ Packaging Attributes } \\
\hline Glass Pack & 74.19 & 77.29 & 70.18 & 72.56 & 73.92 & 72.10 \\
\hline Two Compartments & 44.34 & 43.23 & 41.16 & 42.05 & 41.98 & 41.76 \\
\hline Medium Pack & -11.22 & -13.08 & -14.08 & -13.36 & -13.73 & -13.52 \\
\hline Large Pack & -23.92 & -27.94 & -28.73 & -28.50 & -28.18 & -27.93 \\
\hline AWD & -0.10 & -0.12 & -0.11 & -0.11 & -0.11 & -0.11 \\
\hline
\end{tabular}

Note: the percentage implicit prices are calculated using Kennedy (1981) adjustments 
The estimated coefficients for the non-credence attributes are reported in the second part of Table 4. Plain and Fruit flavours show negative implicit prices: the estimated SF implicit prices of Plain range from -3.62 to $-4.73 \%$ whereas those of Fruit flavours from -1.20 to $1.78 \%$. As the OLS implicit prices for both these attributes are still negative, but show a larger magnitude, price discounts will be more marked further away from the buyer's price frontier (i.e. at lower price levels). These results support other findings that "flavour" may not be an effective price differentiation tool in the Italian yogurt market (e.g. Bonanno, 2013). The estimated implicit prices for Low Fat and Fat Free are also negative; however, the magnitude of those obtained from SF coefficients are larger than the OLS ones, to indicate that attributes related to reduced-fat content generate a higher discount at the buyer's price frontier than at lower price levels. Overall, fat content does not seem to be a source of product differentiation in the Italian yogurt market (Carlucci et al., 2013; Bonanno, 2013).

The estimated implicit price of Drinkable obtained from SF coefficients vary between $15.19 \%$ and $16.45 \%$, lower than the OLS one $(17.82 \%)$; these result seem in line with previous findings that Italian yogurt consumers prefer drinkable yogurts to regular ones (Bonanno 2013), while also suggesting that this attribute is slightly less important for consumers with the highest WTP. An interesting result is that for Lactose Free; the OLS implicit price is positive $(7.57 \%$ ) whereas the SF ones are negative, ranging from values close to zero, $-0.14 \%$ (Baseline specification) to $-2.34 \%$ (Specification $5 \mathrm{~b}$ ). These results suggest that while this feature may help reaching higher prices for products targeting consumers with lower WTP, its ability to contribute to product differentiation is limited at higher prices. This could be the result of consumers with higher WTP substituting lactose free dairy products with specific medicines that allow them to consume regular dairy products. Presence of added fibre (Fibre) affects yogurt's price negatively; the SF implicit prices vary from $-5.89 \%$ (Specification $5 \mathrm{~d}$ ) to $-6.709 \%$ (Baseline), the OLS one is $-6.76 \%$; these results suggest that adding fibre to yogurts may result in a price reduction regardless on its price level. This finding is supported by other studies highlighting that consumers can be sceptical of features "unnaturally" or artificially added to a product (Krutulyte et al., 2010; Annunziata and Vecchio, 2013).

The estimated implicit prices for the packaging characteristics are shown in the bottom section of Table 4. Glass packaging (Glass Pack) and the presence of a two-compartment package (Two Compartments) affect yogurts' price positively, the former resulting in implicit prices ranging from $70.18 \%$ to $77.29 \%$. Even though the existence of a price premium associated with glass packaging may reflect the higher cost of the material (Silayoi and Speece, 2004), the large implicit prices indicate that using glass packaging may also confer an image of a higher quality. The estimated coefficients associated with variables capturing different package size (Medium Pack and Large Pack) are negative, consistent with the expectation that unitary price declines with size; the SF implicit price discounts for both Medium Pack and Large Pack are larger than the OLS ones, to indicate larger discounts at the buyer's price frontier, in particular for the latter, with values as large as $-27.9 \%$. 


\section{Conclusions, limitations and future research.}

In this article, we assess whether manufactures' investments in credence attributes enables them to reach systematically higher price levels. Using the Italian yogurt market as a case study, and a hedonic price model estimated by means of a Stochastic Frontier (SF) estimator we find evidence that manufacturers with a larger portfolio of products carrying credence attributes can systematically reach consumers with a higher willingness to pay. This suggests that introducing credence attributes in a producer's portfolio may lead to successful product differentiation and an increase in reputation, while other attributes seem to lead to lower price premiums once the "buyer's price frontier" is reached. As a result, firms which invest strategically in credence features may benefit in increased reputation and may be able to segment the market more effectively than other firms. Firms that lack the resources to include products carrying health claims in their portfolio, may attempt to reach the "buyer's price frontier" by specializing in the niche of natural or organic products.

In spite of the insights provided in this article, our analysis' value should be contextualized, as our results can be expanded in different directions. First, our analysis focused on one specific case study, the Italian yogurt market, chosen because of its richness in credence product attributes, and because it lends well to the empirical approach used (hedonic price model). The ability of food manufacturers to product differentiate successfully by means of credence attributes should be confirmed by studying other product markets and geographical contexts. Second, our analysis does not explicitly take into account firms' costs related to providing credence attributes; even though our estimates point to the manufacturers' ability to reach higher prices, such higher prices may not result in a higher profitability if the cost of providing credence attributes is prohibitively high. Thus, future research should assessing formally the profitability of these attributes. Third, and last, although we cannot exclude $a$ priori the existence of market asymmetries on both the consumers' and the manufacturers' side, due to our data not containing detailed information on consumers' characteristics, we could only limit our analysis to studying the sources of price dispersion from the manufacturers' standpoint, using what can be seen as a "production frontier" estimation approach. Future research, using richer, more granular data containing both consumers' and manufacturers' characteristics could venture in investigating the existence of two sources of asymmetries, adapting the two-tiered, one-sided error estimator, a la Kumbakhar and Parmeter (2010) to the context of differentiated product markets.

\section{References}

Annunziata, A. and Vecchio, R. (2013). Consumer perception of functional foods: A conjoint analysis with probiotics. Food Quality and Preference 28(1): 348-355.

Ares, G. and Gámbaro, A. (2007). Influence of gender, age and motives underlying food choice on perceived healthiness and willingness to try functional foods. Appetite 49(1): 148158.

Armstrong, M. and Vickers, J. (2001). Competitive price discrimination. Rand Journal of 
Chapter 4

economics 32(4): 579-605.

Barron, J., Taylor, B. and Umbeck, J. (2004). Number of sellers, average prices, and price Dispersion. International Journal of Industrial Organization 22(8): 1041-1066.

Berger, J., Draganska, M. and Simonson, I. (2007). The influence of product variety on brand perception and choice. Marketing Science 26(4): 460-472.

Bimbo, F., Bonanno, A., Nocella, G., Viscecchia, R, Nardone, G., De Vitiis, B., and D. Carlucci (2017). Consumers' acceptance and preferences for nutrition-modified and functional dairy products: A systematic review. Appetite. Advance Access. Available at http://dx.doi.org/10.1016/j.appet.2017.02.031

Bimbo, F., Bonanno, A. and Viscecchia, R. (2016). Do health claims add value? The role of functionality, effectiveness and brand. European Review of Agricultural Economics 43(5): 761-780.

Bonanno, A. (2012). Some like it healthy: demand for functional and conventional yogurts in the Italian market. Agribusiness: an International Journal 28(1): 67-85.

Bonanno, A. (2013). Functional foods as differentiated products: the Italian yogurt market. European Review of Agricultural Economics 40(1): 45-71.

Bonroy, O. and Constantatos, C. (2008). On the use of labels in credence goods markets. Journal of Regulatory Economics 33(3): 237-252.

Bonroy, O. and Constantatos, C. (2015). On the economics of labels: how their introduction affects the functioning of markets and the welfare of all participants. American Journal of Agricultural Economics 97(1): 239-259.

Borenstein, S. and Rose, N. L. (1994). Competition and price dispersion in the U.S. airline industry. Journal of Political Economy 102(4): 653-683.

Brookes, G. (2010). Economic impact assessment of the European Union (EU)'s Nutrition \& Health Claims Regulation on the EU food supplement sector and market. GBC Ltd, UK. Report for the European Health Claims Alliance (EHCA). 57 p. http://www.pgeconomics.co.uk/pdf/Impact-Assessment-health-claims.pdf. Accessed 21 February 2014.

Burdett, K. and Judd, K.L. (1983). Equilibrium price dispersion. Econometrica: Journal of the Econometric Society 51(4): 955-969.

Carlucci, D., Stasi, A., Nardone, G. and Seccia, A. (2013). Explaining price variability in the Italian yogurt market: a hedonic analysis. Agribusiness: an International Journal 29(2): 194206.

Carriazo, F., Ready, R. and Shortle, J. (2013). Using stochastic frontier models to mitigate omitted variable bias in hedonic pricing models: A case study for air quality in Bogotá, Colombia. Ecological Economics 91: 80-88.

Caswell, J. A. and Mojduszka, E. M. (1996). Using informational labeling to influence the 
market for quality in food products. American Journal of Agricultural Economics 78(5): 1248-1253.

Caswell, J. A. and Padberg, D.I. (1992). Toward a more comprehensive theory of food labels. American Journal of Agricultural Economics 74(2): 460-468.

Costanigro, M. and McCluskey, J.J. (2011). Hedonic price analysis in food markets. In The Oxford Handbook of the Economics of Food Consumption and Policy, ed. by J. Roosen, L. J. Lusk, and F. Jason, 153-161. Shogren: Oxford University Press.

Costanigro, M., McCluskey, J.J and Mittelhammer, R.C. (2007). Segmenting the wine market based on price: Hedonic regression when different prices mean different products. Journal of Agricultural Economics 58(3): 454-466.

Costanigro, M., McCluskey, J.J. and Gomans, C. (2010). The Economics of nested names: name specificity, reputations, and price premia. American Journal of Agricultural Economics 92 (5): 1339-1350.

Darby, MR. and Karni, E. (1973). Free competition and the optimal amount of fraud. Journal of Law Economics 16(1): 67-88.

Devine, D. G. and Marion, B. W. (1979). The influence of consumer price information on retail pricing and consumer behavior. American Journal of Agricultural Economics 228-237.

Draganska, M. and Jain, D. (2005). Product-line length as a competitive tool. Journal of Economics \& Management Strategy 14(1): 1-28.

Hovhannisyan, V. and Bozic, M. (2013). A benefit-function approach to studying market power: an application to the U.S. yogurt market. Journal of Agricultural and Resource Economics 38(2): 159-173.

Kalita, J.K., Jagpal, S. and Lehmann, D.R. (2004). Do high prices signal high quality? A theoretical model and empirical results. Journal of Production and Brand Management 13(4): 279-288.

Kamakura, W.A. and Moon, S. (2009). Quality-adjusted price comparison of nonhomogeneous products across internet retailers. International Journal of Research in Marketing 26(3): 189-196.

Kennedy, P. E. (1981). Estimation with correctly interpreted dummy variables in semilogarithmic equations. The American Economic Review 71(4): 801.

Krutulyte, R., Grunert, K.G., Scholderer, J., Lähteenmäki, L., Hagemann, K.S., Elgaard, P., Nielsen, B. and Graverholt, J.P. (2011). Perceived fit of different combinations of carriers and functional ingredients and its effect on purchase intention. Food Quality and Preference 22(1): $11-16$.

Kumbhakar, S.C. and Lovell, C.A.K. (2000). Stochastic frontier analysis. New York: Cambridge University Press.

Kumbhakar, S.C. and Parmeter, C.F. (2009). The effects of bargaining on market outcomes: 
evidence from buyer and seller specific estimates. Journal of Productivity Analysis 31(1): 114.

Kumbhakar, S.C., and Parmeter, C.F. (2010). Estimation of hedonic price functions with incomplete information. Empirical Economics 39(1): 1-25.

Lach, S. (2002). Existence and persistence of price dispersion: an empirical analysis. Review of economics and statistics 84(3): 433-444.

Lancaster, K. J. (1966). A new approach to consumer theory. Journal of political economy 74(2): 132-157.

Lassoued, R. and Hobbs, J. E. (2015). Consumer confidence in credence attributes: The role of brand trust Food Policy 52: 99-107.

Lee, J-D., Park, C.O., Oh, D-H. and Kim, T-Y. (2008). Measuring consumption efficiency with utility theory and stochastic frontier analysis. Applied Economics 40(22): 2961-2968.

Lewis, M. (2008). Price dispersion and competition with differentiated sellers. The Journal of Industrial Economics 56(3): 654-678.

Lodorfos, G. N. and Dennis, J. (2008). Consumers' Intent: in the organic food market. Journal of Food Products Marketing 14(2): 17-38.

McCluskey, J. (2000). A game theoretic approach to organic foods: An analysis of asymmetric information and policy. Agricultural and Resource Economics Review 29(1): 1-9.

Nelson, P. (1970). Information and consumer behavior. Journal of political economy 78(2): 311-329.

Regulation (EC) No 1924/2006 of the European Parliament and of the Council of 20 December 2006 on nutrition and health claims made on foods.

Richards, T. J., Hamilton, S.F. and Allender, W. (2016). Search and price dispersion in online grocery markets. International Journal of Industrial Organization 47: 255-281.

Rosen, S. (1974). Hedonic prices and implicit markets: Product differentiation in pure competition. Journal of Political Economy 82(1): 34-55.

Salop, S. C. and Stiglitz J.E. (1982). The theory of sales: A simple model of equilibrium price dispersion with identical agents. American Economic Review 72(5): 1121-1130.

Salop, S. C. and Stiglitz, J. E. (1977). Bargains and ripoffs: A model of monopolistically competitive price dispersion. The Review of Economic Studies 44(3): 493-510.

Salop, S.C. (1977). The noisy monopolist: imperfect information, price dispersion and price discrimination. The Review of Economic Studies 44(3): 393-406.

Schuldt, J.P. (2013). Does green mean healthy? Nutrition label color affects perceptions of healthfulness. Health Communication 28(8): 814-821.

Schuldt, J.P. and Schwarz, N. (2010). The "organic" path to obesity? Organic claims influence calorie judgments and exercise recommendations. Judgment and Decision Making 
Chapter 4

5(3): $144-150$.

Siegrist, M., Stampfli, N. and Kastenholz, H. (2008). Consumers' willingness to buy functional foods. The influence of carrier, benefit and trust. Appetite 51(3): 526-529.

Silayoi, P. and Speece, M. (2004). Packaging and purchase decisions: A focus group study on the impact of involvement level and time pressure. British Food Journal 106(8): 607-628.

Sorensen, A.T. (2000). Equilibrium price dispersion in retail markets for prescription drugs. Journal of Political Economy 108(4): 833-850.

Sporleder, T. L. and Goldsmith, P. D. (2001). Alternative firm strategies for signaling quality in the food system. Canadian Journal of Agricultural Economics 49(4): 591-604.

Stigler, G. J. (1961). The economics of information. Journal of Political Economy 69(3): 213-225.

Teisl, M. F. and Roe, B. (1998). The economics of labeling: an overview of issues for health and environmental disclosure. Agricultural and Resource Economics Review 27: 140-150.

Verbeke, W. (2005a). Consumer acceptance of functional foods: socio-demographic, cognitive and attitudinal developments. Food Quality and Preference 16(1): 45-57.

Verbeke, W. (2005b). Agriculture and the food industry in the information age. European Review of Agricultural Economics 32(3): 347-368.

Villas-Boas, S. B. (2007). Vertical relationships between manufacturers and retailers: Inference with limited data. The Review of Economic Studies 74(2): 625-652.

Wildenbeest, M. R. (2011). An empirical model of search with vertically differentiated products. The RAND Journal of Economics 42(4): 729-757.

Zhao, Y. (2006). Price dispersion in the grocery market. The Journal of Business 79(3): 11751192 . 
Chapter 4

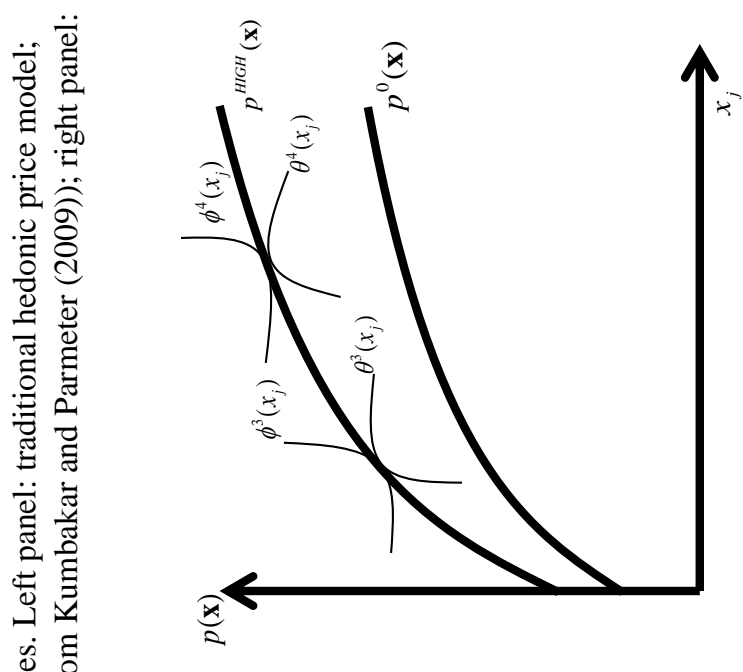

है

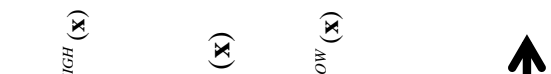

岂 胥

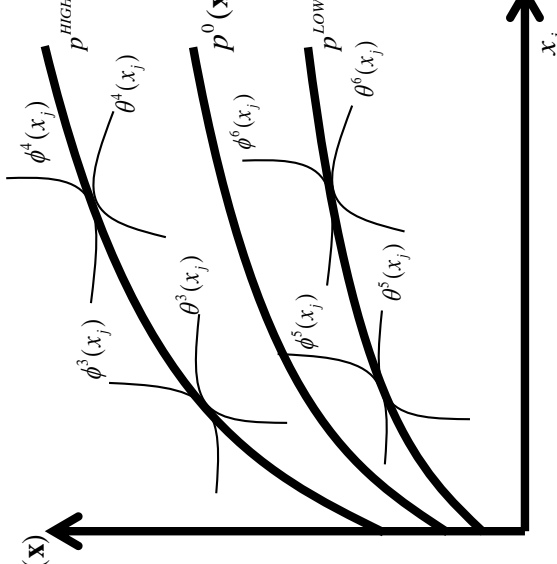

을

苟

गे

흘.

정 들

늘

苛造

¿

类

늘

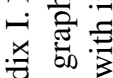

吾高

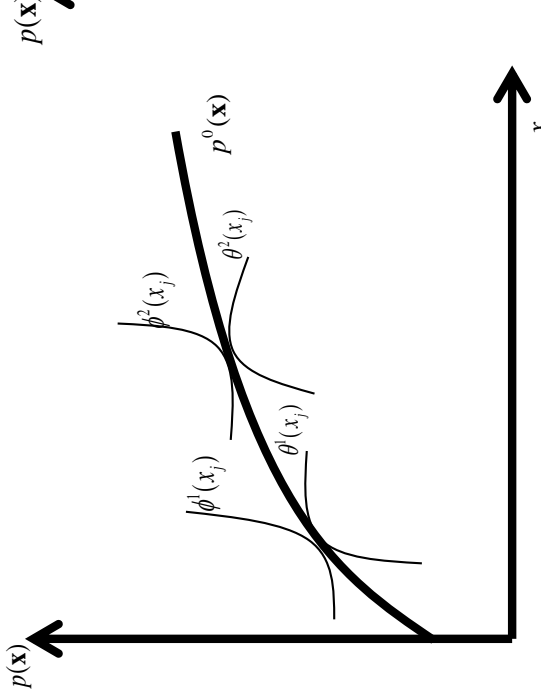


Chapter 4

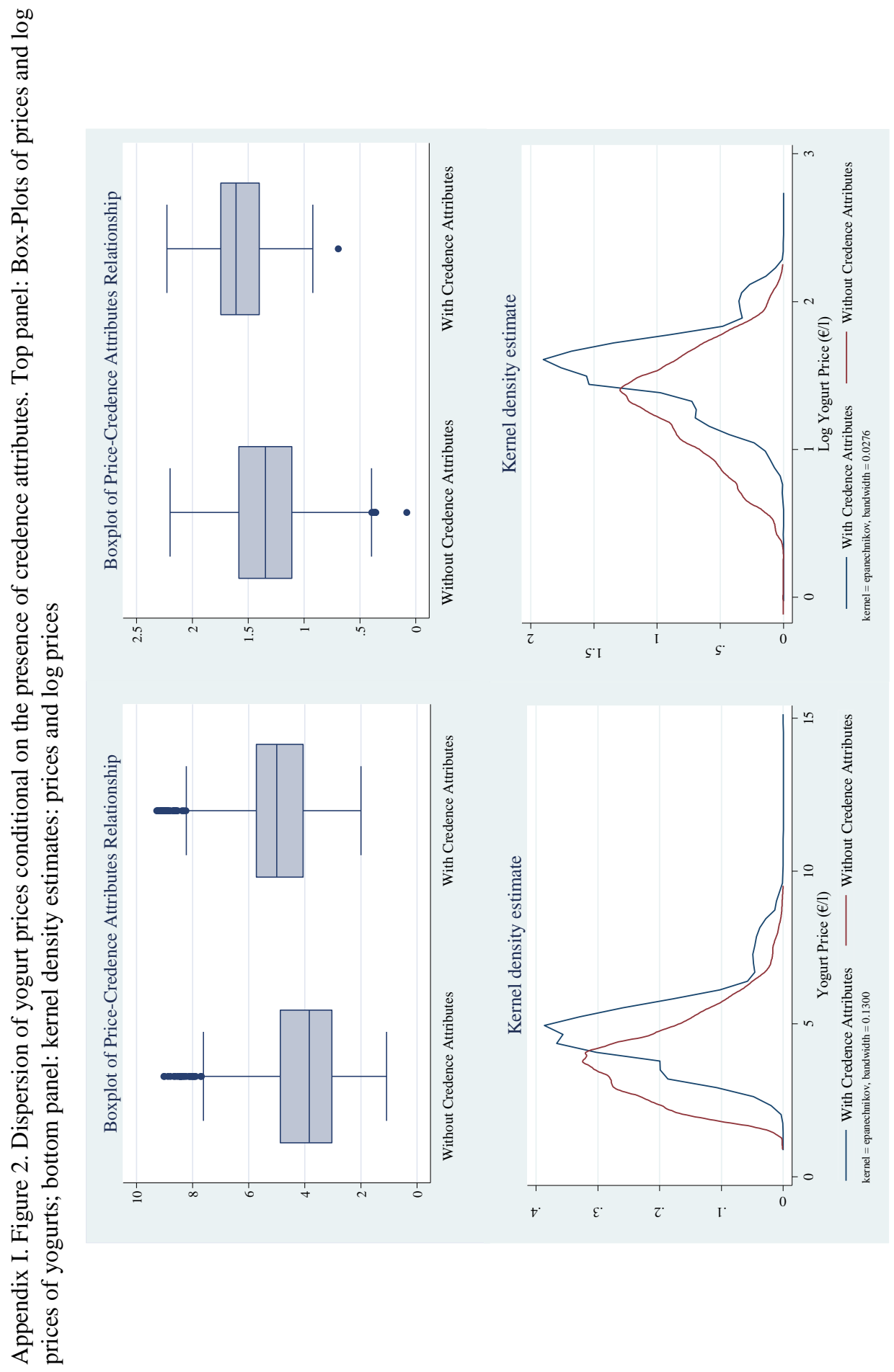




\section{Chapter 5}

Body Image Dissatisfaction and Health-Enhancing Food Choices - An Analysis of Italian Yogurt Consumers.

Francesco Bimbo, Alessandro Bonanno, Hans Van Trijp, Rosaria Viscecchia.

Paper submitted to a peer review journal 


\title{
Body Image Dissatisfaction and Health-Enhancing Food Choices - An Analysis of Italian Yogurt Consumers
}

\begin{abstract}
Body image dissatisfaction, and the negative feelings associated with it, may lead the adoption of unhealthy eating behaviors, to discourage engaging in long term healthy eating habits, and to paying less attention to the quality of food consumed. As a result, body image may affect consumers' decisions of choosing health-enhancing food products, or those with health claims. In this study, we use a sample of Italian yogurt consumers and a virtual-shelf data collection method to study the relationship between respondents' level of body image dissatisfaction and their decision of choosing functional yogurts. We frame our analysis using the self-determination theory, a broad framework used to explain health behaviors, including eating regulation mechanisms. Our results indicate that body image dissatisfaction is negatively associated with the number of yogurt packages with health-enhancing features chosen from the virtual shelf. Further, consistently with self-determination theory, respondents who read the nutrition label and show more knowledge regarding leading functional yogurt brands, purchase a higher number of functional versions from the shelf compared to others, result which is more marked in women respondents. An analysis of predicted functional yogurt purchases conditional on respondents' level of body image dissatisfaction, gender, reading nutrition labels, and leading functional yogurt brands knowledge, suggests potential synergies of these factors in the purchase of health-enhancing products, which warrants additional investigation.
\end{abstract}

Keywords: food choices, self-determination theory, body image dissatisfaction, functional foods.

\section{Introduction}

Body image dissatisfaction encompasses a broad spectrum of negative feelings about a person's own body, such as the sense of mismatch between one's actual body size and the perceived one, or its comparison to the ideal one (Ogden, 2012). These negative feelings can result in concerns about body weight and body shape, leading individuals to engage in compensatory mechanisms (such as extreme diets and unhealthy weight control practices) which increase the likelihood to incur in long-term eating disorders (Thogersen-Ntoumani et al., 2010; Ogden, 2012; Verstuyf, 2012a). Individuals undertake such compensatory mechanisms pursuing the goal of having a pleasant physical appearance, even though such actions have a short-term effect and a negative impact on food choices (Grigeg et al., 1996; Goldfield et al., 2010). Furthermore, social expectations related to having a pleasant body image, such as striving for peers' approval, generate additional negative feelings such as own body shame, exacerbating unhealthy compensatory mechanisms which neither motivate 
individuals to select healthier food options (e.g. vegetables), nor promote long-term healthy eating behaviors (Verstuyf et al., 2012a; 2012b; Markey et al., 2016).

Using self-determination theory (SDT), Verstuyf et al., (2012b) indicate that individuals who modify their food habits mainly because of a sense of pressure to conform to others' demands, their approval, or to avoid criticisms, are more likely to adopt unhealthy eating strategies to ameliorate their body image, and are also more exposed to the risk of eating disorders. SDT was developed by Deci \& Ryan (1985) and focuses on the degree to which human behavior is self-determined, or the extent to which individuals engage in actions with a sense of personal choice (Deci \& Ryan, 1985). Specifically, human behavior is expected to arise from motivational foundations that range from intrinsic (autonomous) to extrinsic (controlled). Autonomously regulated behavior promotes more adaptive psychological and behavioral functioning, while controlled behavior is associated with lower psychological wellbeing and less effective behaviors (Deci \& Ryan, 2002). Thus, SDT predicts that individuals willing to reach a goal that is not personally endorsed (e.g. peer pressure, feelings of guilt) may lack self-determined actions and are prone to discouragement after negative experiences or failures, and are less persistent in reaching the desired outcome (Deci \& Ryan, 1987). Instead, people who are engaged in activities that reflect their own interests, their own values, mostly act in a self-determined way and are more likely to achieve their goals (Deci \& Ryan, 2002).

Verstuyf et al. (2012b) suggested that individuals who modify their own eating habits to reach a goal, while valuing the importance of staying in good health, are more likely to engage in healthier eating strategies to mitigate their level of body image dissatisfaction, compare to others. Previous research exists evaluating the outcomes, motivations and goals behind adopting healthy (or unhealthy) eating habits according to SDT. Pelletier et al. (2004a; 2007) found that individuals who engage in controlled forms of eating habits to maximize weight loss pay little attention to the variety and quality of the food they ingest, as they are more interested in how much they eat rather than what they eat ${ }^{1}$. Also, from a SDT perspective, women are more likely than men to engage in autonomous actions to protect their own health against the adverse effects of societal demand to have a thin body ${ }^{2}$ (Pelletier et al., 2004b; Pelletier \& Dion, 2007; Mask et al., 2010). Individuals who act in more self-determined ways were found to be less likely to engage in disordered eating behaviors (e.g., bulimic symptoms) and more likely to engage in healthy ones (e.g., eating higher amounts of vegetables) (Pelletier et al., 2004a).

The aim of this work is to explore empirically the relationship between consumers' declared level of body dissatisfaction and their food choices. In assessing this relationship, we will also test whether individuals who act in a more self-determined way (e.g. using nutritional labels,

\footnotetext{
${ }^{1}$ Additionally, long fasting and unhealthy weight control behaviors increase stress level and can often lead to overeating phases which rebalance calorie loss and nullify efforts to reach the ideal body shape, in many cases bringing an excess of calories to the body with a consequent weight increase (Johnson \& Wardle, 2005) and higher risks of diet-related diseases.

2 Although both men and women can experience body image dissatisfaction, women generally face lower levels of body satisfaction compared to men as they are subject to a higher societal pressure to achieve a thin body (e.g., thin-ideal media images) (Wardle, Haase, \& Steptoe, 2006; Algars et al., 2009). However, women have usually higher nutritional knowledge than men, thus being more likely to select healthy food options (Drichoutis et al., 2006; Miller et al., 2015).
} 
or having acquired prior knowledge) when making food choices, purchase a higher number of health-enhancing food options. In order to achieve these goals, we collected data from a sample of Italian yogurt consumers using a virtual supermarket shelf containing yogurts with and without health claims, asking them to select their preferred products, and then collected information on their level of body image dissatisfaction, functional product knowledge (with respect of yogurt leading brands), their use of nutrition labels when shopping for food and, as well as other socio-demographic characteristics.

We used a virtual shelf as a data collection method as it exposes respondents to an assortment of food products resembling those available in supermarkets, simulating real-life shopping behavior more closely than survey-based methods. As a result, data collected through virtual supermarket shelves are highly correlated with actual sales data (Burke, 1996). The participants of our study were asked to purchase yogurts from the virtual shelf as if they were in the supermarket where they usually purchase food. Then, the virtual shelf software recorded the number of yogurt packages each consumer purchased carrying health-claims or health-enhancing features. The yogurt category was in fact chosen as a case study because of its high level of differentiation in term of health-enhancing features (Siegrist et al., 2008; Sirò, 2008; Ares et al., 2010; Bonanno, 2012). Thus, we use the number of product options purchased by respondents belonging to these "healthier" product subcategories, as a means to measure respondents' healthy eating behavior and their willingness to invest in health through the diet.

The remainder of the paper is structured as follows. Section 2 describes the data collection process and the advantages of using a virtual shelf methodology, followed by a description of the empirical framework and estimation. Section 3 and 4 present the empirical results of the study and their discussion, respectively. Section 5 concludes with final remarks and a discussion of possible avenues for future research.

\section{Materials and methods}

Data collection was performed by means of a virtual shelf. This data collection method increases the realism and it simulates more closely the complexity of a "real" choice environment, compared to survey-based choice experiments which usually employ a limited number of pictorial stimuli (Burke, 1992; 1996; van Herpen et al., 2016). Also, methods based on virtual reality allow to capture consumers' variety seeking behavior as participants are free to select and evaluate one or more products from the shelf before making their choices (Simonson, 1990). Additionally, research has shown that simulated sales data collected with this method are highly correlated with actual ones (Burke, 1992; 1996; Campo, 1999), increasing the external validity of the choice experiment performed. The use of virtual shelves represents a novel approach for data collection in consumer studies on functional products; to the best of our knowledge, the majority of consumer behavior studies with respect of health-enhancing food products have used "fictional" product concepts (Burke, 
1996; van Kleff et al., 2005; Ares \& Gámbaro, 2007; Krutulyte et al., 2008; 2011; Ares et al., 2009; Hailu et al., 2009; Ares and Deliza, 2010; Annunziata \& Vecchio, 2013).

\subsection{Study participants}

Two hundred and fifty individuals were recruited from a sample of Italian yogurt consumers to participate in our study by IPSOS, a marketing consulting company. The selected participants were yogurt shoppers between 18 and 60 years of age, equally distributed across gender and resided in the cites of Milan, Rome, and Bari. We selected respondents from the metropolitan areas of Milan, Rome, and Bari as they are located, respectively, in the North, Center and South of Italy, and could allow us to capture regional differences in consumer behavior. The individuals recruited could access the server where the virtual yogurt shelf was uploaded through a link received via email. After having reached the virtual shelf, participants were asked to shop for yogurts to simulate in-store purchases (purchase simulation phase). At the end of the purchase simulation phase, the virtual shelf's software collected the number of functional yogurt packages "virtually" purchased by the participants, and then prompted the respondents to participate to an online questionnaire, in order to collect additional information (level of body image dissatisfaction, socio-demographic characteristics, etc.)

\subsection{Virtual shelf design, purchase simulation process and questionnaire description}

\section{Virtual shelf design and features}

The virtual shelf employed in our analysis was designed based upon actual supermarket yogurt shelves from stores located in the same cities where the participants reside (Milan, Rome, and Bari) and belonging to the leading Italian food retail chain (Associazione Nazionale Cooperative di Consumatori - COOP). The yogurt shelf layout of one store in each city was reproduced in a planogram ${ }^{3}$, including the information regarding the packages of each product that was available on the shelf. The information collected in the planogram were: each yogurt's complete name (as in theit label), its European Article Number (EAN) ${ }^{4}$ code, the position of the product on the shelf, the amount of shelf space allocated to each product (facing), the type of yogurt (Greek, for kids, from soy milk, flavor and functional properties), its package size, and its price.

Yogurts found in at least two out of the three planograms were included in the virtual shelf. The final version of the virtual shelf contained 92 national brands and 20 private labels for a total of 112 products. Each product included in the shelf was purchased from a supermarket, photographed in high resolution (front, back, sides, top and bottom) and its pictures used to build the virtual shelf. Each product's position on the virtual shelf replicated that of the inspected supermarkets. A product's price and its number of facing in the virtual shelf (that is,

\footnotetext{
${ }^{3}$ The planogram is a visual diagram showing where specific products should be located on a retail shelf also reporting product information. ${ }^{4}$ The EAN code is a 13-digit code used in Europe at the retail level to identify products carrying a barcode to be read by scanner cash registers.
} 
number of items visible on the shelf), were obtained from the average price and the number of facings recorded in the supermarkets surveyed where the product was available.

\section{Consumers purchase simulation process and questionnaire description}

The recruited participants were asked to select any product they were interested into from the virtual shelf. Each product selected by means of the computer's cursor, automatically moved to the foreground so that respondents could rotate the image and examine all of the product's faces (see Figure 1). Then, participants decided whether to put the product selected in their shopping basket or not, with the option to remove products from their basket at any time before checking out.

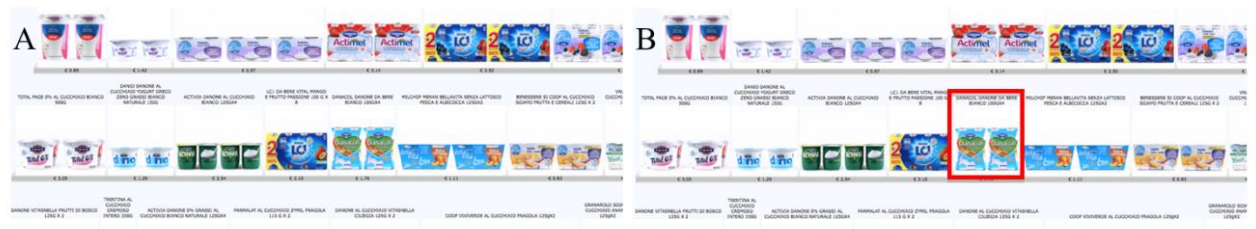

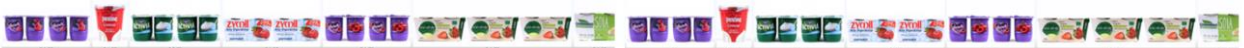

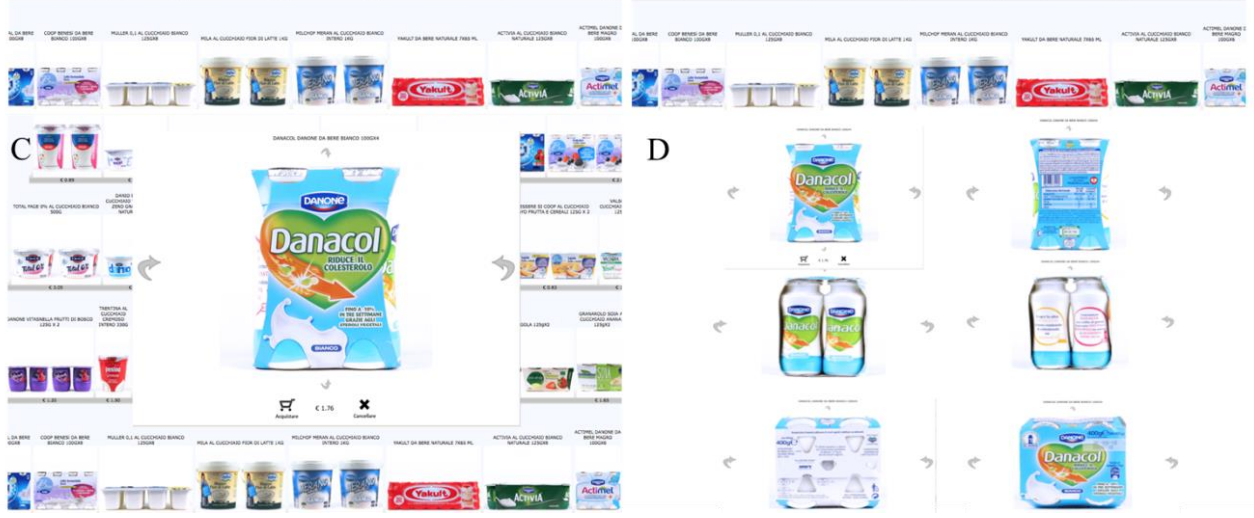

Figure 1. Virtual shelf and product selection process. Panel A, top right: snapshot of a portion of the virtual shelf. Panel B, top left: simulated selection of a product from the shelf. Panel C, bottom left: the selected product is moved to the foreground. Panel $\mathrm{D}$, bottom right: product is rotated on all of its faces.

During the purchase process, the software kept track of all products purchased by each participant, and whether the products still in the basket at checkout were functional or not. After completing the shopping simulation, each consumer filled out a survey.

The survey collected information on the respondents' level body of dissatisfaction by asking them to select the best fitting statement regarding their assessment of their body image, as referred to the experience of watching themselves in the mirror: "I like my body", "I accept my body", "I do not accept my body", and "I do not like my body, I envy other people's". 
The survey recorded also information about participants' level of familiarity with the leading brands of functional yogurts, as brand recognition plays an important role in the purchase of functional alternatives (Bimbo et al., 2016; 2017). The respondents' familiarity with the leading brands of functional yogurts was measured on a scale 0-10. This scale was constructed by asking respondents to match ten statements, each referring to a feature of one of the leading functional yogurt brands (e.g. the functional enrichment, or the health claim) with the product they belong to. Every correct match resulted in 1 point on the consumers' familiarity scale. A score of 0 was assigned to respondents with all incorrect matches (that is, "fully unfamiliar" respondents), while a score of 10 to those whose matches were all correct ("highly familiar" respondents). A separate question collected information on the respondents' frequency of use of the nutrition labels when shopping for food (options "Always", "Sometimes", "Never"). The information on pre-existing functional yogurt knowledge and the usage of nutritional labels was collected to obtain metrics of the respondents' propensity to undertake autonomous actions in food choices. Lastly, the survey recorded participants' socio-demographic characteristics such as, age, gender, household size, education level, current job, place of residence, direct or indirect (through a family member or relative) exposition to chronic diseases, frequency of practicing physical activity, and primary type of outlet used for food shopping (hypermarkets/supermarkets, grocery stores, or proximity stores).

\subsection{Data description}

Of the selected 250 participants, 21 of them did not complete the virtual shopping experience and/or the survey, for a total of 229 usable observations. The average number of products retained in the shopping basket at check out (i.e. purchased products) was 3.6, with an average number of functional alternatives retained of 0.917 . Slightly less than the $50 \%$ of participants retained in their shopping basket at least one package of functional yogurts.

With respect to the level of body dissatisfaction, the majority of respondents $(66.4 \%)$ declares to accept her or his own body, approximately one out of five $(20.9 \%)$ to like it, while the remaining part of the sample $(12.7 \%)$ declares not to like their own body or to envy those of others. Respondents in our sample show an "average" level of familiarity with the leading brands of functional yogurt, as the mean of the 0-10 scale variable capturing individual's familiarity for these products was 5.1. Slightly more than two thirds of our respondents $(68.6 \%)$ declared to read nutrition food label ("Always", or "Sometimes") when purchasing food.

With respect to the demographic variables, $51.1 \%$ of the respondents is female and the average age is approximately 41 years. With respect to education, $54.1 \%$ of the respondents completed high school, $38.0 \%$ has a university degree, while the rest $(7.9 \%)$ completed middle school or had a lower level of education. The $59.4 \%$ of the respondents declared to be a full time worker, the $20.5 \%$ part time, while the remaining $20 \%$ declared to be unemployed. The majority of the respondents $(86.3 \%)$ belongs to a households with 2 to 4 components, for 
a 3.15 average household size. The $40.6 \%$ of respondents declared to have been directly or indirectly (through a family member or a relative) exposed to chronic diseases. Almost half $(48.5 \%)$ of the respondents practice sport regularly or occasionally. Lastly, the majority of the respondents usually shop for food in grocery stores $(58.5 \%)$, about a third $(32.8 \%)$ in a supermarket/hypermarket, and the rest $(8.7 \%)$ in proximity stores. Summary statistics are presented in Table 1.

Table 1. Variable description and summary statistics (229 observations).

\begin{tabular}{|c|c|c|}
\hline Variables & Description & Mean \\
\hline \multicolumn{3}{|c|}{ Dependent variable } \\
\hline$T P F Y$ & $\begin{array}{l}\text { Range } 0-7=\text { Total packages of functional yogurts purchased from } \\
\text { the Virtual Shelf. }\end{array}$ & $\begin{array}{c}0.917 \\
(1.3169)\end{array}$ \\
\hline \multicolumn{3}{|c|}{ Looking at the mirror as you evaluate your body image ( $B O D Y)$ : } \\
\hline Like & 1= I like my body; & 0.209 \\
\hline Accept & $1=\mathrm{I}$ accept my body; & 0.664 \\
\hline Do_Not_Like & 1= I do not like my body; & 0.118 \\
\hline Envy_others & 1= I do not like my body and I envy other peoples'. & 0.009 \\
\hline
\end{tabular}

Respondent familiarity scale with leading functional yogurt brands $(F K)$ :

FK Range 0-10; $0=$ fully unfamiliar; $10=$ highly familiar.

How frequently respondent reads nutrition food label before purchasing food products $(N L)$ :

$N L$

$1=$ Always or Sometimes.

0.686

Respondent socio-economics characteristics $(S E C)$ :

\begin{tabular}{|c|c|c|}
\hline Gender & $1=$ Female; & 0.511 \\
\hline Age & Range $18-60=$ Respondent's age; & 41.035 \\
\hline Univ & 1= Highest level of education achieved: bachelor degree or higher; & 0.380 \\
\hline High & 1= Highest level of education achieved: High school degree; & 0.541 \\
\hline LessHigh & 1= Highest level of education achieved: Less than High school; & 0.079 \\
\hline FullTime & 1= Employment Status: Full-time worker; & 0.594 \\
\hline ParTime & 1= Employment Status: Part-time worker; & 0.205 \\
\hline Unemp & 1= Employment Status: Unemployed; & 0.200 \\
\hline HH_size & Range 1-8= Household size; & 3.148 \\
\hline$H H \_s i z e^{\wedge} 2$ & Range 1-64= Household size squared; & 11.140 \\
\hline Chronic & $\begin{array}{l}1=\text { if respondent has direct, or indirect, experience with chronic. } \\
\text { diseases (arthritis, diabetes, osteoporosis, heart disease, tumours). }\end{array}$ & 0.406 \\
\hline$P A$ & $1=$ Yes. & 0.485 \\
\hline \multicolumn{3}{|c|}{ tore where respondents regularly shops for food (STORE): } \\
\hline Hyp_Sup & 1= Supermarket/hypermarket; & 0.328 \\
\hline Gro & 1= Grocery Store; & 0.585 \\
\hline Prox & 1= Proximity store. & 0.087 \\
\hline
\end{tabular}

Place where respondents live $(C I T Y)$ :

$\begin{array}{lll}\text { Milan } & \text { 1= Milan; } & 0.345 \\ \text { Rome } & \text { 1= Rome; } & 0.314 \\ \text { Bari } & \text { 1= Bari. } & 0.341\end{array}$

${ }^{\mathrm{a}}$ For all binary variables the standard deviation is omitted as the mean, reported in table, represents the share. 
One of the advantages of using data collected through virtual shelf is that they provide a good approximation of real market data, as they can be highly correlated to sales data (Burke, 1996). Figure 2 compares the aggregate share of respondents' choices at the brand level collected using the virtual shelf (red dot line) with the share of volume sales at the brand level obtained from sales data provided by SymphonyIRI ${ }^{5}$, courtesy of the University of Foggia, Italy (blue line). ${ }^{6}$ The high correlation value is in line with Burke's (1996) findings, lending validity to our assumption that our virtual shelf data can give us a good approximation of actual consumer behavior in the marketplace.

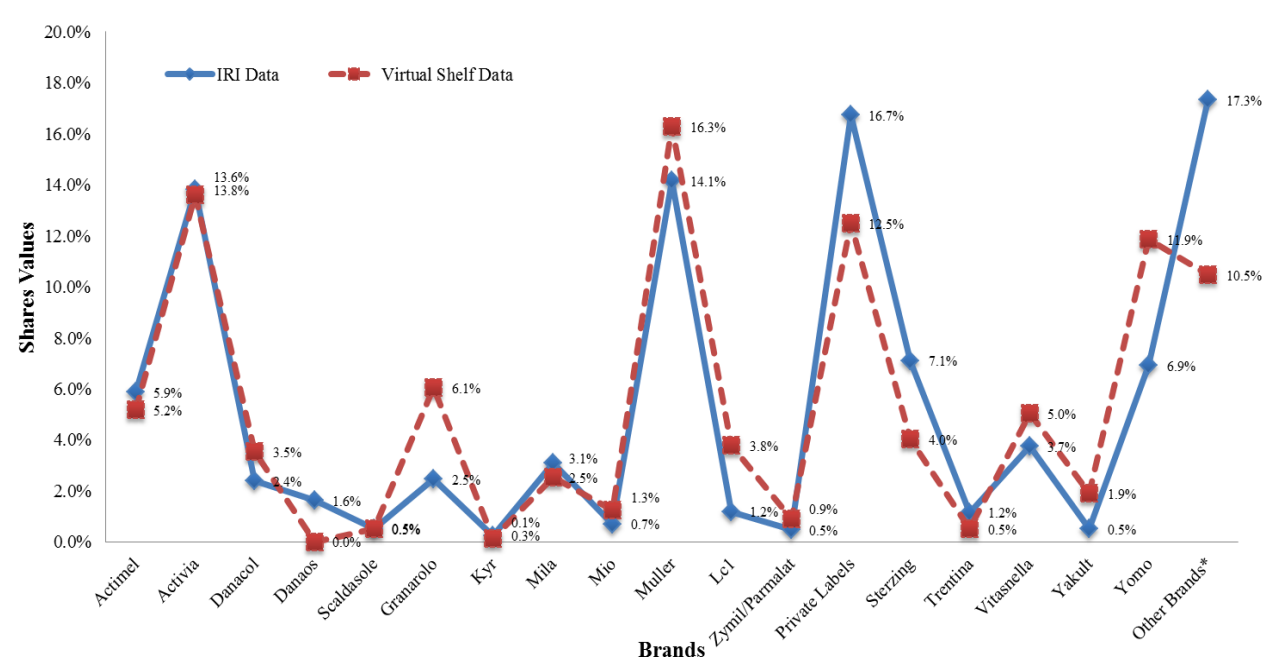

Figure 2. Comparison between IRI share volume sales data (2010-2012) and shares of preferences from virtual shelf method aggregated at brand level.

\subsection{The Model and Data analysis}

To explore the relationships between the number of functional yogurt packages purchased by the respondents (i.e. kept in their virtual shopping basket) and their level of body satisfaction, we posit a simple empirical relationship where the total number of functional yogurt packages purchased from the virtual shelf by respondent $i\left(T P F Y_{i}\right)$ is function of the self-declared level of body satisfaction $\left(B O D Y_{i}\right)$ and a series of covariates (control variables) capturing respondent's familiarity with the functional leading branded yogurt $\left(F K_{i}\right)$ and the frequency of nutrition label use when choosing food $\left(N L_{i}\right)$; individuals' socio-economic characteristics $\left(S E C_{i}\right.$; i.e. age, gender, education household size, etc.) and whether they practice physical

\footnotetext{
${ }^{5}$ The SymphonyIRI database contains information about product sales collected monthly at the point-of-sale and then aggregated at the regional level. Such data come from the whole Italian market encompassing a 25-month period between November 29, 2010, and December 31,2012 . The data are recorded at the EAN level.

6 "Other brands" category encompasses regional/local yogurt brands, soy and kids yogurt brands as well as Greek ones.
} 
activity $\left(P A_{i}\right)$; the type of store where respondent $i$ shops food (STORE $;$; i.e. supermarket/hypermarket, grocery store or proximity store); and last, the place where respondents live $\left(C I T Y_{i}\right)$. Such empirical specification results in the following equation:

$$
\begin{gathered}
\text { TPFY }_{i}=\alpha+\sum_{b=1}^{B} \beta_{B O D Y_{b}} B O D Y_{i b}+\sum_{f=1}^{F} \beta_{F K_{f}} F K_{i f}+\sum_{n=1}^{N} \beta_{N L_{n}} N L_{i n}+\sum_{s=1}^{S} \beta_{S E C_{s}} S E C_{i s}+\sum_{p=1}^{P} \beta_{P A_{p}} P A_{i p}+ \\
\sum_{s=1}^{S} \beta_{S T O_{s}} S T O R E_{i s}+\sum_{c=1}^{C} \beta_{C I T Y_{c}} \operatorname{CITY}_{i c}+\varepsilon_{i}
\end{gathered}
$$

Where the $\beta_{\mathrm{s}}$ are the parameters to be estimated, $\varepsilon_{i}$ is an error term and the explanatory variables are discussed above and further explained in Table 1.

As our dependent variable is non-negative and discrete, a count data model is the natural candidate for the estimation. The benchmark estimation tool for count data analysis is the Poisson regression model, which assumes that the dependent variable (in our case $T P F Y_{i}$ ) is draw from a Poisson distribution with parameter $\lambda_{i}$. The relationship between the dependent variable $Y$ and the regressors' vector $\mathbf{x}_{i}$ is represented by the following relationship: $\operatorname{Pr}\left\{Y=y_{i} \mid \mathbf{x}_{i}\right\}=\frac{e^{-\lambda_{i}} \lambda^{y_{i}}}{y_{i} !}$, with $\ln \lambda_{i}=\mathbf{x}_{i}^{\prime} \beta_{i}$ and $y_{i}=1,2,3 \ldots$ Thus, the conditional expectation of the dependent variable given the regressors is $E\left[y_{i} \mid \mathbf{x}_{i}\right]=\operatorname{Var}\left[y_{i} \mid \mathbf{x}_{i}\right]=\lambda_{i}=e^{\mathbf{x}_{i}^{\prime} \beta_{i}}$ which assumes equality of conditional mean and variance (equi-dispersion, or "Poisson assumption"). However, if the equi-dispersion assumption is violated, over-dispersion occurs, resulting in inflated $t$-statistics and deflated $p$-values (Cameron and Trivedi, 1998).

In the case of over-dispersion, the Negative Binomial (henceforth NB) is the most common alternative to the Poisson model, where: $\ln \lambda_{i}=\mathbf{x}_{i}^{\prime} \beta_{i}+v_{i}$ with $e^{v_{i}}$ following a Gamma distribution with mean one and variance $\alpha$. Thus, the contribution to the likelihood of the $i^{\text {th }}$ observation of a Poisson-distributed random variable $Y$ is: $\operatorname{Pr}\left\{Y=y_{i} \mid \mathbf{x}_{i}\right\}=\frac{\Gamma\left(\theta+y_{i}\right)}{\Gamma(\theta) y_{i} !} v_{i}\left(1-v_{i}\right)^{y_{i}}, \quad$ with $\quad v_{i}=\theta /\left(\theta+\lambda_{i}\right), \theta=1 / \alpha$ and $\Gamma(\bullet)$ being $\quad$ the

Gamma function. Thus, the NB regression model has the additional over-dispersion parameter $\alpha$ to be estimated, which accounts for differences in magnitude between conditional mean and variance functions: $\operatorname{Var}\left[y_{i} \mid \mathbf{x}_{i}\right]=E\left[y_{i} \mid \mathbf{x}_{i}\right]\left(1+\alpha E\left[y_{i} \mid \mathbf{x}_{i}\right]\right)$. A positive (and statistically different than zero) estimated $\alpha$ indicates the presence of unobserved heterogeneity in the data leading to rejecting the Poisson model in favor of the NB. Using the test for over-dispersion proposed by Cameron and Trivedi (1990) $;^{7}$ we rejected the use of standard Poisson regression model in favor of NB regression.

\footnotetext{
${ }^{7}$ As proposed by Cameron and Trivedi (1990), the test's null hypothesis is $\mathrm{H}_{0}$ : $\alpha=0$, or alternatively $\mathrm{H}_{0}$ : $\mathrm{E}(\mathrm{Y})=\mathrm{Var}(\mathrm{Y})$. In our case, we reject $\mathrm{H}_{0}$ detecting the presence of over-dispersion in our data (for an estimated $\alpha$ of 1.692, and a $p$-value of 0.001 ), rejecting the use of a Poisson regression model in favour of the NB.
} 
However, over-dispersion may also due to the presence of an excessive number of zeros. If this is the case, a zero-inflated NB (ZINB) model would be more appropriate. We used Vuong test statistic (Vuong, 1989) ${ }^{8}$ to test for the presence of "excess zeros" in our data; as the results of the Vuong test rejected the use of ZINB regression in favor of NB, ${ }^{9}$ the NB was the model selected for our estimation. STATA version 14.0 was used for testing and for the estimation.

\section{Results and discussion}

The estimated NB coefficients of equation (1) are presented in Table 2, second column, along with their standard errors, third column. The first thing to be noted is that the $\alpha$ coefficient is positive and statistically significant, indicating the over-dispesion of the dependent variable, and justifying the use of a NB model. The Wald-test statistic indicates that all the estimated coefficients are jointly statistically significant in explaining the number of functional yogurt packages purchased by respondents from the virtual shelf.

The results in table 2 show a negative association between the respondents' declared level of dissatisfaction with their own-body image and the number of functional yogurts retained at check out. Such relationship becomes more negative as the feeling of dissatisfaction increases. In fact, respondents who declared to accept their own body (Accept) purchased a lower number of functional packages compare to those declaring to like it. The magnitude of the negative relationship is larger for respondents who declared not to like their own body (Do_Not_Like) and even larger for those declaring not to like their own body and to envy others' (Envy_others).

Respondent's familiarity with the leading functional yogurt brands is positively associated with respondents purchasing more health-enhancing yogurt options, indicating that respondents purchased more functional packages at higher values of the familiarity scale $(F K)$. Also, respondents using nutrition label "Always", or "Sometimes" when shopping for food $(N L)$ bought more packages of functional yogurts compared to non-label readers.

On average, female yogurt shoppers (Gender) are more likely to purchases more packages of functional food options compared to man. Age does not seem to be associated with retaining more packages of functional yogurts in the shopping cart, as the estimated coefficient for Age is not statistically significant. Further, as expected, higher the level of education is associated with a higher number of functional yogurt packages "virtually" purchased: respondents with high school diploma (High) and university degree (Univ) retain in their food basket more functional yogurt versions than those with less than high school education level (LessHigh).

Respondents with a full time job (FullTime) purchased more functional packages compared to those declaring to be unemployed (Unemp) as well as those living in large household size.

\footnotetext{
${ }^{8}$ The value of the z-statistic was equal to 0.42 with a corresponding $p$-value of 0.3375 , indicating that the zeros in our sample are sufficiently explained by the explanatory variables when a NB regression is used, rejecting the use of a ZINB.

${ }^{9}$ The NB model predicts the outcomes with a $95.6 \%$ of accuracy, and it does not under-predict the zero outcomes in the data, providing additional supporting the decision of using a NB regression instead of a ZINB (Long and Freese, 2006).
} 
Surprisingly, estimates show no statistically significant relationship between respondent's direct or indirect exposition to chronic diseases (Chronic) and the number of functional yogurts packages purchased from the shelf. Lastly, practicing physical activity (PA) is associated with purchasing a lower number of functional yogurts from the virtual shelf. Instead, respondents increase the consumption of health-enhancing options when shop for food in supermarkets or hypermarkets (Hyp_Sup).

Table 2. Estimated coefficients of equation (1): Negative Binomial regression.

\begin{tabular}{lrrl}
\hline Variable & $\beta$ & St. error & sign \\
\hline Self-declared level of body satisfaction $(B O D Y):$ & & \\
Accept & -0.493 & 0.1963 & $* *$ \\
Do_Not_Like & -1.580 & 0.3807 & $* * *$ \\
Envy_others & -1.643 & 0.6106 & $* * *$
\end{tabular}

Respondent familiarity with leading functional yogurt brands $(F K)$ :
FK
0.112
$0.0340 * * *$

How frequently respondent reads nutrition food label before purchasing food products $(N L)$ :

$\begin{array}{lll}N L & 0.436 & 0.2080\end{array}$

Respondent socio-economics characteristics (SEC):

$\begin{array}{lccc}\text { Gender } & 0.424 & 0.1766 & * * \\ \text { Age } & 0.009 & 0.0080 & \\ \text { Univ } & 0.965 & 0.4638 & * * \\ \text { High } & 1.012 & 0.4325 & * * \\ \text { FullTime } & 0.409 & 0.2488 & * \\ \text { ParTime } & 0.204 & 0.2790 & \\ \text { HH_size } & 0.449 & 0.2697 & * \\ \text { HH_size } 2 & -0.064 & 0.0351 & * \\ \text { Chronic } & -0.007 & 0.1748 & \end{array}$

Respondent habit of practicing physical activity regularly during the week $(P A)$ :
$P A$
$-0.331$
$0.1707 *$

Store where respondents shops foods (STORE):

$\begin{array}{lll}\text { Hyp_Sup } & 0.897 & 0.3800 \\ \text { Gro } & * * \\ \text { Place } & 0.622 & 0.3847\end{array}$

Place where respondents live (CITY):

\begin{tabular}{lrrr} 
Milan & 0.254 & 0.1986 & \\
Bari & 0.327 & 0.2128 & \\
Constant & -3.902 & 0.7938 & $* * *$ \\
\hline $\ln \alpha$ & -1.099 & 0.3972 & $* * *$ \\
$\alpha$ & 0.332 & 0.1322 & $* * *$ \\
\hline Wald test $\chi^{2}(\mathrm{df})$ & $70.21(19)$ & & $* * *$ \\
McFadden's adjusted $\mathrm{R}^{2}$ & 0.1077 & & \\
\hline
\end{tabular}

Note: $* * *$ and $* * *$ indicate 10,5 , and 1 per cent significance levels, respectively.

Next, we estimated the predicted number of purchased yogurt packages with healthenhancing features conditionally on the reported level of body image dissatisfaction, gender 
Chapter 5

and whether respondents use nutritional food label "Always", or "Sometimes" when shopping for food, to assess whether respondents' behave in a manner consistent with SDT.

The average predicted number of functional yogurt packages purchased by different respondent sub-samples are presented in Table 3.

Table 3. Average predicted number of functional yogurt packages purchased conditional on body image dissatisfaction, gender, and reading nutritional labels.

\begin{tabular}{|c|c|c|c|c|c|}
\hline \multirow[b]{2}{*}{$\begin{array}{l}\text { Body-Image } \\
\text { Satisfaction }\end{array}$} & \multirow[b]{2}{*}{ Samples } & \multirow[b]{2}{*}{ Full Sample } & \multicolumn{2}{|c|}{ Reading nutrition food labels } & \multirow[b]{2}{*}{$\begin{array}{c}p \text {-val } \\
\text { equality test }\end{array}$} \\
\hline & & & $\begin{array}{c}\text { Not Readers } \\
\text { (Label=0) }\end{array}$ & $\begin{array}{c}\text { Readers } \\
(\text { Label=1) }\end{array}$ & \\
\hline \multirow[t]{4}{*}{ Like } & Full Sample & $\begin{array}{l}1.166 * * * \\
(0.1909)\end{array}$ & $\begin{array}{c}0.716 * * * \\
(0.1676)\end{array}$ & $\begin{array}{c}1.458 * * * \\
(0.2382)\end{array}$ & 0.0003 \\
\hline & Male & $\begin{array}{c}0.927 * * * \\
(0.1793)\end{array}$ & $\begin{array}{c}0.727 * * * \\
(0.1763)\end{array}$ & $\begin{array}{l}1.04 * * * \\
(0.2138)\end{array}$ & 0.1359 \\
\hline & Female & $\begin{array}{l}1.451 * * * \\
(0.2497)\end{array}$ & $\begin{array}{c}0.776 * * * \\
(0.2016)\end{array}$ & $\begin{array}{c}1.684 * * * \\
(0.2861)\end{array}$ & 0.0006 \\
\hline & $p$-val equality test & 0.0087 & 0.7846 & 0.0188 & \\
\hline \multirow[t]{4}{*}{ Accept } & Full sample & $\begin{array}{c}0.712 * * * \\
(0.0781)\end{array}$ & $\begin{array}{c}0.437 * * * \\
(0.0848)\end{array}$ & $\begin{array}{c}0.890 * * * \\
(0.1015)\end{array}$ & 0.0001 \\
\hline & Male & $\begin{array}{c}0.566 * * * \\
(0.0768)\end{array}$ & $\begin{array}{c}0.411 * * * \\
(0.0798)\end{array}$ & $\begin{array}{c}0.717 * * * \\
(0.1038)\end{array}$ & 0.0052 \\
\hline & Female & $\begin{array}{c}0.886 * * * \\
(0.1217)\end{array}$ & $\begin{array}{c}0.537 * * * \\
(0.1339)\end{array}$ & $\begin{array}{c}1.113 * * * \\
(0.1513)\end{array}$ & 0.0003 \\
\hline & $p$-val equality test & 0.0096 & 0.2365 & 0.0117 & \\
\hline \multirow[t]{4}{*}{ Do_Not_Like } & Full sample & $\begin{array}{c}0.240 * * * \\
(0.0812)\end{array}$ & $\begin{array}{c}0.147 * * * \\
(0.0550)\end{array}$ & $\begin{array}{c}0.300 * * * \\
(0.1021)\end{array}$ & 0.0153 \\
\hline & Male & $\begin{array}{c}0.191 * * * \\
(0.0669)\end{array}$ & $\begin{array}{l}0.100 * * \\
(0.0402)\end{array}$ & $\begin{array}{c}0.226^{* * * *} \\
(0.0806)\end{array}$ & 0.0239 \\
\hline & Female & $\begin{array}{c}0.299 * * * \\
(0.1032)\end{array}$ & $\begin{array}{c}0.1087 * * \\
(0.0462)\end{array}$ & $\begin{array}{c}0.335^{* * *} \\
(0.1144)\end{array}$ & 0.0100 \\
\hline & $p$-val equality test & 0.0422 & 0.7633 & 0.0986 & \\
\hline Envy_others & Female & $\begin{array}{c}0.225^{*} \\
(0.1343)\end{array}$ & $\begin{array}{c}0.380 \\
(0.2404)\end{array}$ & $\begin{array}{c}0.595^{*} \\
(0.3428)\end{array}$ & \\
\hline
\end{tabular}

Note: Standard Errors in parenthesis; *,** and *** indicate 10, 5 and 1 per cent significance levels, respectively. $P$-val equality test (in italics) indicate $p$-value of ta test for the equality of the predicted values across subsamples - the test statistics is distributed $\chi^{2}{ }_{(1)}$. 
The first column of Table 3 show that the predicted number of yogurt packages with healthenhancing features purchased by level of body image dissatisfaction and gender. The second and third columns report the predicted values conditional on the level of body image dissatisfaction, frequency of reading the nutrition label, and gender. The first panel presents the predicted values for respondents declaring to "Like" their own body image; the second panel for those who declare to accept their own body image ("Accept"); the third and fourth panels for respondents who do not like ("Do_Not_Like") their body image or envy others' ("Envy_others"). Also, we performed equality tests for the predicted values across each subsample pair ("Male" vs. "Female", and "Reading Label" vs. "Not Reading Labels"), to assess whether the predicted number of functional yogurt packages purchased differs across subsamples.

The values in Table 3 indicate that the predicted number of packages of functional yogurts purchased declines as respondents experience lower levels of body image satisfaction, regardless on their gender. For male respondents, the predicted values of functional yogurt packaged purchased vary from 0.927 units for respondents who like their own body image to 0.191 for those who declared not to like it. For female respondents, such decline is even steeper, going from 1.451 units for those who like their own body image to 0.225 , for those who envy others'. Thus, this result is in line with other research indicating that being dissatisfied own body image affects negatively individual's food choices and the willingness to engage in long-term healthy eating behaviors (Johnson \& Wardle, 2005; Wardle et al., 2006) which in our case is proxied by the purchase of health-enhancing yogurts. It should be noted that the group of respondents who declared not to like their own body and envy other peoples' is composed exclusively of women (bottom panel of Table 3). Such results confirm other literature that found women to experience a lower level of body satisfaction compared to men (Wardle et al., 2006).

Another pattern that emerges in table 3 is that women are predicted to purchase systematically higher numbers of functional yogurt packages compared to men, across all body image dissatisfaction levels. However, such gender difference is not observed among respondents who do not read food labels when purchasing food (see the large equality test $p$-values in the second column), but shows marked gender differences among respondents who read the nutrition labels, however the gaps become smaller as the level of body image dissatisfaction increases.

Also, we find statistically higher predicted numbers of functional yogurts purchased for those respondents who read the nutrition label "Always", or "Sometimes" when purchasing food, compared to those who never read it, across all levels of body image dissatisfaction and gender, with the exception of male respondents who like their own body image (the p-value of the equality test is 0.1139 , failing to reject the null of the predicted values being statistically the same across male label readers and non-label readers who like their own body image).

Last, we predicted the number of functional yogurt packages at different levels of body image dissatisfaction conditional on the respondent's level of knowledge for leading functional 
yogurt brands, depicted in Figure 3. The graph in Figure 3 shows that regardless on the individual level of knowledge of leading functional brands, respondents who declared not to like their own body, or to envy others' ("Do_Not_Like" and "Envy_Others" lines, respectively) show lower predicted numbers of health-enhancing yogurt packages purchased than respondents declaring to like or accept their own body. Indeed, respondents who like, or accept, their own body image are predicted to buy from two to three times more packages of functional yogurts compared to respondents who experience lower levels of body satisfaction. Similarly, the model predicts that respondents who accept their body image (line "Accept") will purchase fewer numbers of functional yogurt packages compared to those who like their own body (line "Like") at all levels of functional yogurts knowledge. For example, our model predicts that respondent who like their own body will purchase one or more packages of health-enhancing yogurts for levels of functional yogurts knowledge above 2.2, whereas a level of knowledge above average (6.3 or higher) is necessary for those respondents accepting their body image to purchase one package of functional product.

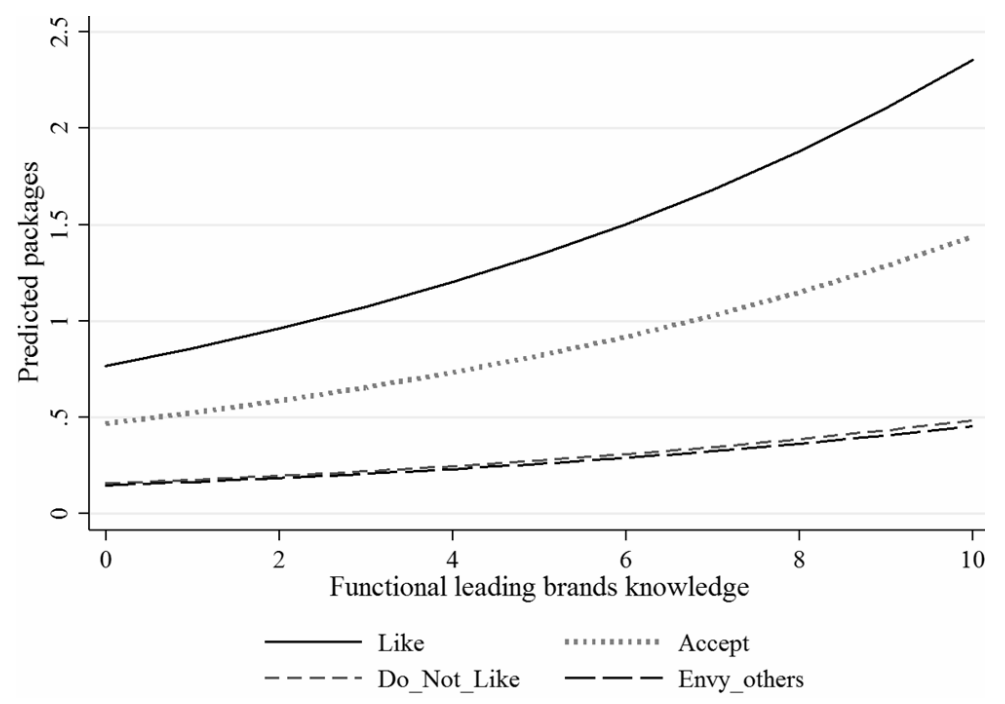

Figure 3. Predicted number of functional yogurt packages purchased conditional body image dissatisfaction and knowledge in leading functional yogurt brands.

\section{General discussion}

The results presented in Table 2 confirm that the feeling of own-body image dissatisfaction acts as a psychological barrier to selecting food with a long-term beneficial effect on health (Johnson and Wardle, 2005; Goldfield et al., 2010). These findings support the hypothesis that individuals who undertake autonomous actions are more likely to select healthier food choices compared to others, consistent with SDT, and are line with other literature indicating a positive relationship between brand familiarity, reading the nutrition label and the selection of healthier food options (Drichoutis et al., 2006; Stranieri et al., 2010; Bimbo et al, 2017). 
The results of our model are also overall consistent with previous findings, also considering the other covariates included in the model. Indeed, women have been indicated as being more health-oriented and thus, being more likely to select healthier food versions over regular ones (Wardle et al., 2004; Drichoutis et al., 2006). Previous research has also found that female consumers have more positive attitudes towards healthier versions of dairy products than men (Johansen et al., 2011) as they prefer, or are willing to try, health-enhancing dairy food versions over conventional ones (Ares and Gambaro, 2007; Ares et al., 2009; Annunziata and Vecchio, 2013) more than man. Also, consumers with relatively high income are indicated as the main users of functional products (Siró et al., 2008) since their purchase behavior is affected by their positive attitude towards own health (Darmon \& Drewnowski, 2008) as well as those consumers living in large household size (Barenna \& Sánchez, 2010). However, contrary to most of the existing literature (Siró et al, 2008; Marette et al., 2010), we find that being affected by chronic diseases is not associated with purchase more functional yogurts packages. The negative relationship between practicing sport and functional yogurt purchases is supported by other literature, as sport may be seen as a substitute to consuming healthenhancing foods, similar to what Landström et al. (2009) found studying the consumption of functional foods in a sample of Swedish consumers.

The predicted values presented in Table 3 are meant to test whether our respondents' behave in a manner consistent with SDT. According to SDT, individuals who undertake selfdetermined actions (e.g. use nutrition label when selecting food) have healthier behaviors regardless the level of body dissatisfaction they experience. Furthermore, women, who generally suffer from a lower level of body satisfaction compared to men, should be at the same time more effective in mitigating their sense of body dissatisfaction (Wardle, Haase, \& Steptoe, 2006; Algars et al., 2009) as well as more likely to engage in self-determined behaviors and adopt long-term healthier eating habits (Parmenter et al., 2000; Pelletier et al., 2004a; Drichoutis et al., 2006; Pelletier \& Dion, 2007). Thus, we expected the predicted number of health-enhancing yogurts purchased by participants to decrease with the level of body image dissatisfaction, and that such reduction is less severe if respondents use frequently nutrition food labels and to be more mitigated for female compared to male respondents.

\section{Conclusions}

In this paper, we provide an empirical assessment of the relationship between consumers' level of body-image dissatisfaction and the number of health-enhancing yogurt versions chosen from a virtual supermarket shelf. Our findings indicate that the number of healthenhancing products that a consumer purchase decreases as her or his level of body image dissatisfaction increases, consistently with the evidence available in the literature finding a negative correlation between body image dissatisfaction and healthy eating habits. Furthermore, our results are consistent with previous findings anchored in Self-Determination Theory (SDT): we find that women are more likely to select healthier food options if their food choices are driven by their interest in food nutritional content and their attitude to undertake more autonomous actions. 
Thus, our analysis highlights the existence of complex mechanisms affecting healthy food choices, and how psychological factors which have so far had little attention in the food marketing literature, that is, the level of body image dissatisfaction, can play an important role in affecting consumers' choice and purchase decisions, especially when it comes to health enhancing food products. Additionally, in this analysis we used a virtual based data collection method, which represents an innovative method to gain insights into consumer behavior. Furthermore, as the data collected using virtual shelves or stores are highly correlated with actual sales data, this data collection method also allows to simulate real-life shopping behavior more closely than survey-based data.

In spite of our contributions, our analysis shows two main limitations. First, it does not explore how the feeling of dissatisfaction for own body image is directly or indirectly affected by internal factors (e.g. attitudes towards health) or external ones (e.g. peers' and societal pressures) and how such factors modulate individual food choices either directly or indirectly through body image dissatisfaction. Thus, future research, using mediation analysis, seem to be needed to explain the mechanisms through which body image affects individuals' food choices conditionally on the role played by internal and external factors in mitigating/magnifying the relationship between body image and healthy food choices. Second, our analysis is limited in scope as it only assesses the relationship between the level of body-image dissatisfaction and the number of health-enhancing options purchased limited to one food category, yogurt. As consumers' preferences for food products may be inherently different across product categories, our results' generalizability may be limited. Therefore, future research should investigate the relationship between the level of body image satisfaction and choice of healthy alternatives across food categories.

\section{References}

Algars, M., Santtila, P., Varjonen, M., Witting, K., Johansson, A., Jern, P., \& Sandnabba, N. (2009). The adult body: how age, gender, and body mass index are related to body image. Journal of Aging and Health, 21(8), 1112-1132.

Annunziata, A., \& Vecchio, R. (2013). Consumer perception of functional foods: A conjoint analysis with probiotics. Food Quality and Preference, 28, 348-355.

Ares, G., \& Deliza, R. (2010). Identifying important package features of milk desserts using free listing and word association. Food Quality and Preference, 21, 621-628.

Ares, G., \& Gámbaro, A. (2007). Influence of gender, age and motives underlying food choice on perceived healthiness and willingness to try functional foods. Appetite, 49, 148158 .

Ares, G., Giménez, A., \& Gámbaro, A. (2009). Consumer perceived healthiness and willingness to try functional milk desserts. Influence of ingredient, ingredient name and health claim. Food Quality and Preference, 20, 50-56. 
Barrena, R., \& Sánchez, M. (2010). The link between household structure and the level of abstraction in the purchase decision process: an analysis using a functional food. Agribusiness, 26, 243-264.

Bimbo, F., Bonanno, A., \& Viscecchia, R. (2016). Do health claims add value? The role of functionality, effectiveness and brand. European Review of Agricultural Economics, 43(5), 761-780.

Bimbo, F., Bonanno, A., Nocella, G., Viscecchia, R., Nardone, G., De Devitiis, B., \& Carlucci, D. (2017). Consumers' acceptance and preferences for nutrition-modified and functional dairy products: A systematic review. Appetite, 113, 141-154.

Bonanno, A. (2012). Some like it healthy: demand for functional and conventional yogurts in the Italian market. Agribusiness, 28(1), 67-85.

Burke, R. R., Harlam, B. A., Kahn, B. E., \& Lodish, L. M. (1992). Comparing dynamic consumer choice in real and computer-simulated environments. Journal of Consumer Research, 19(1), 71-82.

Burke, R. R. (1996). Virtual shopping: Breakthrough in marketing research. The Journal of Product Innovation Management, 6(13), 558-559.

Cameron, A. C., \& Trivedi, P. K. (1990). Regression-based tests for overdispersion in the Poisson model. Journal of Econometrics, 46(3), 347-364.

Campo, K., Gijsbrechts, E., \& Guerra, F. (1999). Computer simulated shopping experiments for analyzing dynamic purchasing patterns: Validation and guidelines. Journal of Empirical Generalisations in Marketing Science, 4(2), 22-61.

Darmon, N., \& Drewnowski, A. (2008). Does social class predict diet quality?. The American journal of clinical nutrition, 87(5), 1107-1117.

Deci, E. L., \& Ryan, R. M. (1985). Intrinsic motivation and self-determination in human behavior. New York, NY: Plenum Press.

Deci, E. L., \& Ryan, R. M. (1987). The support of autonomy and the control of behavior. Journal of Personality and Social Psychology, 53, 1024-1037.

Deci, E. L., \& Ryan, R. M. (2002). Overview of self-determination theory: An organismic dialectical perspective. In E. L. Deci \& R. M. Ryan (Eds.), Handbook of self-determination research. Rochester, NY: University of Rochester Press.

Drichoutis, A. C., Panagiotis L., \& Nayga, R.M.Jr. (2006). Consumers' use of nutrition labels: a review of research studies and issues. Academy of marketing science review, 9, 1-22.

Goldfield, G. S., Moore, C., Henderson, K., Buchholz, A., Obeid, N., \& Flament, M. F. (2010). Body Dissatisfaction, DietaryRestraint, Depression, and Weight Status in Adolescents. Journal of School Health, 80(4), 186-192.

Grigg, M., Bowman, J., \& Redman, S. (1996). Disordered eating and unhealthy weight reduction practices among adolescent females. Preventive medicine, 25(6), 748-756. 
Hailu, G., Boecker, A., Henson, S., \& Cranfield, J. (2009). Consumer valuation of functional foods and nutraceuticals in Canada. A conjoint study using probiotics. Appetite, 52, 257-265.

Johnson, F., \& Wardle, J. (2005). Dietary Restraint, Body Dissatisfaction, and Psychological Distress: A Prospective Analysis. Journal of Abnormal Psychology, 114(1), 119-125.

Krutulyte, R., Grunert, K. G., Scholderer, J., Lähteenmäki, L., Hagemann, K. S., Elgaard, P., Nielsen, B., \& Graverholt, J. P. (2011). Perceived fit of different combinations of carriers and functional ingredients and its effect on purchase intention. Food Quality and Preference, 22(1), 11-16.

Krutulyte, R., Grunert, K.G., Scholderer, J., Hagemanna, K.S., Elgaarda, P., Nielsena, B., \& Graverholt, J.P. (2008). Motivational factors for consuming omega-3 PUFAs: An exploratory study with Danish consumers. Appetite, 5(1), 137-147.

Landström, E., Hursti, U. K. K., \& Magnusson, M. (2009). Functional foods compensate for an unhealthy lifestyle. Some Swedish consumers' impressions and perceived need of functional foods. Appetite, 53(1), 34-43.

Long, J. S., \& Freese, J. (2006). Regression models for categorical dependent variables using Stata. Stata press.

Marette, S., Roosen, J., Blanchemanche, S., \& Feinblatt-Mélèze, E. (2010). Functional food, uncertainty and consumers' choices: A lab experiment with enriched yoghurts for lowering cholesterol. Food Policy, 35, 419-428.

Markey, C.N., August, K.J., Bailey, L.C., Markey, P.M. \& Nave, C.S. (2016). The pivotal role of psychology in a comprehensive theory of obesity. Health Psychology Open, 3(1), 1-4.

Mask, L. \& Blanchard, C.M. (2010). The protective role of general self-determination against 'thin ideal' media exposure on women's body image and eating related concerns. Journal of Health psychology., 16(3): 489-499.

Miller, L., Soederberg, M., \& Cassady, L.D. (2015). The effects of nutrition knowledge on food label use. A review of the literature. Appetite, 92, 207-216.

Neumark-Sztainer, D., Levine, M. P., Paxton, S. J., Smolak, L., Piran, N., \& Wertheim, E. H. (2006). Prevention of body dissatisfaction and disordered eating: What next? Eating disorders, 14(4), 265-285.

Ogden, J. (2012). Health psychology. McGraw-Hill Education (UK).

Parmenter, K., Waller, J., \& Wardle, J. (2000). Demographic variation in nutrition knowledge in England. Health education research, 15(2), 163-174.

Pelletier, L. G., Dion, S. C., Slovinec-D'Angelo, M., \& Reid, R. (2004a). Why do you regulate what you eat? Relationships between forms of regulation, eating behaviors, sustained dietary behavior change, and psychological adjustment. Motivation and Emotion, 28(3), 245277.

Pelletier, L.G., \& Dion, S.C. (2007). An examination of general and specific motivational 
mechanisms for the relations between body dissatisfaction and eating behaviors. Journal of Social and Clinical Psychology, 3, 303-333.

Pelletier, L.G., Dion, S.C., \& Levesque, C.S. (2004b). Can self-determination help protect women against sociocultural influences about body image and reduce their risk of experiencing bulimic symptoms?. Journal of Social and Clinical Psychology, 23, 61-88.

Siegrist, M., Stampfli, N., \& Kastenholz, H. (2008). Consumers' willingness to buy functional foods. The influence of carrier, benefit and trust. Appetite, 51, 526-529.

Simonson, I. (1990). The effect of purchase quantity and timing on variety-seeking behavior. Journal of Marketing Research, 27, 150-162.

Siro, I., Kapolna, E., Kápolna, B., \& Lugasi, A. (2008). Functional food. Product development, marketing and consumer acceptance-A review. Appetite, 51(3), 456-467.

Stranieri, S., Baldi, L., \& Banterle, A. (2010). Do nutrition claims matter to consumers? An empirical analysis considering European requirements. Journal of Agricultural Economics, 61(1), 15-33.

Thogersen-Ntoumani, C., Ntoumanis, N., \& Nikitaras, N. (2010). Unhealthy weight control behaviours in adolescent girls: A process model based on self-determination theory. Psychology and Health, 25(5), 535-550.

Van Kleef, E., van Trijp, H. C., \& Luning, P. (2005). Functional foods: health claim-food product compatibility and the impact of health claim framing on consumer evaluation. Appetite, 44(3), 299-308.

van Herpen, E., van den Broek, E., van Trijp, H.C.M., \& Yu, T. (2016). Can a virtual supermarket bring realism into the lab? Comparing shopping behavior using virtual and pictorial store representations to behavior in a physical store. Appetite, 107, 196-207.

Verstuyf J, Vansteenkiste, M., \& Soenens B. (2012a). Eating regulation and bulimic symptoms: The differential correlates of health-focused and appearance focused eating regulation. Body Image, 9, 108-117.

Verstuyf, J., Patrick, H., Vansteenkiste, M., \& Teixeira, P.J. (2012b). Motivational dynamics of eating regulation: a self-determination theory perspective. The International Journal of Behavioral Nutrition and Physical Activity, 9, 21-37.

Wardle, J., Haase, A. M., \& Steptoe, A. (2006). Body image and weight control in young adults: International comparisons in university students from 22 countries. International Journal of Obesity, 30(4), 644-651.

Wardle, J., Haase, A. M., Steptoe, A., Nillapun, M., Jonwutiwes, K., \& Bellisie, F. (2004). Gender differences in food choice: the contribution of health beliefs and dieting. Annals of Behavioral Medicine, 27(2), 107-116. 
Chapter 6

General Discussion 


\subsection{Introduction}

In recent years policymakers have aimed to increase the general awareness regarding the link between diet and health (Traill, 2012), providing food companies an incentive to differentiate their products by adding health-enhancing features (Réquillart and Soler, 2014). The European market of these products, referred to here as functional foods, is regulated according to the European regulation 1924/2006 on nutrition and health claims on foods (Reg. (EC) No.1924/2006).

Even though the European functional foods' market has grown steadily over the last 20 years (Research and Markets, 2014), more than 3 out of 4 new functional products are withdrawn from the market within two years from launch (Stein and Rodriguez-Cerezo, 2008). One of the possible reasons for such high failure rate is that consumer acceptance of, and preference for functional products are not appropriately taken into account during the product development phase (Bleiel, 2010). Such lack of coordination between product development and consumer demand, leads to a mismatch between consumers' needs and the features of new functional food products introduced in the market (Van Kleef et al., 2002). Furthermore, the existing research on consumers' acceptance of, and preferences for functional foods (Lähteenmäki, 2013; Kaur and Singh, 2017) is fragmented, and presents heterogeneous results (Starling, 2014).

An additional hurdle for firms attempting to differentiate their products by adding healthenhancing features is the risk of experiencing low returns on those investments necessary to produce and market functional products, because of the uncertainty regarding receiving prices high enough to remunerate the investments. In fact, studies of consumers' willingness to pay (henceforth WTP) for functional food products show mixed results: while some of them found a high WTP for functional attributes in food products (Ding et al., 2012; Balcombe et al., 2014; Moro et al., 2015); others instead, found that only a limited premium price can be achieved (Menrad, 2003; Larue et al., 2004).

The general objective of this thesis is to assess quantitatively the implicit price of health claims as well as to generate new knowledge that will inform firms on how to target consumers with high WTP.

Specifically, Chapter 2 consists of a systematic review of the literature on consumer acceptance of, and preference for nutrition-modified and functional dairy products, focusing on those factors that affect consumer acceptance of health claims. Chapter 3 estimates the implicit price of different health claims available in the Italian yogurt market and the heterogeneity of their implicit prices based on three factors identified in Chapter 2: health claims' type, efficacy, and brand familiarity. Health claims' implicit prices are estimated using a hedonic price model (Rosen, 1974) applied to a large dataset of yogurt sales in Italy. Chapter 4 explores manufacturers' pricing ability allowing for asymmetric information on buyer's side. To this end Chapter 4 uses Kumbhakar and Parameter's (2010) extension of the traditional hedonic price model, which relaxes the full information assumption, to a database derived from that used in Chapter 3. Last, Chapter 5 uses a novel data collection method, a virtual shelf shopping experience (Burke, 1996), to overcome one of the limitations of sales data, that is, the lack of 
information on consumers, including psychological traits. These data ae used to assess how the number of functional yogurt alternatives chosen is linked to respondents' psychological traits (i.e. body image dissatisfaction), familiarity with the functional leading brands, and other consumer characteristics.

The remainder of this chapter synthesizes first the results illustrated in this thesis (Section 6.2). Then, it will illustrate the theoretical frameworks, methodological approaches and data used (Section 6.3), followed by a discussion of policy and business implications (Section 6.4 and 6.5) and future avenues of research (Section 6.6). The chapter ends illustrating the main conclusions of the thesis (Section 6.7).

\subsection{Synthesis of results}

This thesis addresses two key issues, which are the estimation of implicit price of health claims allowed by the European Regulation No.1924/2006, and creating new knowledge to inform firms on how to target consumers with high WTP for the bundle of attributes they offer.

Scholars from marketing and consumer sciences have studied consumer's acceptance of, and preference for functional foods as these are key factors for the market success of functional products (Verbeke, 2005). Chapter 2 provides a systematic review of the existing literature on consumer acceptance of, and preference for nutrition-modified and functional dairy products published in peer review journals during the period from 1999 to 2013. The findings of the selected studies confirm those of other literature reviews on how consumers' characteristics and a product's intrinsic attributes influence consumers' acceptance of, and preference for nutritionmodified and functional foods. Chapter 2 results are in line with those of other literature reviews (e.g. Sirò et al., 2008; Lähteenmäki, 2013) confirming that extrinsic product attributes, such as health benefit type (in the case of yogurts, these are cholesterol reduction, support for the immune system, promoting bowel regularity, and bone health) and the claimed effectiveness on human health (disease risk reduction vs. general functional activity) affect consumer acceptance of, and preferences for products with health claims. The study reviewed in Chapter 2 present the new insight that functional foods acceptance depends also upon consumers' familiarity with the brand name associated with the health claim. The way brands affect consumers' evaluation of nutrition-modified and functional products has not been highlighted in any other prior literature review.

Chapter 3 assesses the implicit price of health claims allowed by the European Regulation No.1924/2006. Using the findings of Chapter 2, the estimated implicit price of each health claim is conditioned on three factors: the type of health benefit provided, the guaranteed level of efficacy on human health, and whether a claim is supported by a familiar brand. The econometric estimates show different values associated with the different claimed benefits. The claims with the highest implicit prices are those lowering/managing cholesterol blood level, and those supporting bone health. The results also suggest that a claim's efficacy matters in determining a product's premium price: in the case of cholesterol lowering products, disease risk reduction claims have a much larger implicit price than general function ones. Furthermore, most health 
claims supported by a familiar brand benefit from a higher implicit price than those supported by less familiar ones. Thus, using actual sales data, the results from Chapter 3 provide empirical support to the findings of the systematic literature review discussed in Chapter 2.

Chapter 4 explores whether manufactures' investments in credence attributes such as, health claims, "organic", and "natural" claims ${ }^{1}$, enable food manufacturers to reach systematically consumers with higher WTP for the attribute bundles they offer. For credence attributes, whose presence cannot be assessed a priori, asymmetric information may result in consumers not being willing to pay a high price for products carrying them, which may impair manufacturers' ability to differentiate their products. The findings of Chapter 4 indicate that manufacturers with a larger portfolio of products carrying credence attributes can systematically reach consumers with a higher WTP for their products. Such result suggests that introducing products with credence attributes, such as health claims or using "organic" or "natural" claims in a producer's portfolio, may increase the extent of product differentiation, and result in an improved reputation. Thus, firms which invest strategically in credence features may be able to segment the market more effectively than other firms and reach consumers with a higher WTP.

Chapter 5 explores the relationship between the choice of purchasing functional alternatives, knowledge about leading functional brands, and one's level of satisfaction with his/her own body image. In other words Chapter 5 creates a framework to validate and expand upon the findings of the literature reviewed in Chapter 2, assessing the synergic effect of a psychological trait and consumer brand knowledge on functional alternatives' choice. In fact, the consumer literature on functional foods has not explored yet the role of body image satisfaction, even though this has been found correlated to a diet's healthfulness (Verstuyf et al., 2012; Markey et al., 2016); this branch of the literature finds that being unsatisfied with own body image is associated with longterm unhealthy eating behaviour, and a lower selection of healthy foods. Chapter 5 tests the hypothesis that the lower the level of own body image satisfaction, the smaller the purchased quantity of functional options is. Furthermore, it measures how the positive effect of functional brand knowledge affects the number of functional alternatives purchased conditionally on body image dissatisfaction levels. The findings presented in Chapter 5 indicate that the level of own body image dissatisfaction is negatively correlated with the likelihood of selecting functional options from a virtual supermarket shelf, that the magnitude of such relationship increases with the level of body dissatisfaction, and that this declines with the functional brand knowledge.

Summarizing, this thesis estimates the implicit price of health claims allowed by the European Regulation No.1924/2006 available on the market (Chapter 3) conditional on factors affecting consumer acceptance: the type of health benefit provided, the guaranteed level of efficacy on human health, and whether a claim is supported by a familiar brand (Chapter 2). Furthermore, it explores whether investing in a larger number of health claims, or credence attributes in general, can enable a firm to target consumers with the highest WTP in a market where the presence of asymmetric information and consumer's distrust may hinder such ability (Chapter 4). Lastly, this thesis explores how a psychological trait, the level of body image satisfaction along with other

\footnotetext{
${ }^{1}$ Premium price for attributes as "Organic" and "Natural", estimated in Chapter 3, is higher than those of many "General function" health claims.
} 
factors (i.e. functional brand knowledge) are related to the consumers propensity to choose more functional options within a category, using a novel data collection method, a virtual supermarket shelf (Chapter 5).

\subsection{Theoretical frameworks, methodological approaches and data}

A variety of theoretical frameworks, methods and data were used to achieve the specific objectives of this thesis. This section presents a brief discussion of each of them.

\subsubsection{Theoretical Frameworks}

Different theoretical frameworks guided the research in this Thesis. The review of the literature in Chapter 2 showed the existence of heterogeneous conceptual frameworks guiding research on assessing consumer choice of functional foods. Some of these approaches are based on the psychology of food choices (e.g. protection motivation theory, theory of planned behaviour, food neophobia, mere-exposure theory, etc.) whereas others in micro-economic theory. The analyses in Chapter 3 and Chapter 4 are based on a model rooted in microeconomic theory, the hedonic price model, whereas that in Chapter 5, uses a conceptual framework recently proposed in consumer science to investigate food choices, self-determination theory.

Chapter 3 and 4 use hedonic price models. According to hedonic price theory, a product is considered as a bundle of attributes. Each consumer chooses that bundle of attributes maximizing his/her utility, subject to a budget constraint. Likewise, manufacturers maximize profits by setting a product's price according to the attributes it contains conditionally on the technology available (Rosen, 1974). In a market with products containing unique bundles of attributes, the marginal bids of buyers and the marginal offers of sellers match at the equilibrium. The joint envelope of bid curves (from demand) and offer curves (from supply) generates the hedonic price function (Rosen, 1974), where the price of a product is equal to the sum of the implicit prices of its characteristics.

While the analysis preformed in Chapter 3 follows the "traditional" hedonic price model, Chapter 4 uses an extension of this approach, relaxing one of the assumptions of Rosen's framework, that is, that of both groups of agents in the market (buyers and sellers) being perfectly informed. Kumbhakar and Parameter's (2010) extension of the hedonic price model considers that, in markets where there exist asymmetric information, price dispersion may emerge and producers may not be able to target consumers with high WTP, and/or buyers may not find sellers offering products at low values of Willingness To Accept (WTA), generating multiple hedonic price curves. Chapter 4 uses a restricted version of Kumbhakar and Parameter's (2010) framework to assess producers' ability to target consumers with the highest WTP.

The conceptual framework the analysis performed in Chapter 5 is based on, is the selfdetermination theory. Self-determination theory is a wide reaching conceptual framework to assess multiple facets of human behaviour, including, inter alia exercise, work and education (Vansteenkiste, Neimec and Soenens, 2010; Vestruyf et al., 2012). According to this theory there is a series of factors, such as internal motivations, external stimuli, heterogeneous responses, 
needs and goals, driving different aspects of human behaviour. Recently, Vestruyf et al. (2012) argue that self-determination theory is a suitable framework to assess motivations in eating regulation (i.e. the series of behaviours and goals affecting eating), which applies to healthy eating and the mechanisms that regulate it. Consistently with other theories, self-determination theory links issues of body image to the likelihood of adopting long-term healthy eating behaviours.

\subsubsection{Methodological Approaches}

Chapter 2 uses a systematic literature review technique to identify consumer-related factors affecting acceptance of, and preference for nutrition-modified and functional dairy foods. The systematic literature review differs from the narrative literature review as it has one additional step: the quality assessment of the findings. The quality assessment step entails designing a protocol to establish the quality of the studies surveys, and then it weights findings according to the quality of the study they originate from (Cooper, 1984). This step allows researchers to organize and discuss results from other studies accounting for their quality, and to provide an integrated picture of the subject in analysis (Cooper, 1984). In Chapter 2 the quality assessment is performed using an ad hoc protocol for social science research (Littell et al., 2008).

Chapter 3 assesses the implicit price of health claims allowed by Reg. No.1924/2006, conditional on three factors affecting consumers' acceptance (as highlighted in Chapter 2). To achieve this goal, it uses a standard hedonic price model, as proposed by Rosen (1974). The analysis performed in Chapter 3 estimates the parameters of the hedonic price function using a singleequation approach (Carlucci et al., 2013; Szathvary and Trestini, 2013; Loke et al., 2015) to determine the implicit price of the product attributes. The model is estimated via ordinary least squares (OLS), and the appropriate functional form is selected following the approach illustrated in Costanigro, McCluskey, and Mittelhammer (2007).

Chapter 4 estimates an extension of the hedonic price model following Kumbhakar and Parameter's (2010) approach. The estimation method employed in Chapter 4 is a maximum likelihood stochastic frontier estimator. The estimates allow to test whether the presence of a larger number of credence attributes (health claims, organic and natural claims) in a producer's portfolio reduces the distance of a manufacturers' products prices from the hedonic curve representing consumers' highest WTP.

Chapter 5 uses count-data maximum likelihood estimation procedures to study how the number of functional yogurt packages selected by the participants from a virtual supermarket shelf relates to respondents' level of body image dissatisfaction, their familiarity with leading functional brands, and their socio-demographic characteristics. Tests to identify the proper distribution to be used (e.g. poisson, negative binomial, and zero-inflated negative binomial) are performed. The best fitting model (negative binomial) is used to determine the shape of the relationship between the number of functional alternatives chosen and body-image dissatisfaction, conditional on the level of functional brands familiarity. 


\subsubsection{Data}

The data used in this thesis are heterogeneous across chapters: Chapter 2 analyses systematically the findings of other studies; Chapter 3 and 4 use sales data and Chapter 5 uses data collected via a virtual shelf shopping experience.

Most of the studies selected in chapter 2 were cross-sectional in nature and showed a large variation in term of study design and methodological approach. In most cases, the studies reviewed investigated consumer acceptance of, and preferences for nutrition-modified and functional products using different scales, ${ }^{2}$ product concepts instead of actual products, and focused on small samples of respondents in limited geographical areas (mostly Northern European countries, few Southern European and American). Thus, the findings reviewed showed ample variation, low comparability and may be affected by hypothetical bias due to the survey nature of the studies (Lusk et al., 2004).

Chapter 3 and 4 attempted to overcome some of the shortcomings illustrated above by using actual regional, monthly sales of yogurt collected at the point-of-sale in all Italian Regions. The scanner data, originally from SymphonyIRI Group (courtesy of the University of Foggia, Italy) were recorded at the European Article Number (EAN) level ${ }^{3}$ allowing to observe monthly volumes sold, value of sales, price (EUR/L) as well as several product characteristics. Thus, the analysis performed in Chapter 3 and 4 used a "revealed preference" approach instead of a "stated preference" one.

Chapter 5 used instead a novel method for data collection, simulating a real supermarket shelf, which generates data highly correlated with real sales. The data collected allow to capture respondents' personal characteristics, including psychological and behavioural traits, information which is usually lacking in sales data, such as those used in Chapter 3 and 4 . Thus, the virtual shelf method has the advantage of allowing testing of new and emerging consumer theories, and to generate findings having higher external validity compared to those attainable using surveys, which are more likely to suffer from hypothetical bias.

\subsection{Policy implications}

The European Regulation (EC) No 1924/2006 on nutrition and health claims made on foods sets the criteria for products' health claims approval, classifying them mainly into two different categories: "Reduction of disease risk" claims (Article 13) and "General function" claims (Article 14).

Firms that want to use "General function" claims (Article 13) are authorized on the basis of generally accepted scientific evidence and are not required to submit an individual application for authorization, as required for "Reduction of disease risk" claims (Article 14). The rationale for having a generic list of 'general function' claims approved on the basis of generally accepted

\footnotetext{
${ }^{2}$ See footnote 3 in Chapter 1 for more details.

${ }^{3}$ The EAN barcodes are 13-digit codes used in the coding system at the retail level in Europe for identifying products by scanner cash registers at the point of sale where products are sold
} 
scientific evidence, was to enable small- and medium-size food companies to use health claims without having to submit an application for full authorization, given that such process is costly and time consuming (Brooke, 2010). However, although Article 13 of Regulation (EC) No.1924/2006 may encourage small- and medium-size food companies to differentiate their products by adding "General function" claims, there is a risk that such regulatory scheme may lead to a market proliferation of similar products and ultimately nullify the firms' efforts to product differentiate (Bröring et al., 2016).

Food companies wanting to use "Reduction of disease risk claim", are required to submit individual dossiers (applications for authorization) supporting the specific claim. Applications then undergo a case-by-case assessment by an EFSA panel. Such authorisation mechanism has been found to be costly, uncertain, as well as lengthy, if compared to regulatory schemes of other countries (Brooke, 2016). ${ }^{4}$ The estimated costs to produce evidence in support of the health claim application, and the causal relationship between the active principle and its claimed effect on human health, vary from 0.25 to 1 million EUR plus. Also, despite the high investments necessary to create each dossier, less than 1 out of 4 "Reduction of disease risk" dossiers submitted has been approved by the EFSA (Brooke, 2016). Furthermore, firms denounce a lack of guidance on how to prepare dossiers and on what types of studies should be included to increase the likelihood of their acceptance (Brooke, 2016). Also, considering only those dossiers with a positive outcome, their approval time ranges from fifteen months to four and a half years, with an average time of about two-and-a-half years (Brooke, 2016).

Thus, large food companies are more likely than smaller ones to be able to afford the economic burden of investing in a "Reduction of disease risk" claim. Indeed, a product differentiation strategy based on using "Reduction of disease risk" claim can be prohibitively costly for many European food companies, as they are mainly of small and medium size with traditionally low level of $R \& D$ investment ( $<1 \%$ of sales) and short $R \& D$ cycles ( $1-3$ years) (Banterle et al. 2011). Although this thesis does not formally account for the size of firms, the database used in Chapters 3 and 4 offers some insights on the availability of products with "Reduction of disease risk" in a specific market (the Italian yogurt market). Considering the manufacturers analysed, only one out of twenty-three companies marketed a product carrying a "Reduction of disease risk" claim; however, that claim was the one with the highest implicit price among all health claims analysed (Chapter 3). Also, the same company has in its portfolio products carrying all of the four "General function" claims available on the market, and, based on the results of Chapter 4 , it may have positioned itself optimally to target consumers with the highest WTP. Instead, less than half of the food companies appearing in the data, (eleven out of twenty-three), marketed at least one product with "General function" claims, and the share of manufacturers carrying more than one of such claims, was inversely related to the number of "General function" claims in their product portfolio. Thus, using the Italian yogurt market as a case study, less than half of the companies in the data may have benefited from a premium price achieved by using health claims to differentiate their products. In summary, in the present case study, only one food company

${ }^{4}$ At January 2017, 10 years after that Regulation (EC) No.1924/2006 became effective, EFSA authorized 229 "General Function" claims (Article 13.1) while only 25 "Reduction of disease risk claim" ones (Article 14). 
took the most advantage of investments in health claims. This result seems in line with Sutton's (1991) theory, according to which in industries where quality is produced through fixed costs (such as R\&D costs and costs related to the claims' approval), at least one firm will have an incentive to invest in quality to reinforce the oligopolistic nature of the industry where it operates. As a result, the high level of investments, approval uncertainty, and costliness of clinical trials may create barriers to entry in the European functional food market, reducing competition.

Ultimately, the European Regulation (EC) No.1924/2006 may be hindering food companies' willingness to innovate and differentiate their products by adding health-enhancing features. Thus, policymakers should prioritize the provision of greater guidance to firms on how to create dossiers supporting "Reduction of disease risk" claims and lowering the uncertainty related to Article 13 claims' approval. Further, another priority area for policymakers is to reduce the time for dossier approval giving firms the opportunity to begin commercialise their products at an early date and hence begin to receive revenue to repay prior investments.

\subsection{Business implications}

This thesis suggests that, overall, consumers prefer products with health claims over conventional ones (Chapter 2); thus, differentiating food products by adding health enhancing features can be a successful strategy to escape from price competition (Chapter 3 and 4). In proceeding with this discussion, it should be mentioned that it will be based solely on this thesis' findings regarding the ability to charge higher prices, or to reach consumers with higher WTP. As such, this thesis' results do not speak about the costliness of the investments necessary to produce functional products, and more importantly, to guarantee their approval from EFSA. As discussed in Section 6.4, some of these investments can be prohibitively costly and may not be sustainable for small / medium-size food companies.

Based on this thesis' results (Chapter 3 and 4), which are specific to the Italian yogurt market, food companies should be selective in the types of health claims added to their products. In the specific case of yogurt manufacturers, they may prefer to invest in the development of functional products that lower /manage the cholesterol blood level and those supporting bone health, since the implicit prices of these attributes are the highest among those considered (Chapter 3 and 4). Further, manufactures may want to invest in "Reduction of disease risk" claims rather than in "General function" claims, as they may lead to a higher premium (Chapter 3), in line with previous literature which finds that consumers show strong preferences for products aiming to prevent or to reduce the risk of diseases rather than "General function" claims (Chapter 2). Furthermore, firms investing in the development of functional products may need to first invest in building and strengthening their brand image as a means to gain consumers' trust. The results obtained for the Italian yogurt market indicate that claims supported by well-known brands have a higher implicit price compared to those which are not (Chapter 3). Thus, investments in brand building activities should be a priority for firms aiming to succeed in the functional foods market. 
However, as illustrated in Section 6.4, not all food manufacturers are likely to have financial resources to face the high investment costs needed to differentiate their products using health claims. Thus, food companies with limited resources may decide to differentiate their products by using "General function" claims, or alternatively using credence attributes such as "organic" and "natural" (Chapter 3 and 4). These results indicate that the implicit prices of "organic" and "natural" are higher than those of many "General function" claims (Chapter 3 and 4). The cost of compliance with Regulation (EC) 834/2007 that sets the roles for obtaining the organic certification, ranges from 1,553 to 2,717 EUR (Dabbert et al., 2012) which is comparable to that of Regulation (EC) No.1924/2006 for "General function" claims (Brooke, 2010). However, since there is neither a harmonized regulation across European State members concerning the term "natural", nor a well-established definition of what a "natural" claim entails for a food product (Costantino, 2014), small food manufacturers may differentiate their products using this attribute without having to face prohibitive certification costs. In all cases, any food manufacturer adding "credence" features to their products portfolio may be able to reach systematically consumers with higher WTP as they can signal higher quality to consumers (Chapter 4).

Lastly, even manufacturers benefitting by high levels of brand image should be worry of the fact that the likelihood of product success can depend upon a series of consumer's psychological characteristics such as body image dissatisfaction, some of which have not yet been given the appropriate consideration in the marketing literature (Chapter 5).

\subsection{Limitations and Future Research}

In spite of this theses results' usefulness to guide to food manufacturers in deciding whether or not to invest in the development and marketing of specific functional products, this thesis shows three main limitations.

First, the findings illustrated here apply only to the dairy category, and specifically to yogurts, and are limited to one geographic area, the Italian market. The results presented in this thesis should be confirmed by studying other product markets as well as other geographical contexts.

Second, the results in Chapters 3 and 4 were obtained using aggregate market level data and do not explicitly take into account consumers' heterogeneity in the process of price formation, as well as firms' characteristics and specific costs incurred to supply credence attributes. Therefore, future studies could use richer and more granular data containing both consumers' and manufacturers' features to account for their role in the price formation process. Additionally, these Chapters used implicit prices as the metric of a product's success, and did not take into account the role of investment costs to produce / market these products. Future research should properly account for investment costs and model the profitability of functional products, expanding upon other recent research on the topic (e.g. Bonanno, Huang and Liu, 2015).

Third, and last, Chapter 5 was conducted using data collected through a virtual shelf method, whose results benefit from higher external validity than studies using survey data. Furthermore, other data collection methods employing more sophisticated virtual reality technology exist, such 
as the 3D virtual supermarket (Van Herpen et al., 2016). Thus, to ensure an even higher level of accuracy in simulating actual shopping behavior, future research may employ virtual supermarket data collection methods to cross validate findings of survey-based studied (like those reviewed in Chapter 2), those using actual sales data (i.e. Chapter 3 and 4) as well as those employing virtual shelf data collection method. However, data collected via virtual choice experiments share some of the main drawbacks of those collected through surveys, that is their cross-sectional nature, and the limited sample size, limitation also affecting the results discussed in Chapter 5 of this thesis.

\subsection{Main Conclusions}

The main conclusions of this thesis are as follows:

1. A systematic review of the literature finds that consumer related characteristics such as age, gender, nutritional knowledge, and exposure (direct or indirect) to chronic diseases, affect positively the acceptance of, and preference for, nutrition-modified and functional dairy products (Chapter 2);

2. Psychological factors, such as interest in health and perceived self-efficacy, influence positively the acceptance of, and preference for functional dairy products; instead, food neophobia and body image dissatisfaction reduce consumer's acceptance and preference (Chapter 2 and Chapter 5);

3. The systematic literature review highlights a consensus that claims supported by familiar brands, as well as "risk reduction" (vis-à-vis "general function") ones increase consumers' acceptance of functional dairy products; brand familiarity and a claim's effectiveness, lead to higher health claims' implicit prices (Chapter 2 and Chapter 3);

4. In the Italian yogurt market the implicit prices of "risk reduction" claim is higher than the "general function" ones (respectively, $+222.6 \%$ vs $+85.5 \%$ of the baseline alternative's price in the case of cholesterol reduction health claim), and in three out of four cases, health claims supported by familiar brands have higher implicit price that those supported by less-familiar ones (Chapter 3);

5. Manufacturers operating in the Italian yogurt market investing in credence attributes (health claims, organic and natural) gain the ability to target consumers with higher WTP by including two or more products carrying credence attribute in their portfolio (Chapter 4);

6. Natural and organic claims lead to implicit prices which are (approximately) $+46.6 \%$ and $+102.0 \%$ over the price of the baseline alternative (Chapter 3). Such implicit prices are comparable to those of some health claims (Chapter 3 and Chapter 4);

7. Using a virtual shelf choice setting, of a sample of Italian yogurt consumers, reveals that respondents less satisfied with their own body image select a lower number of functional yogurt packages (Chapter 5);

8. Consumers' functional brand familiarity is positively related to the number of functional alternative selected in a virtual shelf shopping experience (Chapter 5). 
Chapter 6

\section{References}

Balcombe, K., Bitzios, M., Fraser, I. and Haddock-Fraser, J. (2014). Using attribute importance rankings within discrete choice experiments: an application to valuing bread attributes. Journal of Agricultural Economics 65(2): 446-462.

Banterle, A., Cavaliere, A., Carraresi, L. and Stranieri, S. (2011). Innovativeness in food small business: What is its relationship with marketing? Agricultural Economic Czech Republic 57: 474-483.

Bimbo F., Bonanno, A. and Viscecchia R. (2016). Do health claims add value? The role of functionality, effectiveness and brand. European Review of Agricultural Economics 43(5): 761780 .

Bonanno A., Huang R., and Liu Y. (2015). Simulating Welfare Effects of Europe's Nutrition and Health Claims regulation: the Italian Yogurt Market. European Review of Agricultural Economics. 42:499-533.

Brookes, G. (2010). Economic Impact Assessment of the European Union (EU)'s Nutrition\& Health Claims Regulation on the EU Food Supplement Sector and Market. European Health Claims Alliance EHCA.

Bröring, S., Khedkar, S. and Ciliberti, S. (2017). Reviewing the Nutrition and Health Claims Regulation (EC) No. 1924/2006: What do we know about its challenges and potential impact on innovation?. International Journal of Food Sciences and Nutrition, 68(1), 1-9.

Burke, R.R. (1996). Virtual shopping: Breakthrough in marketing research. The Journal of Product Innovation Management, 6(13), 558-559.

Carlucci, D., Stasi, A., Nardone, G. and Seccia, A. (2013). Explaining price variability in the Italian yogurt market: a hedonic analysis. Agribusiness 29: 194-206.

Cooper, H. M. (1998). Synthesizing research: A guide for literature reviews: Vol. 2. Applied social research methods (3rd ed.). Thousand Oaks, CA: Sage.

Costanigro, M., McCluskey, J. J. and Mittelhammer, R. C. (2007). Segmenting the wine market based on price: hedonic regression when different prices mean different products. Journal of Agricultural Economics 58: 454-466.

Costantino L., 2014. L'utilizzo del termine "naturale" nell'etichettatura dei prodotti alimentari. Rivista di diritto alimentare. 8(3): 10-17.

Dabbert, S. (2011): Improving the organic certification system: Recommendations from the CERTCOST project. Report D24 available at www.certcost.org and http://www.ifoameu.org/sites/default/files/page/files/ifoameu_reg_regulation_dossier_201204_en.pdf.

Darby, MR. and Karni, E. (1973). Free competition and the optimal amount of fraud. Journal of Law Economics. 16:67-88. 
Ding, Y., Veeman, M. M. and Adamowicz, W. L. (2012). The influence of attribute cutoffs on consumers' choices of a functional food. European Review of Agricultural Economics 39(5): 745-769

Kaur, N., \& Singh, D. P. (2017). Deciphering the consumer behaviour facets of functional foods: A literature review. Appetite, 112: 167-187.

Kumbhakar, S.C. and Parmeter C.F. (2010). Estimation of hedonic price functions with incomplete information. Empirical Economics, 39(1): 1-25.

Lähteenmäki, L. (2013). Claiming health in food products. Food Quality and Preference, 27(2), 196-201.

Littell, J.H. and College, B.M. (2006). Systematic Reviews in the Social Sciences: A Review. Evidence \& Policy, 2(4), 535-537.

Lusk, J.L., Feldkamp, T. and Schroeder, T. C. (2004). Experimental Auction Procedure: Impact on Valuation of Quality Differentiated Goods'. American Journal of Agricultural Economics 86: 389-405.

Markey, C.N., August, K.J., Bailey, L.C., Markey, P.M. \& Nave, C.S. (2016). The pivotal role of psychology in a comprehensive theory of obesity. Health Psychology Open, 3(1), 1-4.

Menrad, K. (2003). Market and marketing of functional food in Europe. Journal of Food Engineering 56(2): 181-188.

Moro, D., Veneziani, M., Sckokai, P. and Castellari, E. (2015). Consumer willingness to pay for catechin enriched yogurt: evidence from a stated choice experiment. Agribusiness: An International Journal 31(2): 243-258.

Regulation of European Commission (EU) No 1924/2006 of the European Parliament and of the Council of 20 December 2006 on nutrition and health claims made on foods. European Commission, EUR 23380 EN. Luxemburg: European Communities.

Réquillart, V. and Soler, L. G. (2014). Is the reduction of chronic diseases related to food consumption in the hands of the food industry?. European Review of Agricultural Economics 41(3): 375-403.

Research and Markets (2014). Global Functional Food and Nutraceuticals Market (2014 - 2020) - By Type (Foods, Beverages, Supplements); Benefits (Health and Wellness, Disease Prevention, Fitness, Beauty); Origin \& Ingredient.

Sutton, John. Sunk costs and market structure: Price competition, advertising, and the evolution of concentration. MIT press, 1991.

Siró, I., Kápolna, E., Kápolna, B. and Lugasi, A. (2008). Functional food. Product development, marketing and consumer acceptance - a review. Appetite 51: 456-467.

Starling, S., (2014). Functional foods resist recession but failure rate stays high: Analyst. July $7^{\text {th }}$. Available at Nutraingredients.com http://www.nutraingredients.com/ConsumerTrends/Functional-foods-resist-recession-but-failure-rate-stays-high-Analyst Retrieved 
Chapter 6

09.04.2015.

Stein, A.J. and Rodriguez-Cerezo E. (2008). European Commission- Joint Research Centre Institute for Prospective Technological Studies. Functional Food in the European Union.

Szathvary, S. and Trestini, S. (2014). A hedonic analysis of nutrition and health claims on fruit beverage products. Journal of Agricultural Economics 65(2): 505-517.

Traill, B.W. (2012). Presidential Address: Economic Perspectives on Nutrition Policy Evaluation. Journal of Agricultural Economics 63(3): 505-527.

Valls, J., Pasamontes, N., Pantaleón, A., Vinaixa, S., Vaqué, M., Soler, A., Milan, S and Gómez, X. (2013). Prospects of functional foods/nutraceuticals and markets. In Natural products (pp. 2491-2525). Springer Berlin Heidelberg.

Vansteenkiste, M., Niemiec, C.P., and Soenens, B. (2010). The decade ahead : theoretical perspectives on motivation and achievement. In Advances in motivation and achievement, 16A:105-166.

Van Kleef, E., van Trijp, H., Luning, P. and Jongen, W.M. (2002). Consumer-oriented functional food development: how well do functional disciplines reflect the 'voice of the consumer'? Trends in Food Science \& Technology 13(3): 93-101.

Van Herpen, E., van den Broek, E., van Trijp, H. C., and Yu, T. (2016). Can a virtual supermarket bring realism into the lab? Comparing shopping behavior using virtual and pictorial store representations to behavior in a physical store. Appetite, 107, 196-207.

Verbeke, W. (2005). Consumer acceptance of functional foods: socio-demographic, cognitive and attitudinal determinants. Food quality and preference 16(1): 45-57.

Verstuyf, J., Patrick, H., Vansteenkiste, M. and Teixeira, P.J. (2012). Motivational dynamics of eating regulation: a self-determination theory perspective. The International Journal of Behavioral Nutrition and Physical Activity, 9, 21-37. 


\section{Summary}

The rising incidence of non-communicable diseases (such as heart stroke, high blood pressure, diabetes, coronary heart disease, and arthritis) led European policymakers to increase the general awareness regarding the link between diet and health by promoting informed consumer choices. This policy action provided an opportunity for food companies to pursue product differentiation strategies by adding health-enhancing features to their products.

However, in spite of the growing interest regarding functional food products, over 75 per cent of new functional products launched in the European market are withdrawn within the first two years from launch. In fact, firms have to overcome three main obstacles to market healthenhanced products successfully. First, consumer-related factors, such as the heterogeneity of consumer acceptance of, and preferences for, new functional products; second, firms' difficulties in selecting the optimal product attribute profiles to meet consumer demand for health features and; third, firms' difficulties to target consumers with a high enough willingness to pay to ensure the profitability of those investments needed to develop and market functional products.

The general objective of this thesis is to assess quantitatively the implicit price of health claims, and to generate new knowledge that will inform food manufacturers on how to target consumers with high willingness to pay. The sub-objectives are the following: 1) to identify consumer-related factors affecting the acceptance of, and preference for, nutrition-modified and functional dairy foods products (Chapter 2);2) to estimate the implicit price of different types of health claims allowed by Reg. No.1924/2006, conditional on the main factors affecting consumer acceptance of these products, as suggested by the systematic literature review in Chapter 2 (Chapter 3); 3) to investigate the existence of price dispersion due to asymmetric information between manufacturers and consumers that prevents firms to target consumers with a higher value of willingness to pay (Chapter 4); and lastly, 4) to assess the role of consumers' psychological traits, such as the individual level of body image satisfaction, on their decision to select functional products (Chapter 5).

Chapter 2 provides a systematic review of the existing literature on consumer acceptance of, and preference for nutrition-modified and functional dairy products published in peer review journals during the period from 1999 to 2013. The findings of the selected studies confirm those of other literature reviews on how consumers' characteristics and a product's intrinsic attributes influence consumers' acceptance of, and preference for nutrition-modified and functional foods. Chapter 2 results are in line with those of other literature reviews confirming that extrinsic product attributes, such as the type of health benefit (and the claimed effectiveness on human health (disease risk reduction vs. general functional activity) affect consumer acceptance of, and preferences for, products with health claims. The studies reviewed in Chapter 2 present the new insight that functional foods acceptance depends also upon consumers' familiarity with the brand name associated with the health claim. The way brands affect consumers' evaluation of nutrition-modified and functional products has not been highlighted in any prior literature review.

Chapter 3 assesses the implicit price of health claims allowed by the European Regulation No.1924/2006. Using the findings of Chapter 2, the implicit price of each health claim is estimated conditionally on three factors: the type of health benefit provided, the guaranteed level of efficacy on human health, and whether a claim is supported by a familiar brand. The econometric estimates show different values associated with the different claimed benefits. 
The claims with the highest implicit prices are those lowering/managing cholesterol blood level, and those supporting bone health. The results also suggest that a claim's efficacy matters in determining a product's premium price: in the case of cholesterol lowering products, disease risk reduction claims have a much larger implicit price than general function ones. Furthermore, most health claims supported by a familiar brand benefit from a higher implicit price than those supported by less familiar ones. Thus, using actual sales data, the results from Chapter 3 provide empirical support to the findings of the systematic literature review discussed in Chapter 2.

Chapter 4 explores whether manufactures' investments in credence attributes such as health claims, "organic", and "natural" claims, ${ }^{1}$ enable food manufacturers to reach systematically consumers with higher WTP for the attribute bundles they offer. For credence attributes, whose presence cannot be assessed a priori, asymmetric information may result in consumers not being willing to pay a high price for products carrying them, which may impair manufacturers' ability to differentiate their products. The findings of Chapter 4 indicate that manufacturers with a larger portfolio of products carrying credence attributes can systematically reach consumers with a higher WTP for their products. Such result suggests that introducing products with credence attributes, such as health claims, "organic" or "natural" claims in a producer's portfolio, may increase the extent of product differentiation, and result in an improved reputation. Thus, firms which invest strategically in credence features may be able to segment the market more effectively than other firms and reach consumers with a higher WTP.

Chapter 5 explores the relationship between the choice of purchasing functional alternatives, knowledge about leading functional brands, and one's level of satisfaction with his/her own body image. In other words Chapter 5 creates a framework to validate and expand upon the findings of the literature reviewed in Chapter 2, assessing the synergic effect of a psychological trait and consumer brand knowledge on functional alternatives' choice. In fact, the existing literature has not explored yet the effect of body image satisfaction on functional foods consumption, even though other literature has found that being unsatisfied with one's own body image is associated with long-term unhealthy eating behavior, and a lower selection of healthy foods. Chapter 5 tests the hypothesis that the lower the level of own body image satisfaction, the smaller the purchased quantity of functional options is. Furthermore, it measures how functional brand knowledge and reading the nutritional label affects the number of functional alternatives purchased. The findings presented in Chapter 5 indicate that the level of own body image dissatisfaction is negatively correlated with the likelihood of selecting functional options from a virtual supermarket shelf, that the predicted number of functional product purchased increases with the level of body satisfaction, functional brand knowledge, and reading the nutrition label.

Chapter 6 synthetizes the main findings of the thesis, outlines the policy and business implications of the main results, highlights limitations, future research directions, and summarizes the main conclusions. The main conclusions of this thesis are as follows:

1. A systematic review of the literature finds that consumer related characteristics such as age, gender, nutritional knowledge, and exposure (direct or indirect) to chronic diseases, affect positively the acceptance of, and preference for, nutrition-modified and functional dairy products (Chapter 2);

\footnotetext{
${ }^{1}$ Premium price for attributes as "Organic" and "Natural", estimated in Chapter 3, is higher than those of many

"General function" health claims.
} 
2. Psychological factors, such as interest in health and perceived self-efficacy, influence positively the acceptance of, and preference for functional dairy products; instead, food neophobia and body image dissatisfaction reduce consumer's acceptance and preference (Chapter 2 and Chapter 5);

3. The systematic literature review highlights a consensus that claims supported by familiar brands, as well as "risk reduction" (vis-à-vis "general function") ones increase consumers' acceptance of functional dairy products; brand familiarity and a claim's effectiveness, can lead to higher health claims' implicit prices (Chapter 2 and Chapter $3)$;

4. In the Italian yogurt market the implicit prices of "risk reduction" claims is higher than the "general function" ones (respectively, $+222.6 \%$ vs $+85.5 \%$ of the baseline alternative's price in the case of cholesterol reduction health claim), and in three out of four cases, health claims supported by familiar brands have higher implicit price that those supported by less-familiar ones (Chapter 3);

5. Manufacturers operating in the Italian yogurt market investing in credence attributes (health claims, organic and natural) gain the ability to target consumers with higher WTP by including two or more products carrying credence attributes in their portfolio (Chapter 4);

6. Natural and organic claims lead to implicit prices which are (approximately) $+46.6 \%$ and $+102.0 \%$ over the price of the baseline alternative (Chapter 3). Such implicit prices are comparable to those of some health claims (Chapter 3 and Chapter 4);

7. Using a virtual shelf choice setting, of a sample of Italian yogurt consumers, reveals that respondents less satisfied with their own body image select a lower number of functional yogurt packages (Chapter 5);

8. Consumers' functional brand familiarity is positively related to the number of functional alternative selected in a virtual shelf shopping experience (Chapter 5). 


\section{Acknowledgements}

I would never have been able to finish my dissertation without the guidance that I received from many people, the help of many friends, and the support from my family and wife. Thus, I would like to thank all the people who have supported and helped me in different ways throughout the last four years.

I wish to express my deep gratitude to Alfons Oude Lansink and Gianluca Nardone for having given me the opportunity to do the Ph.D. at Wageningen University. Alfons, thanks for having hosted me in the Business Economics group, for the continuous support during last four years, as well as for having been a great example of a group leader. I would like to thank particularly Gianluca for having always invested in me during this educational training that took several years. I feel honoured to have met you both through the duration of my vocational training.

This thesis not has been possible without Alessandro's guidance, a role model in academia as well as a personal supporter during my difficulties. As my supervisor and mentor, he has taught me more than I could ever give him credit for here. He has shown me, by his example, what a good scientist should be. Further, thanks to Rosaria for her nearness along these years, she was always supportive in many important decisions.

I would like to express my gratitude to all the people in the Business Economics Group who provided me a warm, inspiring and functional research environment. I would be remiss if I did not thank Anne and Jeanette, who deserve credit for providing much assistance with administrative tasks, reminding impending deadlines, and keeping the work running smoothly. Also, many people from the Dipartimento di Scienze Agrarie, degli Alimenti e dell'Ambiente of Foggia University deserve my special thanks for the support and encouragement, therefore I would like to thank Marcella, Rosa, Maurizio and Antonio ("Loppy").

Moving on to the friends in Wageningen, I would like to thank the Leeuwenborch's Italians for the many cheerful time together: Valentina, Walter "Jep Gambardella", Niccolò, Luca, Carla, Paola, Raffaele and Lavinia. Also thanks to my friends Franco, Xudong, Rob, Lisa and my great officemate Tariku for the many coffee, lunch, and dinner together as well as for the mutual support over last four years.

Nobody has been more important to me in the pursuit of this Ph.D. than the members of my family. I would like to thank my parents Luisa and Giovanni, as well as my brother, Agostino, whose love and guidance are with me in whatever I pursue. Last but not the least, I wish to thank my loving and supportive wife, Francesca, who provided unending support and patience for all the time I spent far from her. This work is dedicated to you. 


\section{Francesco Bimbo}

Wageningen School of Social Sciences (WASS)

Completed Training and Supervision Plan

\section{\%ั \\ Wageningen School \\ of Social Sciences}

Name of the learning activity

A) Project related competences

BEC-31306 Organisation of the

Agribusiness

AEP-60306 Advanced Econometrics

Summer School "Theory and Practice of Efficiency \& Productivity Measurement: Parametric Efficiency and Productivity Analysis"

B) General research related competences
WASS, Wageningen

2013

WASS PhD day, Wageningen

XIIIIth EAAE - European

Association of Agricultural

Economists Congress, Ljubljana

ICAE International Conference

of Agricultural Economist,

Milano

161th Seminar of European

Association of Agricultural

Economists (EAAE) -

11th International European

Forum (Igls-Forum)

Writing PhD Research Proposal

Business Economics Group Meeting

WUR, Wageningen

2013

WUR, Wageningen
2013

2014

2015

ECTS*

Year

6

6

4

6

20132016

3

C) Career related competences/personal development

Supervision of BSc student

WUR, Wageningen

2015

1

*One credit according to ECTS is on average equivalent to 28 hours of study load 


\section{FUNDING}

The research described in this thesis was financially supported by University of Foggia Dipartimento di Scienze Agrarie, degli Alimenti e dell'Ambiente. 